Application of an Extended Huygens' Principle to Scattering Discontinuities in Waveguide

\title{
RH Geschke
}

Dissertation presented for the Degree of Doctor of Philosophy in Engineering at the University of Stellenbosch

Promotors: Prof DB Davidson, Prof P Meyer and Dr RL Ferrari April 2004 


\section{Declaration}

I, the undersigned, hereby declare that the work contained in this dissertation is my own original work, unless stated otherwise, and it has not previously in its entirety or in part been submitted at any university for a degree. 


\begin{abstract}
The implementation and verification of a recently proposed Huygens' principle in hollow lossless waveguide is described. The extended Huygens' principle is applicable to the scattering effect of arbitrary obstacles in waveguide and allows the coupling of a volume Finite Element discretization with a quasi Method of Moments surface element approach. A review of the technique is given and expressions for the two port scattering parameters are derived. The implementation is restricted to posts in rectangular waveguide, although the technique may also be applied to general scattering problems in waveguide. Finite Elements for the volume of the obstacle are selected and a basis set is proposed. The elemental finite element matrices are derived for a low and higher order basis set, while the validity of the derivations is established by considering a simple waveguide example. A coupled set of matrix equations is constructed that can be solved for the unknown surface electric and magnetic fields and the volume magnetic fields. A set of test examples is chosen to verify the implementation. The convergence behaviour is examined for two test cases. The dissertation concludes with an evaluation of the technique and recommendations for future work.
\end{abstract}




\section{Opsomming}

Die implementasie en verifiëring van 'n onlangs voorgestelde Huygens beginsel in leë golfleier word beskryf. Die uitgebreide beginsel van Huygens is van toepassing op die strooiingseffek van arbitrêre voorwerpe in verlieslose golfleier en beskryf die koppeling tussen ' $n$ volume eindige element diskretisasie met 'n oppervlakelement kwasi-moment metode. 'n Oorsig oor die tegniek word gegee en uitdrukkings vir die tweepoort strooiingsparameters word afgelei. Die implementering word beperk tot penne in reghoekige golfleier, alhoewel die tegniek op algemene golfleier strooiingsprobleme van toepassing is. Eindige elemente word gekies vir die volume van die voorwerp en 'n basisfunksie versameling afgelei van bestaande basisfunksies. Lae en hoë orde element matrikse word afgelei en die geldigheid van die afleidings word nagegaan deur ' $n$ eenvoudige golfleier probleem op te los. 'n Gekoppelde stel matriksvergelykings word opgestel in terme van die onbekende oppervlak elektriese en magnetiese velde. 'n Aantal toetsvoorbeelde word gekies om die implementasie te verifieer. Konvergensiegedrag word ondersoek vir twee toetsgevalle. Die proefskrif sluit af met ' $n$ evaluasie van die tegniek en aanbevelings vir verdere werk. 


\section{Acknowledgements}

I would like to thank the following institutions: the South African National Research Foundation (NRF) for financial support towards my research and the Trinity College visiting Scholars fund whose support made my visit to Dr Ferrari in Cambridge possible.

I gratefully acknowledge the assistance of the following people who helped me while completing this project: my three promotors each of whom provided valuable inputs to the progress of this project; my family for their support and my husband Mark for his support and many cups of coffee. 


\section{List of Abbreviations}

FE Finite Element

FEM Finite Element Method

MM Mode Matching

FE-MM Finite Element Mode Matching

PEC Perfect Electric Conductor

PMC Perfect Magnetic Conductor

CT/LN constant tangential, linear normal

LT/QN linear tangential, quadratric normal 


\section{Contents}

1 Introduction 1

1.1 Discontinuities in Waveguide . . . . . . . . . . . . . 1

1.1.1 Modal Analysis (Mode Matching) . . . . . . . . . . 2

1.1.2 Moment Methods . . . . . . . . . . . . . . . 2

1.1.3 Finite Element Methods (FEM) $\ldots \ldots \ldots \ldots \ldots$

1.1.4 Time Domain Formulations $\ldots \ldots \ldots \ldots$

1.1.5 Hybrid Methods . . . . . . . . . . . . . . 4

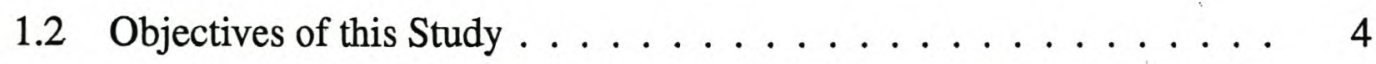

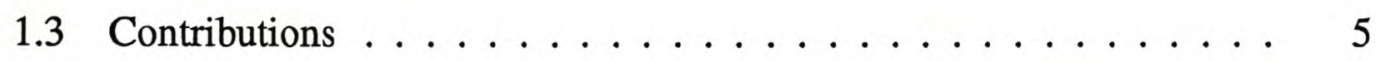

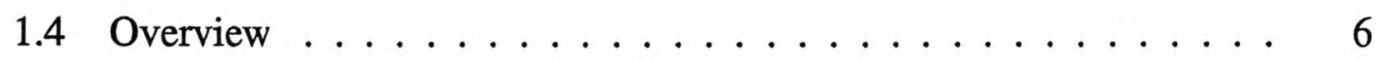

2 The Huygens Formulation $\quad 7$

2.1 Introduction $\ldots \ldots \ldots \ldots \ldots \ldots \ldots \ldots \ldots \ldots \ldots \ldots \ldots \ldots$

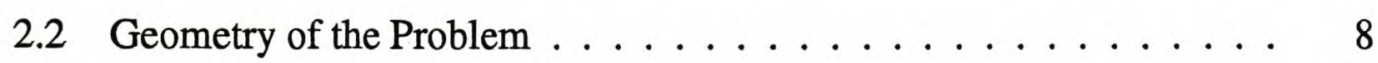

2.3 Hollow Lossless Waveguide Modal Fields . . . . . . . . . . . 8

2.4 The Basis of the Huygens' Principle $\ldots \ldots \ldots \ldots$

2.5 The Waveguide Green Functions . . . . . . . . . . . . 10

2.6 Linking the Surface and Volume of the Scatterer . . . . . . . . 11

2.7 Solving the Volume Magnetic Fields and Surface Tangential Electric

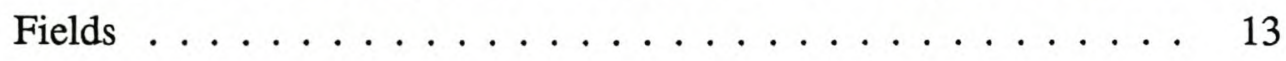

2.8 The Generalized Scattering Matrix $\ldots \ldots \ldots \ldots \ldots \ldots$ 
2.8.1 The Generalized Scattering Parameter $S_{21}^{(m n)} \quad \ldots \ldots \ldots$

2.8.2 The Generalized Scattering Parameter $S_{11}^{(m n)} \ldots \ldots$

2.8.3 The Generalized Scattering Parameter $S_{12}^{(m n)} \ldots \ldots 17$

2.8.4 The Generalized Scattering Parameter $S_{22}^{(m n)} \ldots \ldots 18$

2.9 Conclusion . . . . . . . . . . . . . . . 18

3 Implementation of Posts in Waveguide 19

3.1 Introduction . . . . . . . . . . . . . . 19

3.2 Simplifications ........................ 20

3.3 Finite Element Discretization . . . . . . . . . . . . . . 21

3.3.1 Prismatic elements . . . . . . . . . . . . . 21

3.3.2 The Finite Element Matrices . . . . . . . . . . . . . 25

3.4 Testing of Finite Element Basis Functions . . . . . . . . . . . . 29

3.5 Connection between inner H-fields and tangential E-fields . . . . . 30

3.5.1 Evaluation of $\left[B_{E}\right] \ldots \ldots \ldots \ldots \ldots$

3.6 Testing of Connection between Inner Volume and Outer Surface . . . 32

3.7 The Waveguide Dyadic Green Functions . . . . . . . . . . . . . . . 32

3.8 Integration Scheme $\ldots \ldots \ldots \ldots \ldots \ldots$

3.8 .1 Gaussian Quadrature . . . . . . . . . . . . 34

3.8.2 Coordinate Rotation . . . . . . . . . . . . 34

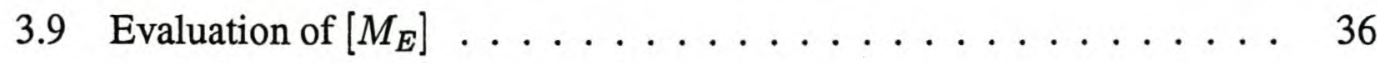

3.10 Evaluation of $\left[M_{H}\right] \ldots \ldots \ldots \ldots \ldots$

3.11 Solution of Matrix Equations . . . . . . . . . . . . . . 42

3.11.1 Perfect Electric Conducting (PEC) Posts . . . . . . . . . . . . 43

3.11.2 Perfect Magnetic Conducting (PMC) Posts . . . . . . . . . 43

3.11 .3 Dielectric Posts . . . . . . . . . . . . . 43

3.12 Conclusion .......................... 44 
4 Results for Posts in Waveguide 4

4.1 Introduction . . . . . . . . . . . . . . 46

4.2 Perfectly Electric Conducting (PEC) Posts . . . . . . . . . . . . . 47

4.3 Perfect Magnetic Conducting (PMC) Posts . . . . . . . . . . . . . . 49

4.4 Dielectric and Magnetic Posts . . . . . . . . . . . . . . 49

4.4.1 Test example 1: A thin Magnetic Post . . . . . . . . . . . 49

4.4.2 Test example 2: A thick Dielectric Post . . . . . . . . 53

4.4.3 Test example 3: A multiple Post Configuration . . . . . . . 53

4.4.4 Test example 4: A lossy Dielectric Post . . . . . . . . . 56

4.5 Convergence Requirements . . . . . . . . . . . . . . . 56

4.6 Summary ............................. 59

5 Conclusions and Recommendations $\quad 61$

5.1 Introduction .................... 61

5.2 Evaluation of the Technique $\ldots \ldots \ldots 6$

5.3 Applications ...................... 62

5.4 Possible Extensions of the Application . . . . . . . . . . . 63

5.4.1 Geometric Extensions ............... 63

5.4 .2 Efficient Matrix Solutions .............. 64

5.5 Conclusion ............................ 64

A Notes for a Huygens' Code Developer I

A.1 Programming Tools ................. I

A.2 Mesh Generation . . . . . . . . . . . . . I I

A.3 Developing Strategy: A Modular Approach . . . . . . . . . . . . III 
B Derivation of Tangential Basis Function Component IV

B.1 $\hat{\mathbf{n}} \times \mathrm{W}_{l} \ldots \ldots \ldots \ldots \ldots \ldots$

B.2 $\hat{\mathbf{n}} \times \mathrm{M}_{l} \ldots \ldots \ldots \ldots \ldots \ldots$

B.3 $\hat{\mathbf{n}} \times \mathbf{F}_{l} \ldots \ldots \ldots \ldots \ldots \ldots$

$\mathrm{B} .4 \hat{\mathbf{n}} \times \mathbf{K}_{l} \ldots \ldots \ldots \ldots \ldots \ldots$

C CT/LN versus LT/QN basis sets $\quad$ VII 


\section{List of Figures}

2.1 A cross section through hollow waveguide with inhomogeneous obstacle included. The $\mathrm{z}$ axis is aligned with the direction of propagation. The surfaces forming part of $\Gamma, \Gamma=\Gamma_{1} \cup \Gamma_{2} \cup \Gamma_{L} \cup \Gamma_{W}$, are indicated. 8

2.2 A schematic diagram representing the scattering coefficients $\mathbf{a}^{(1)}, \mathbf{a}^{(2)}$, $\mathbf{b}^{(1)}$ and $\mathbf{b}^{(2)}$. Note the deviation from the usual definition of $\mathbf{a}$ and $\mathbf{b}$.

3.1 A homogeneous cylindrical post, either dielectric, magnetic or conducting, extending between the broad faces of a rectangular $T E_{10}$ waveguide. The broad wall of the waveguide has dimension $a$, while the narrow wall has the dimension $b . \ldots \ldots \ldots \ldots$

3.2 The prismatic finite element geometry. Side face 1 is defined by nodes 2 and 3. Side face 2 is defined by nodes 3 and 1 , and side face 3 is defined by nodes 1 and 2 . The lengths, $d_{i}$, with $i=1,2,3$, of each side face are indicated. The element extends from $y=0$ to $y=b$, with $b$ the height of the waveguide, as indicated in figure $3.1 \ldots \ldots \ldots$

3.3 Prismatic basis function on a prismatic element face, basis set (3.2) in column 1. Column 2 shows the cross-product of the normal vector $\hat{\mathbf{n}}$ with each basis function that is required for surface integration over the surface patch area. . . . . . . . . . . . . .

3.4 Transverse basis functions on triangular cross-section of prismatic element at $y=b$. The first three are for basis set (3.2), while the full set is required for basis set (3.3). . . . . . . . . . . . 23

3.5 Geometry of a test problem with material discontinuities . . . . . . 29

3.6 The magnetic field $\mathrm{z}$ component at $x=0$, obtained via a finite element solution at $8 \mathrm{GHz}$ with a $\mathrm{CT} / \mathrm{LN}$ basis set. Two mesh sizes were used to demonstrate convergence behaviour. . . . . . . . . . 
3.7 Magnitude of $H_{z}$ field at $\mathrm{x}=0$, using the connection matrix and Finite Element solution, compared to an analytic solution. Two mesh sizes

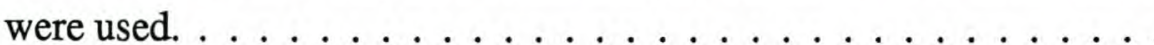

3.8 Top view of two partially $z$ overlapping surface patches, projected onto the $\mathrm{z}$ axis. The integration over $z$ and $z^{\prime}$ is split into three parts, i.e. $\int_{z_{a}}^{z_{1}} \int_{z_{1}}^{z_{2}} \ldots d z d z^{\prime}, \int_{z_{1}}^{z_{b}} \int_{z_{1}}^{z_{b}} \ldots d z d z^{\prime}$ and $\int_{z_{1}}^{z_{b}} \int_{z_{b}}^{z_{2}} \ldots d z d z^{\prime} \ldots \ldots \ldots$

3.9 Top view of a surface patch: definition of uv axis with respect to $\mathrm{xz}$ axis. 35

3.10 Top view of two surface patches: the use of the parameter $s$ for a source patch integrated in a positive z-direction. For this case $s=1$. . . .

3.11 Top view of two surface patches: the use of the parameter $s$ for source patch integrated in a negative z-direction. For this case $s=-1$. . . .

3.12 Position of possible entries of the non-finite element matrices of equations (3.60) and (3.61). $N$ is the number of total degrees of freedom and includes both magnetic and electric coefficients, $M$ is the number of electric field unknowns and $K$ is the number of surface tangential magnetic field unknowns. The second row shows these matrices in compressed format (involving only surface coefficients) as used in equation (3.60). $K=M$ when the lowest order basis set is used. . . .

4.1 two posts, no symmetry . . . . . . . . . . . . . . 47

4.2 large centre post, symmetric . . . . . . . . . . . . . 47

4.3 off-centre thin post $\ldots \ldots \ldots$. . . . . . . . . . 47

4.4 two posts in non symmetric configuration . . . . . . . . . . . 47

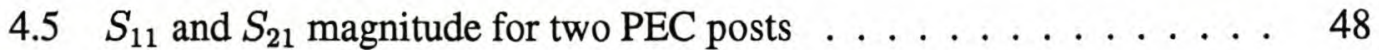

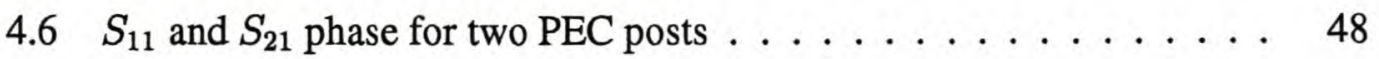

$4.7 S_{11}$ and $S_{21}$ magnitude for a PMC post . . . . . . . . . . 50

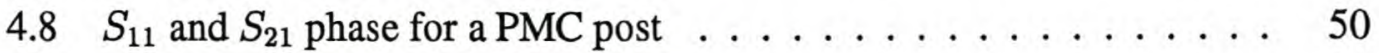

$4.9 S_{11}$ and $S_{21}$ magnitude for a thin magnetic post $\ldots \ldots \ldots 51$

$4.10 S_{11}$ and $S_{21}$ phase for a thin magnetic post . . . . . . . . . 51

$4.11 S_{11}$ and $S_{21}$ magnitude for a thick dielectric post . . . . . . . . . 52

$4.12 S_{11}$ and $S_{21}$ phase for a thick dielectric post . . . . . . . . 52 
$4.13 S_{11}$ and $S_{21}$ magnitude for two posts $\ldots \ldots \ldots \ldots \ldots$

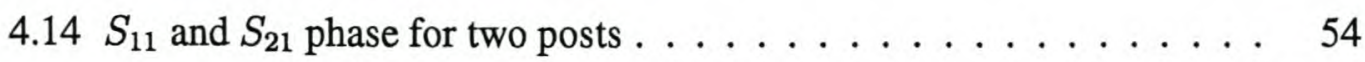

$4.15 S_{11}$ and $S_{21}$ magnitude for a lossy post $\ldots \ldots \ldots \ldots \ldots$

$4.16 S_{11}$ and $S_{21}$ phase for a lossy post $\ldots \ldots \ldots \ldots \ldots$

$4.17 S_{11}$ and $S_{21}$ power sum for a lossy post $\ldots \ldots \ldots \ldots \ldots$

$4.18 S_{11}$ error relative to CST Microwave Studio ${ }^{\circledR}$ results for an $\frac{a}{2}$ width centre post. The variables are the number of even waveguide TE modes considered and the number of volume elements. 8 integration points per element were used. . . . . . . . . . . . . 57

$4.19 S_{11}$ error relative to CST Microwave Studio ${ }^{\circledR}$ results against number of even waveguide modes. . . . . . . . . . . . 57

4.20 $S_{11}$ error relative to CST Microwave Studio ${ }^{\circledR}$ results for an $\frac{a}{5}$ width centre post. The variables are the number of even waveguide TE modes considered and the number of volume elements. 8 integration points

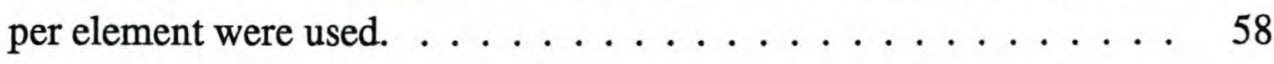

$4.21 S_{11}$ error relative to CST Microwave Studio ${ }^{\circledR}$ results against number

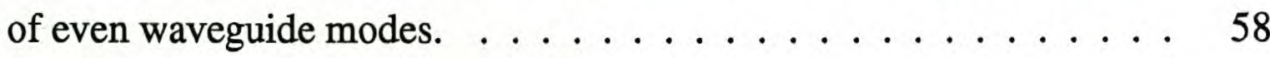

A.1 3D homogeneous multi-layer prismatic mesh constructed from a 2D triangular mesh $\ldots \ldots \ldots \ldots \ldots \ldots$ II

B.1 The two non-zero simplex coordinates for a patch element. . . . . . IV

C.1 Geometry of a test problem with material discontinuities, repeated here

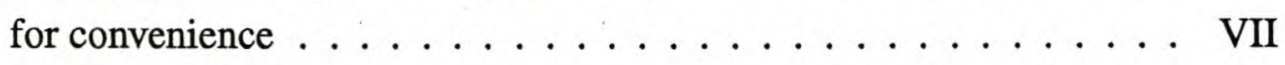

C.2 Magnitude of $H_{z}$ field at $\mathrm{x}=0$, using the connection matrix and Finite Element solution, compared to an analytic solution. The waveguide was discretized into 170 elements for both the CT/LN and LT/QN case. VIII 


\section{Chapter 1}

\section{Introduction}

During World War II research on radar and microwaves received top priority, drawing physicists and engineers to work in this field. In this time Schwinger and Marcuwitz considered discontinuities in waveguide for radar applications, including posts in waveguide for filter applications [1]. At the time they were limited to approximate methods not requiring substantial computing power to solve these problems. Some of these results were later extended and included in the Waveguide Handbook [2]. After the end of the war, a paper [3] appeared on the rigorous treatment of discontinuities in waveguides. In this paper, integral expressions for the electric and magnetic fields in waveguide in terms of integrals of magnetic and electric surface currents on the obstacles, appear. A sequel to this paper was promised but never appeared, since after the end of the war the authors' priorities changed. A set of lecture notes by Julian Schwinger presented to his collegues at the MIT Radiation Laboratory is available [4]. At the present time various numerical techniques are applied to waveguide discontinuity problems. Some of the techniques used are moment methods, modal analysis and finite element methods, which are all frequency domain techniques. A time domain technique often used for waveguide scattering problems is the finite difference time domain method (FDTD).

\subsection{Discontinuities in Waveguide}

Typical applications of discontinuity solvers in waveguide are the analysis and design of filters for radar and the satellite industry [5] and beam forming networks for antennas [6], to name only a few. A few examples of the large variety of waveguide discontinuities routinely considered are waveguide transitions, step discontinuities, bifurcations, Tjunctions, aperture couplers, phase shifters, mode polarizers and dielectric resonator 
filters. Many waveguide problems are solved effectively by the established methods considered below. Some of these geometries, especially those involving dielectric or magnetic materials, can benefit from more effective techniques than those available at present.

\subsubsection{Modal Analysis (Mode Matching)}

Characterizing abrupt discontinuities by summing all the possible waveguide modes at both sides of the plane of the discontinuity and enforcing continuity at this plane, is the basis of the mode matching technique. A system of equations results, relating the amplitudes of all the propagating and non-propagating modes through the generalized scattering parameters.

Wexler [7] considered the analysis of various types of discontinuities, such as irises, multiple junctions and interfaces between waveguides of varying cross-sections, using modal analysis.

\subsubsection{Moment Methods}

Moment methods involve the solution of an integral equation over volumes or surfaces subdivided into elements. Around the same time that modal analysis became a popular tool, moment methods were employed for successful solutions of scattering wire objects such as wire antennas in free space $[8,9]$. Solutions to scattering in waveguides for coupling apertures [10], conducting [11] and dielectric posts [12,13] followed. The waveguide solution requires knowledge of the waveguide Green functions, which are very slowly converging double infinite series [14]. A three dimensional volume moment method implementation for arbitrary shaped dielectric bodies in rectangular waveguide appeared in [15]. Multiple dielectric posts in waveguide are considered in [16]. The analysis of waveguide junctions (T-junctions and waveguide bends) is performed with a boundary element method in [17]. Another boundary element implementation is found in [18] where results for metallic and dielectric posts are presented.

The disadvantage of these methods is the double volume integrations required over source and observation elements in the general case of dielectric materials. The Green function convergence may require special acceleration techniques, which are dependent on the exact form of the Green and weighting functions used [15]. The solutions of conducting obstructions require only surface integrals and are therefore solved more effectively. 


\subsubsection{Finite Element Methods (FEM)}

Since the vector based finite element basis functions were suggested $[19,20]$ as an alternative to nodal based scalar basis functions [21,22], finite element solutions were no longer contaminated by spurious solutions associated with the less sophisticated nodal elements [23, 24]. The finite element method became a viable solution for waveguide discontinuity solutions. In [25] the three- dimensional FE analysis of a waveguide discontinuity is described. The waveguide with discontinuity is terminated on both sides by planes suitably far removed from the discontinuity to ensure that evanescent modes have decayed. Boundary conditions on these planes, that include the dominant mode excitation, are derived. Another approach appears in [26], where two types of basis functions are implemented and compared. The 3D FE solution process may be extended to obtain the generalized (multi-mode) scattering matrix [27]. The disadvantage is that often a large portion of the empty waveguide must be discretized along with the scattering obstacle. In terms of both degrees of freedom and computational time required, this approach is often not optimal. The numerical finite element code ANSYS ${ }^{\circledR}$ is commercial software widely used for finite element solutions and will also be used to verify results obtained.

\subsubsection{Time Domain Formulations}

The finite difference time domain method (FDTD) uses a pulse input to analyze a device or structure and deduces frequency domain response by applying a digital Fourier transform to the time domain data it obtains. The technique is versatile; the main limitation of the basic formulation being the discretization which is often in terms of rectangular blocks, influenced partly by the smallest geometrical elements to be modelled. Radiating or absorbing boundary conditions are implemented for open boundaries. An FDTD implementation for posts in waveguide is found in [28].

An advantage of the FDTD technique is that frequency data over a range of frequencies may be obtained from a single pulse excitation. All other frequency domain methods mentioned in the previous sections require application of the technique at every frequency point of interest, unless adaptive sampling and interpolation models are used to interpolate frequency data determined at a few well-chosen frequency points [29]. A commercial time domain code, CST Microwave Studio ${ }^{\circledR}$, has been used to obtain S-parameter data for comparative purposes in chapter 4 . 


\subsubsection{Hybrid Methods}

Hybrid methods usually combine two techniques to realize the advantages of both and ideally, provide an efficient method of solving the problem under consideration.

\section{Finite Element- Mode Matching Methods}

The combination of finite element and mode matching techniques (FE-MM) [30] to solve waveguide scattering problems is very popular. The finite element method and mode matching are very effective together for the scatterer in waveguide problems. Since any arbitrary scatterer can be discretized using finite elements and then linked with a waveguide modal expansion at an interface, the discretized region is much smaller than if only finite elements were used. Implementations of these appear in [31] and [32]. A disadvantage of the technique is that empty regions around the scatterer are still discretized into finite elements.

\section{An Extended Huygens Principle}

Recently a new technique was proposed [33] for the solution of waveguide scattering problems. This is a hybrid technique that includes a region discretized into finite elements and a moment method type double integration over the surface of the object. The tangential electric and magnetic fields link the inner and outer region. The volume meshed into finite elements is only the scatterer itself, while the boundary between the scatterer and the waveguide region is discretized into surface elements. This is potentially more computationally efficient than the FE - MM method.

\subsection{Objectives of this Study}

Since the extended Huygens' principle has not been implemented to date, the purpose of the study was to implement and verify this suggested technique. The verification includes surmounting the challenges presented by an implementation. A very important part of the verification is the investigation into the validity and practicality of this technique through results obtained. Also, as a first implementation, an idea of the strengths and weaknesses of the technique should be formed. Guidelines for the appropriate use of the technique are required. Questions to be answered are how fine the finite element discretization should be, how many modes are required to be summed and how many integration points are required for converged results. 


\subsection{Contributions}

The main contribution of this thesis is the first implementation of this hybrid technique specifically for a simplified $T E_{p 0}$ symmetry. The detailed contributions may be summarized as:

(a) Implementation of a numerical integration scheme to avoid the analytic integration of matrix entries and the singular term in the electric field Green function

The numerical scheme allows flexibility in the choice of a basis set, as basis set upgrades do not require changing the surface integral routines. The finite element basis sets are hierarchal, which means that to upgrade to a higher order set requires additional basis functions to the existing ones. This translates into an extension of the elemental matrices without any changes to the lower order functions already implemented.

(b) Adaptation of two existing basis sets to form a $3 D$ linear tangential quadratic normal (LT/QN) prismatic basis set

(c) Derivation of elemental $S$ and $T$ matrices for the two basis sets

(d) Derivation of certain elemental B matrix entries for the constant tangential linear normal $(C T / L N)$ and linear tangential quadratic normal (LT/QN) basis set for prismatic elements

This involves the connection between the fields of the volume of the scatterer and the surface fields.

(e) A test to determine the validity of the connection between the inner volume and the surface of the scatterer

This test is primarily of use as a debugging aid for the developer implementing upgrade basis function sets. It also provides a visual indication of the modelling abilities of various basis sets.

(f) Results for homogeneous dielectric posts in waveguide

These are the first results obtained with the use of the Huygens formulation, using CT/LN elements. This is significant, as it is the first verification of the suggested formulation.

(g) Insertion of a missing factor where the source and observation point is found on the same boundary surface

This factor was not included in the original paper [33]. 


\subsection{Overview}

In the next chapters we proceed to a description of the technique, details of the implementation and finally the analysis of the results obtained.

The scene is set in chapter 2, where the Huygens formulation for scattering objects or loads in waveguide is described. This details all theoretical aspects of the new technique and is in part a summary and in part a discussion of the material found in [33]. The theory is general and is not limited to a specific scatterer or waveguide geometry. Chapter 3 proceeds to the implementational details for perfectly electric conducting, perfectly magnetic conducting, dielectric and magnetic posts in X-band waveguide, which will serve as test cases for the technique. The implementation has two parts, a finite element section and moment method type surface integrations which are linked by the surface tangential fields. Two finite element basis sets are introduced and the finite element matrix entries are calculated. These derivations are confirmed by a simple finite element test using a partly empty section of X-band waveguide loaded with a lossy material. Having established confidence in the suitability of the basis set, we proceed to connect the inner volume of the scatterer to the empty waveguide through the surface fields, using a connection matrix. The surface integrals required are evaluated using a numerical integration scheme, allowing flexibility in the choice of basis sets.

Chapter 4 presents S-parameter and convergence results for conducting and dielectric posts in X-band waveguides. It is shown that the technique provides excellent magnitude and phase results for a number of representative test problems.

In chapter 5 the technique and results are evaluated and possible further extensions to this work, which may form the basis of future projects, are described. 


\section{Chapter 2}

\section{The Huygens Formulation}

\subsection{Introduction}

Often waveguide scattering problems require the determination of the scattering matrix for a hollow waveguide with an obstruction or load included, such as posts which may be dielectric or metallic, as used in filter applications.

Usually the section of waveguide filled by the scatterer is quite small compared with the rest of the surrounding empty waveguide. Discretization of the entire waveguide by the finite element method (FEM) allows a very general object with varying material properties to be considered with ease, but will require the discretization of a large portion of empty waveguide along with the scattering object. This usually is not computationally efficient.

The Huygens' principle [33] described in this chapter provides a solution to the problem of wasted degrees of freedom. This chapter details how the finite element discretization may be confined to the interior volume and the exterior surface of the volume. The formulation then provides a link between the inner fields of the scatterer and the external waveguide fields through the tangential fields on the surface of the object.

Once the total field, the sum of the incident field and the scattered field due to the scatterer, is known, it is possible to determine the generalized scattering matrix of the waveguide with the scatterer included. The formulation is applicable to general lossless waveguides, although all examples will relate to rectangular waveguide. As excitation the dominant $T E_{10}$ mode is used, although any higher order mode excitation may be considered if required. 


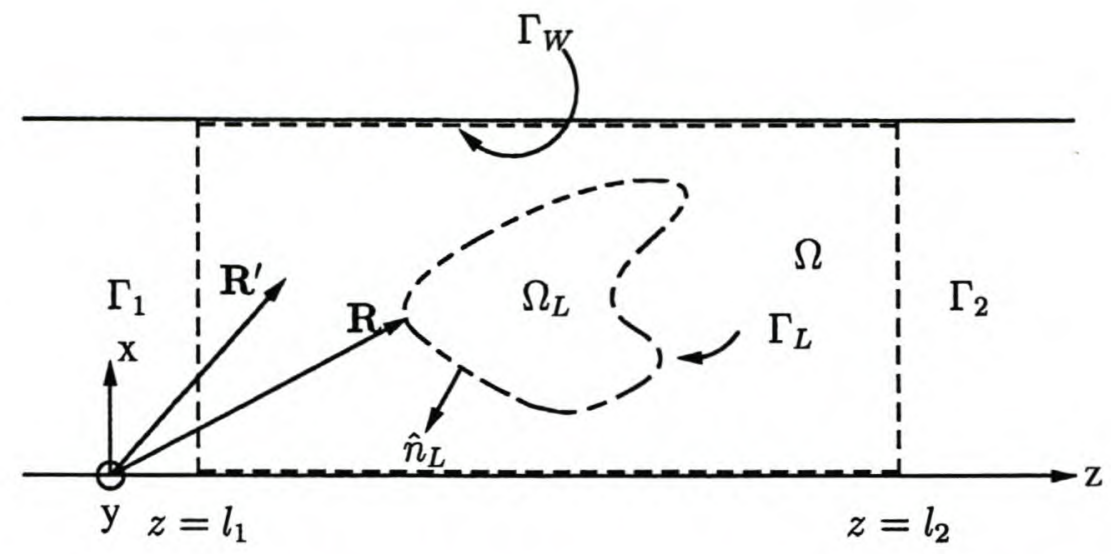

Figure 2.1: A cross section through hollow waveguide with inhomogeneous obstacle included. The $z$ axis is aligned with the direction of propagation. The surfaces forming part of $\Gamma, \Gamma=\Gamma_{1} \cup \Gamma_{2} \cup \Gamma_{L} \cup \Gamma_{W}$, are indicated.

\subsection{Geometry of the Problem}

The typical problem to be solved is the scattering effect of an object, dielectric or perfectly conducting or a combination of both, in an empty hollow waveguide (figure 2.1). The surface of the scatterer is labeled $\Gamma_{L}$, while the region of the scatterer is enclosed in an imaginary rectangular region $\Omega$, that is bounded by the surface $\Gamma$, consisting of $\Gamma_{L}$, the surface $\Gamma_{1}$ to the left and $\Gamma_{2}$ to the right of the object and the surfaces $\Gamma_{W}$ between the transverse planes $\Gamma_{1}$ and $\Gamma_{2}$. The normal vector to the surface $\Gamma_{L}$ is labeled $\hat{\mathbf{n}}_{L}$. The coordinate system is indicated in figure 2.1.

\subsection{Hollow Lossless Waveguide Modal Fields}

The fields inside the empty waveguide region are written as sums of waveguide modes, both TE and TM [33]. Suppressing an $e^{j \omega t}$ phase factor,

$$
\begin{aligned}
& \mathbf{E}^{(i)}(\mathbf{R})=\sum_{m=1}^{\infty} \sqrt{Z_{m}}\left\{a_{m}^{(i)} e^{-\gamma_{m} z}\left[\mathbf{e}_{t}^{m}(\mathbf{r})+\mathbf{e}_{z}^{m}(\mathbf{r})\right]+b_{m}^{(i)} e^{\gamma_{m} z}\left[\mathbf{e}_{t}^{m}(\mathbf{r})-\mathbf{e}_{z}^{m}(\mathbf{r})\right]\right\} \\
& \mathbf{H}^{(i)}(\mathbf{R})=\sum_{m=1}^{\infty} \sqrt{Y_{m}}\left\{a_{m}^{(i)} e^{-\gamma_{m} z}\left[\mathbf{h}_{t}^{m}(\mathbf{r})+\mathbf{h}_{z}^{m}(\mathbf{r})\right]-b_{m}^{(i)} e^{\gamma_{m} z}\left[\mathbf{h}_{t}^{m}(\mathbf{r})-\mathbf{h}_{z}^{m}(\mathbf{r})\right]\right\} \\
& \mathbf{e}_{z}^{m}=\left\{\begin{array}{cc}
0 & \text { TE modes } \\
\frac{1}{\gamma_{m}} \nabla_{t} \times \mathbf{h}_{t}^{m} & \text { TM modes }
\end{array}\right.
\end{aligned}
$$




$$
\mathbf{h}_{z}^{m}=\left\{\begin{array}{cc}
-\frac{1}{\gamma_{m}} \nabla_{t} \times \mathbf{e}_{t}^{m} & \text { TE modes } \\
0 & \text { TM modes }
\end{array}\right.
$$

where $i=1$ corresponds to a point $\mathbf{R}$ to the left or on $z=l_{1}$ and $i=2$ corresponds to a point $\mathbf{R}$ to the right or on $z=l_{2}$.

The waveguide eigenmodes referred to are $T E_{p q}$ and $T M_{p q}$ modes [34]. In [33] an index $m$ is associated with every distinct $T E_{p q}$ and $T M_{p q}$ mode.

With $m$ and $n$ any two mode indices, the transverse components of the lossless waveguide modes are normalized to

$$
\int_{0}^{b} \int_{0}^{a} \mathbf{e}_{t}^{(m 0)} \cdot \mathbf{e}_{t}^{(n 0)} d x d y=\delta_{m n}
$$

and

$$
\int_{0}^{b} \int_{0}^{a} \mathbf{h}_{t}^{(m 0)} \cdot \mathbf{h}_{t}^{(n 0)} d x d y=\delta_{m n}
$$

with

$$
\delta_{m n}= \begin{cases}1 & m=n \\ 0 & m \neq n\end{cases}
$$

$\left(\delta_{m n}\right.$, the Kronecker delta, not to be confused with the Dirac delta function appearing in (2.11)).

The waveguide modal functions that are considered here are $T E_{p 0}$ modes only, a result of the simplified geometry of loads that will be introduced in chapter 3 . For the geometries introduced there, the index $m$ may be replaced directly with the index $p$.

\subsection{The Basis of the Huygens' Principle}

An expression for the total electric field inside a waveguide with scatterer

$$
\begin{array}{r}
\mathbf{E}\left(\mathbf{R}^{\prime}\right)=\sum_{m=1}^{M} T \sqrt{Z_{m}} a_{m}^{(1)}\left[\mathbf{e}_{t}^{m}\left(\mathbf{r}^{\prime}\right)+\mathbf{e}_{z}^{m}\left(\mathbf{r}^{\prime}\right)\right] e^{-\gamma_{m} z^{\prime}} \\
+\sum_{m=1}^{N} T \sqrt{Z_{m}} b_{m}^{(2)}\left[\mathbf{e}_{t}^{m}\left(\mathbf{r}^{\prime}\right)-\mathbf{e}_{z}^{m}\left(\mathbf{r}^{\prime}\right)\right] e^{\gamma_{m} z^{\prime}} \\
+T \oint_{\Gamma_{L}}\left\{-j k_{0} Z_{0}\left[\hat{\mathbf{n}}_{L} \times \mathbf{H}(\mathbf{R})\right] \cdot \mathbb{G}_{E}\left(\mathbf{R}, \mathbf{R}^{\prime}\right)+\left[\hat{\mathbf{n}}_{L} \times \mathbf{E}(\mathbf{R})\right] \cdot \mathbb{G}_{H}\left(\mathbf{R}, \mathbf{R}^{\prime}\right)\right\} d \Gamma_{L}
\end{array}
$$


is derived in [33]. It is suggested that the factor

$$
T=\left\{\begin{array}{cc}
2 & \mathbf{R}^{\prime} \text { and } \mathbf{R} \text { both on } \Gamma_{L} \\
1 & \mathbf{R}^{\prime} \text { not on } \Gamma_{L}
\end{array}\right.
$$

is inserted into (2.8) as indicated. The $T$ is similar to the factor found in the EFIE as described by [9] and the Stratton-Chu equation in [35] and is supported by [36], where the similarity between a free space Green function singularity and any waveguide green function singularity is described.

The expression for the total electric field represents the sum of the $M$ incident waveguide modes from the left, with modal amplitudes $a_{m}^{(1)}, N$ incident waveguide modes from the right, with modal amplitudes $b_{m}^{(2)}$ and the scattered field due to the tangential electric $\hat{\mathbf{n}}_{L} \times \mathbf{E}(\mathbf{R})$ and tangential magnetic $\hat{\mathbf{n}}_{L} \times \mathbf{H}(\mathbf{R})$ fields on the surface of the scattering object or discontinuity. Each modal field distribution is separated into a transverse and a propagation directed part, i.e. $\mathbf{E}(\mathbf{R})=\left[\mathbf{e}_{t}^{m}(\mathbf{r}) \pm \mathbf{e}_{z}^{m}(\mathbf{r})\right] e^{\mp \gamma_{m} z}$, with the top sign for positive traveling waves and the bottom sign for negative traveling waves. The observation point $\mathbf{R}^{\prime}$ may be placed anywhere inside the empty section of waveguide, including on the scatterer surface. The source point $\mathbf{R}$ is placed on the outer surface of the scatterer, $\Gamma_{L}$, and integrated over this surface.

$\mathbb{G}_{E}\left(\mathbf{R}, \mathbf{R}^{\prime}\right)$ and $\mathbb{G}_{H}\left(\mathbf{R}, \mathbf{R}^{\prime}\right)$ are the dyadic electric and magnetic Green functions for the empty waveguide. These are double infinite sums of the waveguide modal fields, in general both $T E_{m n}$ and $T M_{m n}$ modes.

For single unit n-th mode excitation from the left (+) or right(-), (2.8) reduces to

$$
\begin{aligned}
\mathbf{E}^{ \pm n}\left(\mathbf{R}^{\prime}\right) & =T \sqrt{Z_{n}}\left[\mathbf{e}_{t}^{n}\left(\mathbf{r}^{\prime}\right) \pm \mathbf{e}_{z}^{n}\left(\mathbf{r}^{\prime}\right)\right] e^{\mp \gamma_{n} z^{\prime}} \\
& +T \oint_{\Gamma_{L}}\left\{-j k_{0} Z_{0}\left[\hat{\mathbf{n}}_{L} \times \mathbf{H}(\mathbf{R})\right] \cdot \mathbb{G}_{E}\left(\mathbf{R}, \mathbf{R}^{\prime}\right)+\left[\hat{\mathbf{n}}_{L} \times \mathbf{E}(\mathbf{R})\right] \cdot \mathbb{G}_{H}\left(\mathbf{R}, \mathbf{R}^{\prime}\right)\right\} d \Gamma_{L}
\end{aligned}
$$

For (2.10) to be useful, the surface tangential electric and magnetic fields must be determined. The process of finding these fields is described in the remainder of this chapter.

\subsection{The Waveguide Green Functions}

The dyadic waveguide Green functions below are those given in [33]. These are the Green functions appearing in [37], adapted for the normalization of waveguide modal 
functions adopted here in (2.5) and (2.6). Expressions for waveguide Green functions are also found in [38]. The waveguide Green functions are

$$
\begin{aligned}
\mathbb{G}_{E}^{ \pm}\left(\mathbf{R}, \mathbf{R}^{\prime}\right) & =\frac{1}{j k_{0} Z_{0}} \sum_{p=1}^{\infty} \frac{Z_{p}}{2}\left[\begin{array}{ccc}
e_{x}^{p}(\mathbf{r}) e_{x}^{p}\left(\mathbf{r}^{\prime}\right) & e_{x}^{p}(\mathbf{r}) e_{y}^{p}\left(\mathbf{r}^{\prime}\right) & \mp e_{x}^{p}(\mathbf{r}) e_{z}^{p}\left(\mathbf{r}^{\prime}\right) \\
e_{y}^{p}(\mathbf{r}) e_{x}^{p}\left(\mathbf{r}^{\prime}\right) & e_{y}^{p}(\mathbf{r}) e_{y}^{p}\left(\mathbf{r}^{\prime}\right) & \pm e_{y}^{p}(\mathbf{r}) e_{z}^{p}\left(\mathbf{r}^{\prime}\right) \\
\pm e_{z}^{p}(\mathbf{r}) e_{x}^{p}\left(\mathbf{r}^{\prime}\right) & \pm e_{z}^{p}(\mathbf{r}) e_{y}^{p}\left(\mathbf{r}^{\prime}\right) & -e_{z}^{p}(\mathbf{r}) e_{z}^{p}\left(\mathbf{r}^{\prime}\right)
\end{array}\right] e^{\mp \gamma_{p}\left(z-z^{\prime}\right)} \\
& -\frac{1}{k_{0}^{2}}\left[\begin{array}{ccc}
0 & 0 & 0 \\
0 & 0 & 0 \\
0 & 0 & \delta\left(\mathbf{R}-\mathbf{R}^{\prime}\right)
\end{array}\right]
\end{aligned}
$$

and

$$
\mathbb{G}_{H}^{ \pm}\left(\mathbf{R}, \mathbf{R}^{\prime}\right)=\frac{1}{2} \sum_{p=1}^{\infty}\left[\begin{array}{ccc}
\mp h_{x}^{p}(\mathbf{r}) e_{x}^{p}\left(\mathbf{r}^{\prime}\right) & \mp h_{x}^{p}(\mathbf{r}) e_{y}^{p}\left(\mathbf{r}^{\prime}\right) & h_{x}^{p}(\mathbf{r}) e_{z}^{p}\left(\mathbf{r}^{\prime}\right) \\
\mp h_{y}^{p}(\mathbf{r}) e_{x}^{p}\left(\mathbf{r}^{\prime}\right) & \mp h_{y}^{p}(\mathbf{r}) e_{y}^{p}\left(\mathbf{r}^{\prime}\right) & h_{y}^{p}(\mathbf{r}) e_{z}^{p}\left(\mathbf{r}^{\prime}\right) \\
-h_{z}^{p}(\mathbf{r}) e_{x}^{p}\left(\mathbf{r}^{\prime}\right) & -h_{z}^{p}(\mathbf{r}) e_{y}^{p}\left(\mathbf{r}^{\prime}\right) & 0
\end{array}\right] e^{\mp \gamma_{p}\left(z-z^{\prime}\right)}
$$

with the upper sign corresponding to $z>z^{\prime}$ and the lower sign corresponding to $z<z^{\prime}$. The function $\delta\left(\mathbf{R}-\mathbf{R}^{\prime}\right)$ is a three dimensional impulse function, written in Cartesian coordinates as $\delta\left(\mathbf{R}-\mathbf{R}^{\prime}\right)=\delta\left(x-x^{\prime}\right) \delta\left(y-y^{\prime}\right) \delta\left(z-z^{\prime}\right)$.

\subsection{Linking the Surface and Volume of the Scatterer}

In (2.10) an expression is given for the total electric waveguide field in terms of the total incident and scattered field due to an obstruction. Tangential electric and magnetic fields on the surface of a scatterer act as the sources for the scattered electric field at any point in the waveguide outside the scatterer surface. It is now required to link the internal scatterer magnetic fields to the external electric field through the surface fields on the scatterer surface. The magnetic fields inside and on the scatterer, of which the surface magnetic fields are a subset, are represented with finite elements. The volume of the scatterer is discretized into any suitable three dimensional $\mathrm{FE}$ discretization that conforms to surface patch (rectangular or triangular) elements on the surface of the scatterer. Let $\bar{\tau}_{i}$ be a basis function in the tangentially continuous vector basis set defined over an element.

Starting from the vector wave equation

$$
\nabla \times \frac{1}{\epsilon_{r}} \nabla \times \mathbf{H}-\mu_{r} k_{0}^{2} \mathbf{H}=0
$$

and applying a weighted residual procedure, we require

$$
\int_{\Omega_{L}} \mathbf{W} \cdot\left\{\nabla \times \frac{1}{\epsilon_{r}} \nabla \times \mathbf{H}-\mu_{r} k_{0}^{2} \mathbf{H}\right\} d \Omega=0
$$


where the test functions $\mathbf{W}$ are chosen from the same set as the basis expansion functions to be employed for $\mathbf{H}$. The first vector Green theorem allows (2.14) to be written as

$$
\int_{\Omega_{L}} \frac{1}{\epsilon_{r}}(\nabla \times \mathbf{W}) \cdot(\nabla \times \mathbf{H})-\mu_{r} k_{0}^{2} \mathbf{W} \cdot \mathbf{H} d \Omega=\oint_{\Gamma_{L}} \frac{1}{\epsilon_{r}}(\mathbf{W} \times \nabla \times \mathbf{H}) \cdot \hat{\mathbf{n}}_{L} d \Gamma
$$

The normal vector $\hat{\mathbf{n}}$, that is pointing outwards from the surface $\Gamma_{L}$, is the same as the normal vector $\hat{\mathbf{n}}_{L}$ employed previously in (2.8) and (2.10). Finally, after more vector algebra, one now finds

$$
\int_{\Omega_{L}} \frac{1}{\epsilon_{r}}(\nabla \times \mathbf{W}) \cdot(\nabla \times \mathbf{H})-\mu_{\tau} k_{0}^{2} \mathbf{W} \cdot \mathbf{H} d \Omega=j k_{0} Y_{0} \oint_{\Gamma_{L}}\left(\hat{\mathbf{n}}_{L} \times \mathbf{W}\right) \cdot \mathbf{E} d \Gamma
$$

Finite element discretization of the magnetic field and the tangential electric field on the surface results in

$$
\begin{aligned}
& \sum_{j} \mathcal{H}_{j}^{ \pm n} \int_{\Omega_{L}} \frac{1}{\epsilon_{r}}\left(\nabla \times \bar{\tau}_{i}\right) \cdot\left(\nabla \times \bar{\tau}_{j}\right)-\mu_{r} k_{0}^{2} \bar{\tau}_{i} \cdot \bar{\tau}_{j} d \Omega \\
& =j k_{0} Y_{0} \sum_{j_{\Gamma_{L}}} \mathcal{E}_{j}^{ \pm n} \oint_{\Gamma_{L}} \hat{\mathbf{n}}_{L} \times \bar{\tau}_{i} \cdot \bar{\tau}_{j} d \Gamma i=1,2, \ldots M_{L}
\end{aligned}
$$

which can be written as the matrix equation

$$
\left([S]-k_{0}^{2}[T]\right)\left\{\mathcal{H}^{ \pm n}\right\}=j k_{0} Y_{0}\left[B_{E}\right]\left\{\mathcal{E}_{\Gamma_{L}}^{ \pm n}\right\}
$$

Considering the last term in equation (2.16), the expression for the $\mathrm{n}$-th mode unit excitation electric field at an observation point $\mathbf{R}^{\prime},(2.10)$, can be used to obtain

$$
\begin{array}{r}
\oint_{\Gamma_{L}}\left(\hat{\mathbf{n}}_{L}\left(\mathbf{R}^{\prime}\right) \times \bar{\tau}_{i}\left(\mathbf{R}^{\prime}\right)\right) \cdot \mathbf{E}^{ \pm n}\left(\mathbf{R}^{\prime}\right) d \Gamma_{L}^{\prime} \\
=2 \sqrt{Z_{n}} \oint_{\Gamma_{L}}\left(\hat{\mathbf{n}}_{L}\left(\mathbf{R}^{\prime}\right) \times \bar{\tau}_{i}\left(\mathbf{R}^{\prime}\right)\right) \cdot\left[\mathbf{e}_{t}^{n}\left(\mathbf{r}^{\prime}\right) \pm \mathbf{e}_{z}^{n}\left(\mathbf{r}^{\prime}\right)\right] e^{\mp \gamma_{n} z^{\prime}} d \Gamma_{L}^{\prime} \\
-2 j k_{0} Z_{0} \sum_{j_{\Gamma_{L}}} \mathcal{H}_{j}^{ \pm n} \oint_{\Gamma_{L}} \oint_{\Gamma_{L}}\left(\hat{\mathbf{n}}_{L}(\mathbf{R}) \times \bar{\tau}_{j}(\mathbf{R})\right) \cdot \mathbb{G}_{E}\left(\mathbf{R}, \mathbf{R}^{\prime}\right) \cdot\left(\hat{\mathbf{n}}_{L}\left(\mathbf{R}^{\prime}\right) \times \bar{\tau}_{i}\left(\mathbf{R}^{\prime}\right)\right) d \Gamma_{L} d \Gamma_{L}^{\prime} \\
+2 \sum_{\Gamma_{\Gamma_{L}}} \mathcal{E}_{j}^{ \pm n} \oint_{\Gamma_{L}} \oint_{\Gamma_{L}}\left(\hat{\mathbf{n}}_{L}(\mathbf{R}) \times \bar{\tau}_{j}(\mathbf{R})\right) \cdot \mathbb{G}_{H}\left(\mathbf{R}, \mathbf{R}^{\prime}\right) \cdot\left(\hat{\mathbf{n}}_{L}\left(\mathbf{R}^{\prime}\right) \times \bar{\tau}_{i}\left(\mathbf{R}^{\prime}\right)\right) d \Gamma_{L} d \Gamma_{L}^{\prime}
\end{array}
$$


Noting that each surface basis function is shared by at most two elements and is zero elsewhere, (2.19) is the sum of the contributions from each surface patch:

$$
\begin{array}{r}
\sum_{j_{\Gamma_{L}}} \mathcal{E}_{j}^{ \pm n} \oint_{\gamma_{L}}\left(\hat{\mathbf{n}}_{L}(\mathbf{R}) \times \bar{\tau}_{i}(\mathbf{R})\right) \cdot \bar{\tau}_{j}\left(\mathbf{R}^{\prime}\right) d \Gamma_{L}^{\prime} \\
=\sum_{j=1}^{M_{L}} 2 \sqrt{Z_{n}} \oint_{\Gamma_{L}} e^{\mp \gamma_{n} z^{\prime}}\left[\mathbf{e}_{t}^{n}\left(\mathbf{r}^{\prime}\right) \pm \mathbf{e}_{z}^{n}\left(\mathbf{r}^{\prime}\right)\right] \cdot\left(\hat{\mathbf{n}}_{L}\left(\mathbf{R}^{\prime}\right) \times \bar{\tau}_{i}\left(\mathbf{R}^{\prime}\right)\right) d \Gamma_{L}^{\prime} \\
-2 j k_{0} Z_{0} \sum_{j_{\Gamma_{L}}} \mathcal{H}_{j}^{ \pm n} \oint_{\Gamma_{L}} \oint_{\Gamma_{L}}\left(\hat{\mathbf{n}}_{L}(\mathbf{R}) \times \bar{\tau}_{j}(\mathbf{R})\right) \cdot \mathbb{G}_{E}\left(\mathbf{R}, \mathbf{R}^{\prime}\right) \cdot\left(\hat{\mathbf{n}}_{L}\left(\mathbf{R}^{\prime}\right) \times \bar{\tau}_{i}\left(\mathbf{R}^{\prime}\right)\right) d \Gamma_{L} d \Gamma_{L}^{\prime} \\
+2 \sum_{j_{\Gamma_{L}}} \mathcal{E}_{j}^{ \pm n} \oint_{\Gamma_{L}} \oint_{\Gamma_{L}}\left(\hat{\mathbf{n}}_{L}(\mathbf{R}) \times \bar{\tau}_{j}(\mathbf{R})\right) \cdot \mathbb{G}_{H}\left(\mathbf{R}, \mathbf{R}^{\prime}\right) \cdot\left(\hat{\mathbf{n}}_{L}\left(\mathbf{R}^{\prime}\right) \times \bar{\tau}_{i}\left(\mathbf{R}^{\prime}\right)\right) d \Gamma_{L} d \Gamma_{L}^{\prime}
\end{array}
$$

\subsection{Solving the Volume Magnetic Fields and Surface Tangential Electric Fields}

Two linked matrix equations (2.17) and (2.20) are derived in the previous section. In matrix form these are

$$
\left[B_{E}\right]\left\{\mathcal{E}_{\Gamma_{L}}^{ \pm n}\right\}=\left\{\mathbf{e}^{ \pm n}\right\}+\left[M_{E}\right]\left\{\mathcal{H}_{\Gamma_{L}}^{ \pm n}\right\}+\left[M_{H}\right]\left\{\mathcal{E}_{\Gamma_{L}}^{ \pm n}\right\}
$$

and

$$
\left([S]-k_{0}^{2}[T]\right)\left\{\mathcal{H}^{ \pm n}\right\}=j k_{0} Y_{0}\left[B_{E}\right]\left\{\mathcal{E}_{\Gamma_{L}}^{ \pm n}\right\}
$$

These simultaneous equations can be solved for $\left\{\mathcal{H}^{ \pm n}\right\}$, the magnetic field coefficients of $\Omega_{L}$ including the surface magnetic field degrees of freedom $\left\{\mathcal{H}_{\Gamma_{L}}^{ \pm n}\right\}$, and the surface electric field degrees of freedom $\left\{\mathcal{E}_{\Gamma_{L}}^{ \pm n}\right\}$.

The elemental matrices to be constructed numerically are ${ }^{1}$

$$
\left[B_{E}\right]_{i l}^{(e l)}=\int_{\Gamma_{L}}\left(\hat{\mathbf{n}}_{L} \times \bar{\tau}_{i}\right) \cdot \bar{\tau}_{l} d \Gamma_{L}=\hat{\mathbf{n}}_{L} \cdot \int_{\Gamma_{L}} \bar{\tau}_{i} \times \bar{\tau}_{l} d \Gamma_{L}
$$

${ }^{1}$ Noting that the FE indices $i$ and $j$ were replaced by $i$ and $l$, to avoid notational difficulties in chapter 3 . 


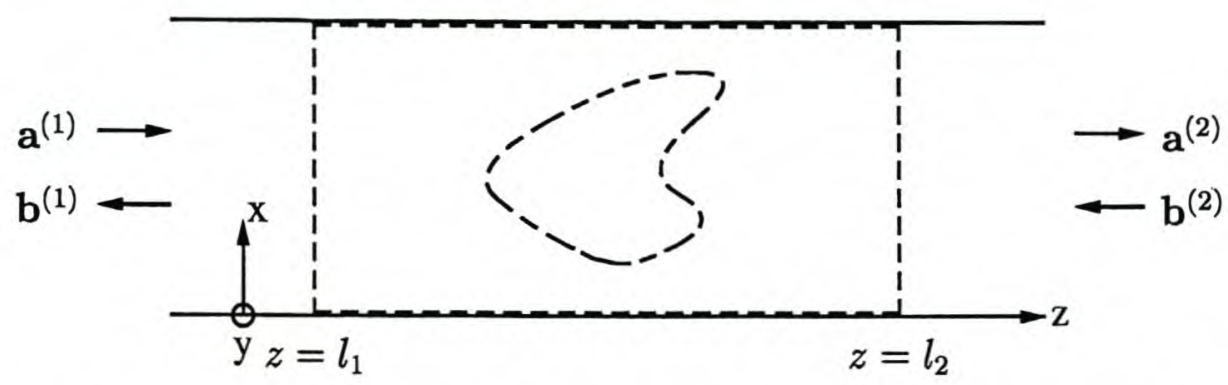

Figure 2.2: A schematic diagram representing the scattering coefficients $\mathbf{a}^{(1)}, \mathbf{a}^{(2)}$, $\mathbf{b}^{(1)}$ and $\mathbf{b}^{(2)}$. Note the deviation from the usual definition of $\mathbf{a}$ and $\mathbf{b}$.

$$
\begin{gathered}
{[S]_{i l}^{(e l)}=\int_{\Omega_{L}} \frac{1}{\epsilon_{r}}\left(\nabla \times \bar{\tau}_{i}\right) \cdot\left(\nabla \times \bar{\tau}_{l}\right) d \Omega} \\
{[T]_{i l}^{(e l)}=\int_{\Omega_{L}} \mu_{r} \bar{\tau}_{i} \cdot \bar{\tau}_{l} d \Omega} \\
\left\{\mathbf{e}^{ \pm n}\right\}_{i}^{(e l)}=2 \sqrt{Z_{n}} \int_{\Gamma_{L}}\left[\mathbf{e}_{t}^{n}\left(\mathbf{r}^{\prime}\right) \pm \mathbf{e}_{z}^{n}\left(\mathbf{r}^{\prime}\right)\right] \cdot\left[\hat{\mathbf{n}}_{L}\left(\mathbf{R}^{\prime}\right) \times \bar{\tau}_{i}\left(\mathbf{R}^{\prime}\right)\right] e^{\mp \gamma_{n} z^{\prime}} d \Gamma_{L}^{\prime} \\
{\left[M_{E}\right]_{i j}^{(e l)}=-2 j k_{0} Z_{0} \int_{\Gamma_{L}} \int_{\Gamma_{L}}\left[\hat{\mathbf{n}}_{L}(\mathbf{R}) \times \bar{\tau}_{j}(\mathbf{R})\right] \cdot \mathbb{G}_{E}\left(\mathbf{R}, \mathbf{R}^{\prime}\right) \cdot\left[\hat{\mathbf{n}}_{L}\left(\mathbf{R}^{\prime}\right) \times \bar{\tau}_{i}\left(\mathbf{R}^{\prime}\right)\right] d \Gamma_{L} d \Gamma_{L}^{\prime}} \\
\left.\left[M_{H}\right]_{i j}^{(e l)}=2 \int_{\Gamma_{L}} \int_{\Gamma_{L}}\left[\hat{\mathbf{n}}_{L}(\mathbf{R}) \times \bar{\tau}_{j}(\mathbf{R})\right] \cdot \mathbb{G}_{H}\left(\mathbf{R}, \mathbf{R}^{\prime}\right) \cdot\left[\hat{\mathbf{n}}_{L}\left(\mathbf{R}^{\prime}\right)\right) \times \bar{\tau}_{i}\left(\mathbf{R}^{\prime}\right)\right] d \Gamma_{L} d \Gamma_{L}^{\prime}
\end{gathered}
$$

The $[S]$ and $[T]$ matrices are FE matrices, and are typical to FE problems. $\left[B_{E}\right]$ is also a FE matrix, but forms the connection between the inner and outer part of the scatterer and waveguide. The remaining matrices, $\left[M_{E}\right]$ and $\left[M_{H}\right]$, are similar to method of moment matrices and are dense. $\left\{e^{ \pm n}\right\}$ is the excitation vector.

A matrix solution yields the values of the coefficients and enable the determination of the electric field inside the waveguide as well as the scattering parameters.

\subsection{The Generalized Scattering Matrix}

The scattering parameters are to be determined for an object or multiply disconnected objects placed in a section of waveguide. The length of the section of waveguide is determined by two reference planes $z=l_{1}$ and $z=l_{2}$ defined in figure 2.2. The possible $M$ incident modes from the left (magnitude and phase) are represented by the vector $\mathbf{a}^{(1)}=\left[a_{1}^{(1)}, a_{2}^{(1)}, \ldots, a_{M}^{(1)}\right]$. Similarly $\mathbf{b}^{(2)}=\left[b_{1}^{(2)}, b_{2}^{(2)}, \ldots, b_{M}^{(2)}\right]$ are the $M$ possible incident modes from the right. The scattered modes are represented by $b^{(1)}$ traveling in 
the negative $\mathbf{z}$-direction and $\mathbf{a}^{(2)}$ traveling in the positive $\mathbf{z}$-direction. These are defined at the origin $z=0$ and must be referred to the reference planes $z=l_{1}$ and $z=l_{2}$ respectively, through a phase shift dependent on the distance of these planes from the origin. S-parameters obtained should be accompanied by the values of $l_{1}$ and $l_{2}$ as these determine the phase of the S-parameters.

The two port scattering parameters are ratios of the $\mathbf{a}$ and $\mathbf{b}$ vectors

$$
\left[\begin{array}{l}
\mathbf{b}^{(1)} \\
\mathbf{a}^{(2)}
\end{array}\right]=\left[\begin{array}{ll}
\mathbf{S}_{11} & \mathbf{S}_{12} \\
\mathbf{S}_{21} & \mathbf{S}_{22}
\end{array}\right]\left[\begin{array}{l}
\mathbf{a}^{(1)} \\
\mathbf{b}^{(2)}
\end{array}\right]
$$

noting the deviation from the usual convention for the vectors $\mathbf{a}$ and $\mathbf{b}$ for port 2 .

To determine the S-parameters, single n-th mode excitation from one side of the obstacle is considered, i.e. $\mathbf{a}^{(1)}=\left[0, \ldots, a_{n}^{(1)+}, \ldots, 0\right]$ (incident from the left) or $\mathbf{b}^{(2)}=$ $\left[0, \ldots, b_{n}^{(2)-}, \ldots, 0\right]$ (incident from the right). The entire GSM matrix can be constructed by varying $n$.

\subsubsection{The Generalized Scattering Parameter $S_{21}^{(m n)}$}

Excitation is a unit normalized $n$-th mode from the left. The E-field at $\Gamma_{2}$ (see figure 2.1) due to the $\mathrm{n}$-th mode excitation and scattering from the waveguide obstacle according to $(2.10)$ is

$$
\begin{aligned}
\left.\mathbf{E}^{+n}\left(\mathbf{R}^{\prime}\right)\right|_{z^{\prime}=l_{2}} & =\sqrt{Z_{n}}\left[\mathbf{e}_{t}^{n}\left(\mathbf{r}^{\prime}\right)+\mathbf{e}_{z}^{n}\left(\mathbf{r}^{\prime}\right)\right] e^{-\gamma_{n} l_{2}} \\
& +\oint_{\Gamma_{L}}-\left.j k_{0} Z_{0} \sum_{j_{\Gamma_{L}}} \mathcal{H}_{j}^{+n}\left[\hat{\mathbf{n}}_{L} \times \bar{\tau}_{j}(\mathbf{R})\right] \cdot \mathbb{G}_{E}\left(\mathbf{R}, \mathbf{R}^{\prime}\right)\right|_{z^{\prime}=l_{2}} d \Gamma_{L} \\
& +\left.\oint_{\Gamma_{L}} \sum_{j_{\Gamma_{L}}} \mathcal{E}_{j}^{+n}\left[\hat{\mathbf{n}}_{L} \times \bar{\tau}_{j}(\mathbf{R})\right] \cdot \mathbb{G}_{H}\left(\mathbf{R}, \mathbf{R}^{\prime}\right)\right|_{z^{\prime}=l_{2}} d \Gamma_{L}
\end{aligned}
$$

Alternatively from (2.1), the same E-field at $\Gamma_{2}$ consists of an infinite sum of waveguide modes (some propagating, others evanescent) traveling in the positive $\mathrm{z}$ direction

$$
\left.\mathbf{E}^{(2)}\left(\mathbf{R}^{\prime}\right)\right|_{z^{\prime}=l_{2}}=\sum_{k=1}^{\infty} \sqrt{Z_{k}} a_{k}^{(2)}\left[\mathbf{e}_{t}^{k}\left(\mathbf{r}^{\prime}\right)+\mathbf{e}_{z}^{k}\left(\mathbf{r}^{\prime}\right)\right] e^{-\gamma_{k} l_{2}}
$$

The representations (2.30) and (2.31) are equivalent at any point $\mathbf{R}^{\prime}$ on $\Gamma_{2}$.

Taking the dot-product of (2.30) and (2.31) with an $m$-th mode transverse field $\mathbf{e}_{t}^{m}\left(\mathbf{r}^{\prime}\right)$; equating the expressions and integrating over the waveguide cross-section $\Gamma_{2}$ and using 
(2.5), one finds

$$
\begin{aligned}
a_{m}^{(2)} & =\delta_{m n} \frac{\sqrt{Z_{n}}}{\sqrt{Z_{m}}} e^{-\gamma_{n} l_{2}+\gamma_{m} l_{2}} \\
& +\sqrt{Y_{m}} e^{\gamma_{m} l_{2}} \int_{\Gamma_{2}} \mathbf{e}_{t}^{m}\left(\mathbf{r}^{\prime}\right) \cdot \oint_{\Gamma_{L}}-\left.j k_{0} Z_{0} \sum_{j \Gamma_{L}} \mathcal{H}_{j}^{+n}\left[\hat{\mathbf{n}}_{L} \times \bar{\tau}_{j}(\mathbf{R})\right] \cdot \mathbb{G}_{E}\left(\mathbf{R}, \mathbf{R}^{\prime}\right)\right|_{z^{\prime}=l_{2}} d \Gamma_{L} d x^{\prime} d y^{\prime} \\
& +\left.\sqrt{Y_{m}} e^{\gamma_{m} l_{2}} \int_{\Gamma_{2}} \mathbf{e}_{t}^{m}\left(\mathbf{r}^{\prime}\right) \cdot \oint_{\Gamma_{L}} \sum_{j_{\Gamma_{L}}} \mathcal{E}_{j}^{+n}\left[\hat{\mathbf{n}}_{L} \times \bar{\tau}_{j}(\mathbf{R})\right] \cdot \mathbb{G}_{H}\left(\mathbf{R}, \mathbf{R}^{\prime}\right)\right|_{z^{\prime}=l_{2}} d \Gamma_{L} d x^{\prime} d y^{\prime}
\end{aligned}
$$

$S_{21}^{(m n)}$ is defined as the ratio of the transmitted m-th mode amplitude referred to $\Gamma_{2}$ : $\sqrt{z_{m}} a_{m}^{(2)} e^{-\gamma_{m} l_{2}}$ to the incident unit $\mathrm{n}$-th mode amplitude referred to $\Gamma_{1}: \sqrt{z_{n}} e^{-\gamma_{n} l_{1}}$, giving

$$
S_{21}^{(m n)}=\frac{\sqrt{Z_{m}}}{\sqrt{Z_{n}}} a_{m}^{(2)} e^{-\gamma_{m} l_{2}+\gamma_{n} l_{1}}
$$

$$
\begin{array}{r}
S_{21}^{m n}=e^{-\gamma_{n} l_{2}+\gamma_{n} l_{1}} \delta_{m n}+\sqrt{Y_{n}} e^{\gamma_{n} l_{1}} \int_{\Gamma_{2}} \mathbf{e}_{t}^{m}\left(\mathbf{r}^{\prime}\right) \\
\left.\oint_{\Gamma_{L}}\left(-j k_{0} Z_{0}\right) \sum_{j_{\Gamma_{L}}}\left[\mathcal{H}_{j}^{+n} \hat{\mathbf{n}}_{L} \times \bar{\tau}_{j}(\mathbf{R})\right] \cdot \mathbb{G}_{E}\left(\mathbf{R}, \mathbf{R}^{\prime}\right)\right|_{z^{\prime}=l_{2}} d \Gamma_{L} d x^{\prime} d y^{\prime} \\
+\sqrt{Y_{n}} e^{\gamma_{n} l_{1}} \int_{\Gamma_{2}} \mathbf{e}_{t}^{m}\left(\mathbf{r}^{\prime}\right) \\
\left.\cdot \oint_{\Gamma_{L}} \sum_{j_{\Gamma_{L}}}\left[\mathcal{E}_{j}^{+n} \hat{\mathbf{n}}_{L} \times \bar{\tau}_{j}(\mathbf{R})\right] \cdot \mathbb{G}_{H}\left(\mathbf{R}, \mathbf{R}^{\prime}\right)\right|_{z^{\prime}=l_{2}} d \Gamma_{L} d x^{\prime} d y^{\prime}
\end{array}
$$

$S_{21}^{(m n)}=e^{-\gamma_{n}\left(l_{2}-l_{1}\right)}$ when $m=n$ and there is no obstacle in the waveguide.

\subsubsection{The Generalized Scattering Parameter $S_{11}^{(m n)}$}

Excitation is a unit normalized n-th mode from the left. The E-field is expressed according to (2.10) in terms of the $n$-th mode excitation at $\Gamma_{1}$ (see figure 2.1) as

$$
\begin{aligned}
\left.\mathbf{E}^{+n}\left(\mathbf{R}^{\prime}\right)\right|_{z^{\prime}=l_{1}} & =\sqrt{Z_{n}}\left[\mathbf{e}_{t}^{n}\left(\mathbf{r}^{\prime}\right)+\mathbf{e}_{z}^{n}\left(\mathbf{r}^{\prime}\right)\right] e^{-\gamma_{n} l_{1}} \\
& +\oint_{\Gamma_{L}}-\left.j k_{0} Z_{0} \sum_{j_{\Gamma_{L}}} \mathcal{H}_{j}^{+n}\left[\hat{\mathbf{n}}_{L} \times \bar{\tau}_{j}(\mathbf{R})\right] \cdot \mathbb{G}_{E}\left(\mathbf{R}, \mathbf{R}^{\prime}\right)\right|_{z^{\prime}=l_{1}} d \Gamma_{L} \\
& +\left.\oint_{\Gamma_{L}} \sum_{j_{\Gamma_{L}}} \mathcal{E}_{j}^{+n}\left[\hat{\mathbf{n}}_{L} \times \bar{\tau}_{j}(\mathbf{R})\right] \cdot \mathbb{G}_{H}\left(\mathbf{R}, \mathbf{R}^{\prime}\right)\right|_{z^{\prime}=l_{1}} d \Gamma_{L}
\end{aligned}
$$


The E-field is expressed as a sum of the $\mathrm{n}$-th mode excitation, negative-traveling and evanescent modes at $\Gamma_{1}$ :

$$
\left.\mathbf{E}^{(1)}(\mathbf{R})\right|_{z^{\prime}=l_{1}}=\sqrt{Z_{n}} e^{-\gamma_{n} l_{1}}\left[\mathbf{e}_{t}^{n}\left(\mathbf{r}^{\prime}\right)+\mathbf{e}_{z}^{n}\left(\mathbf{r}^{\prime}\right)\right]+\sum_{k=1}^{\infty} \sqrt{Z_{k}}\left\{b_{k}^{(1)} e^{\gamma_{k} l_{1}}\left[\mathbf{e}_{t}^{k}\left(\mathbf{r}^{\prime}\right)-\mathbf{e}_{z}^{k}\left(\mathbf{r}^{\prime}\right)\right]\right\}
$$

Following a similar procedure as in section 2.8.1, but integrating over the waveguide cross-section $\Gamma_{1}$ instead of $\Gamma_{2}$, one finds

$$
\begin{aligned}
b_{m}^{(1)} & =\sqrt{Y_{m}} e^{-\gamma_{m} l_{1}} \int_{\Gamma_{1}} \mathbf{e}_{t}^{m}\left(\mathbf{r}^{\prime}\right) \cdot \oint_{\Gamma_{L}}-\left.j k_{0} Z_{0} \sum_{j_{L}} \mathcal{H}_{j}^{+n}\left[\hat{\mathbf{n}}_{L} \times \bar{\tau}_{j}(\mathbf{R})\right] \cdot \mathbb{G}_{E}\left(\mathbf{R}, \mathbf{R}^{\prime}\right)\right|_{z^{\prime}=l_{1}} d \Gamma_{L} d x^{\prime} d y^{\prime} \\
& +\left.\sqrt{Y_{m}} e^{-\gamma_{m} l_{1}} \int_{\Gamma_{1}} \mathbf{e}_{t}^{m}\left(\mathbf{r}^{\prime}\right) \cdot \oint_{\Gamma_{L}} \sum_{j_{\Gamma_{L}}} \mathcal{E}_{j}^{+n}\left[\hat{\mathbf{n}}_{L} \times \bar{\tau}_{j}(\mathbf{R})\right] \cdot \mathbb{G}_{H}\left(\mathbf{R}, \mathbf{R}^{\prime}\right)\right|_{z^{\prime}=l_{1}} d \Gamma_{L} d x^{\prime} d y^{\prime}
\end{aligned}
$$

$S_{11}^{(m n)}$ is defined as the ratio of the reflected $\mathrm{m}$-th mode amplitude and phase at $\Gamma_{1}$ : $\sqrt{z_{m}} b_{m}^{(1)} e^{\gamma_{m} l_{1}}$ and the incident n-th mode amplitude and phase at $\Gamma_{1}: \sqrt{z_{n}} e^{-\gamma_{n} l_{1}}$ leading to

$$
\begin{array}{r}
S_{m n}^{(11)}=\sqrt{Y_{n}} e^{\gamma_{n} l_{1}} \int_{\Gamma_{1}} \mathbf{e}_{t}^{m}\left(\mathbf{r}^{\prime}\right) \\
\left.\cdot \oint_{\Gamma_{L}}\left(-j k_{0} Z_{0}\right) \sum_{j_{\Gamma_{L}}} \mathcal{H}_{j}^{+n}\left[\hat{\mathbf{n}}_{L} \times \bar{\tau}_{j}(\mathbf{R})\right] \cdot \mathbb{G}_{E}\left(\mathbf{R}, \mathbf{R}^{\prime}\right)\right|_{z^{\prime}=l_{1}} d \Gamma_{L} d x^{\prime} d y^{\prime} \\
+\sqrt{Y_{n}} e^{\gamma_{n} l_{1}} \int_{\Gamma_{1}} \mathbf{e}_{t}^{m}\left(\mathbf{r}^{\prime}\right) \\
\left.\cdot \oint_{\Gamma_{L}} \sum_{j_{\Gamma_{L}}} \mathcal{E}_{j}^{+n}\left[\hat{\mathbf{n}}_{L} \times \bar{\tau}_{j}(\mathbf{R})\right] \cdot \mathbb{G}_{H}\left(\mathbf{R}, \mathbf{R}^{\prime}\right)\right|_{z^{\prime}=l_{1}} d \Gamma_{L} d x^{\prime} d y^{\prime}
\end{array}
$$

\subsubsection{The Generalized Scattering Parameter $S_{12}^{(m n)}$}

Following a similar procedure as for $S_{21}^{(m n)}$, but with normalized n-th mode excitation from the right, 


$$
\begin{array}{r}
S_{12}^{m n}=e^{\gamma_{n} l_{2}-\gamma_{n} l_{1}} \delta_{m n}+\sqrt{Y_{n}} e^{-\gamma_{n} l_{2}} \int_{\Gamma_{2}} \mathbf{e}_{t}^{m}\left(\mathbf{r}^{\prime}\right) \\
\left.\cdot \oint_{\Gamma_{L}}\left(-j k_{0} Z_{0}\right) \sum_{j_{\Gamma_{L}}}\left[\mathcal{H}_{j}^{-n} \hat{\mathbf{n}}_{L} \times \bar{\tau}_{j}(\mathbf{R})\right] \cdot \mathbb{G}_{E}\left(\mathbf{R}, \mathbf{R}^{\prime}\right)\right|_{z^{\prime}=l_{1}} d \Gamma_{L} d x^{\prime} d y^{\prime} \\
+\sqrt{Y_{n}} e^{-\gamma_{n} l_{2}} \int_{\Gamma_{2}} \mathbf{e}_{t}^{m}\left(\mathbf{r}^{\prime}\right) \\
\left.\cdot \oint_{\Gamma_{L}} \sum_{j_{\Gamma_{L}}}\left[\mathcal{E}_{j}^{-n} \hat{\mathbf{n}}_{L} \times \bar{\tau}_{j}(\mathbf{R})\right] \cdot \mathbb{G}_{H}\left(\mathbf{R}, \mathbf{R}^{\prime}\right)\right|_{z^{\prime}=l_{1}} d \Gamma_{L} d x^{\prime} d y^{\prime}
\end{array}
$$

\subsubsection{The Generalized Scattering Parameter $S_{22}^{(m n)}$}

Following a similar procedure as for $S_{11}^{(m n)}$, but with normalized n-th mode excitation from the right,

$$
\begin{array}{r}
S_{m n}^{(22)}=\sqrt{Y_{n}} e^{-\gamma_{n} l_{2}} \int_{\Gamma_{2}} \mathbf{e}_{t}^{m}\left(\mathbf{r}^{\prime}\right) \\
\left.\cdot \oint_{\Gamma_{L}}\left(-j k_{0} Z_{0}\right) \sum_{j_{\Gamma_{L}}} \mathcal{H}_{j}^{-n}\left[\hat{\mathbf{n}}_{L} \times \bar{\tau}_{j}(\mathbf{R})\right] \cdot \mathbb{G}_{E}\left(\mathbf{R}, \mathbf{R}^{\prime}\right)\right|_{z^{\prime}=l_{2}} d \Gamma_{L} d x^{\prime} d y^{\prime} \\
+\sqrt{Y_{n}} e^{-\gamma_{n} l_{2}} \int_{\Gamma_{2}} \mathbf{e}_{t}^{m}\left(\mathbf{r}^{\prime}\right) \\
\left.\cdot \oint_{\Gamma_{L}} \sum_{j_{\Gamma_{L}}} \mathcal{E}_{j}^{-n}\left[\hat{\mathbf{n}}_{L} \times \bar{\tau}_{j}(\mathbf{R})\right] \cdot \mathbb{G}_{H}\left(\mathbf{R}, \mathbf{R}^{\prime}\right)\right|_{z^{\prime}=l_{2}} d \Gamma_{L} d x^{\prime} d y^{\prime}
\end{array}
$$

\subsection{Conclusion}

In this chapter, a mathematical representation of the Huygens' principle in waveguide is described as the basis for a new technique to solve scattering problems in waveguide by discretizing the volume of the scatterer only. The internal fields of the scatterer are linked to the empty waveguide exterior of the scatterer by the surface fields of the scattering body. The surface integrations required are similar to volume MoM formulations, but the integrations are confined to a surface only and not the entire volume of the scattering body, even when dielectric bodies are considered. The waveguide dyadic functions are infinite sums of waveguide modal functions that represent the section of empty waveguide in the absence of any objects inside the waveguide. These functions contain discontinuities that require special care in the integration of these functions. The insertion of the factor $T$ and the derivations of S-parameter expressions are not found in [33]. This concludes the summary of the method suggested in [33]. 


\section{Chapter 3}

\section{Implementation of Posts in Waveguide}

\subsection{Introduction}

This chapter introduces the implementational challenges presented by the technique for the problem of scattering by objects in a uniform hollow waveguide. Important aspects are the discretization of the scatterer, the finite element basis set and matrices, the evaluation of the double surface integrals over the surface patches and the solution of the coupled matrix equations.

The discretization of the scatterer, which is dictated by the typical geometry of the scattering object, must be considered first, as this will influence the choice of a basis set. The volume elements determine the shape of the surface elements on the outer surface area of the scatterer, where either triangular or rectangular elements are typically used. The order of approximation of the fields associated with the basis set must be considered, while results may be improved with a refinement of the mesh or by employing a higher order basis set. For this implementation a basis set was adapted from the literature to suit the specific requirements of the problem. Once this choice is made, the elemental finite element (FE) matrices can be derived.

The link between the inner volume and the empty section of waveguide is made via the surface electric fields. A connection matrix, $\left[B_{E}\right]$, introduced in section 2.7 of chapter 2, is responsible for linking the inner volume magnetic fields, represented by a FE discretization, and the surface tangential electric fields. A strategy to confirm the validity of the $\mathrm{FE}$ implementation and connection matrix approach was devised for separate testing of the FE implementation and the connection matrix, which confirms the validity of the implementations. The surface patches support magnetic and electric fields, of which the tangential components act as sources for the scattered electric field inside the hollow sections of waveguide. 


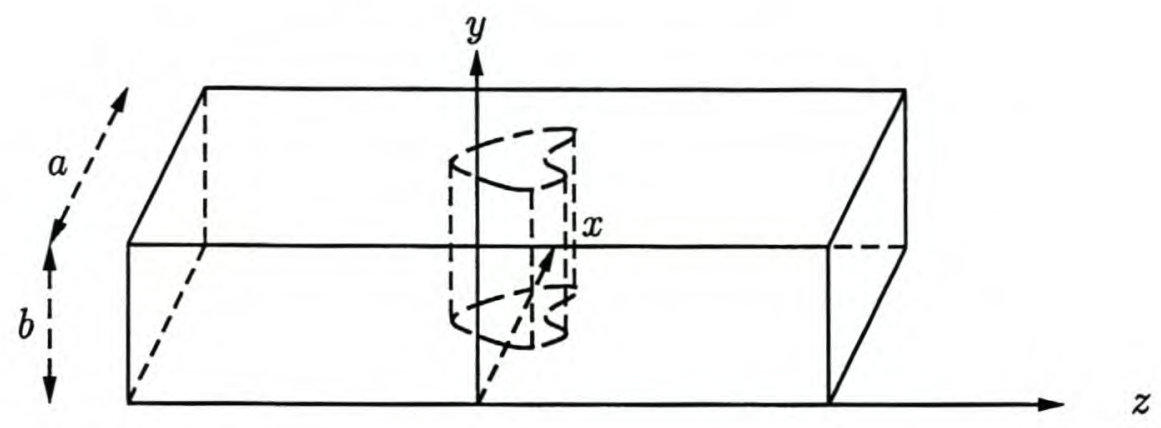

Figure 3.1: A homogeneous cylindrical post, either dielectric, magnetic or conducting, extending between the broad faces of a rectangular $T E_{10}$ waveguide. The broad wall of the waveguide has dimension a, while the narrow wall has the dimension $b$.

In the process of calculating the scattered fields, double surface integrals involving dyadic waveguide Green functions present convergence difficulties that may be circumvented by a numerical integration scheme with non-coincident integration points. Finally, the solution of the matrix equation requires manipulation of the matrices to obtain an accurate solution.

This chapter contains the main contributions of the thesis, excluding contributions from other sources as referenced. These are the adapted finite element basis sets, the derivation of the elemental matrices and the numerical integration scheme.

\subsection{Simplifications}

For the purpose of demonstration, it was decided to model a homogeneous conducting or dielectric post in a standard $T E_{10}$ waveguide, (figure 3.1). All $T M_{m n}$ modes may be excluded from our analysis as well as $T E_{m n}$ modes with $n \neq 0$, reducing the computational burden by ruling out any y-variation. This simplifies to a single infinite summation in the waveguide Green functions: now only sums of $T E_{n 0}$ modes. Since all $T M_{n m}$ modes include a y-variation, these are not excited with the geometries considered here. Such a restriction also allows the employment of prismatic finite elements (figure 3.2). A further simplification that can be made is for centered posts; in these cases only even modes, i.e. $T E_{10}, T E_{30}$ etc., are excited. 


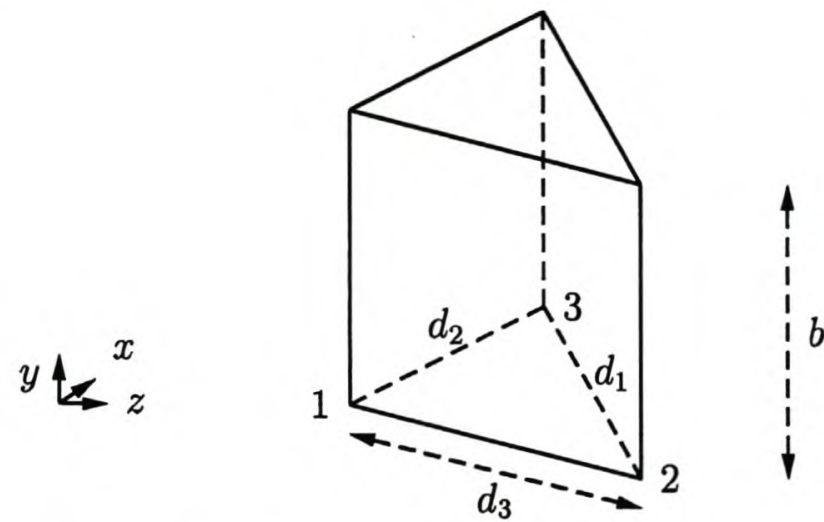

Figure 3.2: The prismatic finite element geometry. Side face 1 is defined by nodes 2 and 3. Side face 2 is defined by nodes 3 and 1 , and side face 3 is defined by nodes 1 and 2 . The lengths, $d_{i}$, with $i=1,2,3$, of each side face are indicated. The element extends from $y=0$ to $y=b$, with $b$ the height of the waveguide, as indicated in

figure 3.1.

\subsection{Finite Element Discretization}

\subsubsection{Prismatic elements}

A basis function set for 3D prismatic elements is found in [39, 22]. Transformed to our coordinate system the basis functions are given by

$$
\begin{aligned}
& \mathbf{W}_{1}=d_{1}\left(\lambda_{2} \nabla \lambda_{3}-\lambda_{3} \nabla \lambda_{2}\right) \frac{y}{b} \\
& \mathbf{W}_{2}=d_{2}\left(\lambda_{3} \nabla \lambda_{1}-\lambda_{1} \nabla \lambda_{3}\right) \frac{y}{b} \\
& \mathbf{W}_{3}=d_{3}\left(\lambda_{1} \nabla \lambda_{2}-\lambda_{2} \nabla \lambda_{1}\right) \frac{y}{b} \\
& \mathbf{W}_{4}=d_{1}\left(\lambda_{2} \nabla \lambda_{3}-\lambda_{3} \nabla \lambda_{2}\right)\left(1-\frac{y}{b}\right) \\
& \mathbf{W}_{5}=d_{2}\left(\lambda_{3} \nabla \lambda_{1}-\lambda_{1} \nabla \lambda_{3}\right)\left(1-\frac{y}{b}\right) \\
& \mathbf{W}_{6}=d_{3}\left(\lambda_{1} \nabla \lambda_{2}-\lambda_{2} \nabla \lambda_{1}\right)\left(1-\frac{y}{b}\right) \\
& \mathbf{K}_{1}=\widehat{y} \lambda_{1} \\
& \mathbf{K}_{2}=\widehat{y} \lambda_{2} \\
& \mathbf{K}_{3}=\widehat{y} \lambda_{3}
\end{aligned}
$$

where $\lambda_{i}, i=1,2,3$ are the 2D simplex coordinates for triangular elements [21]. The extension to $3 \mathrm{D}$ is made by using $\mathrm{y}$ as the fourth local coordinate on an element. These prismatic elements allow a $2 \mathrm{D}$ discretization of the obstacle to be extended to 


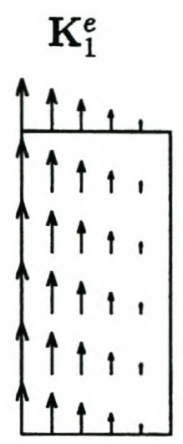

$\mathbf{K}_{2}^{e}$
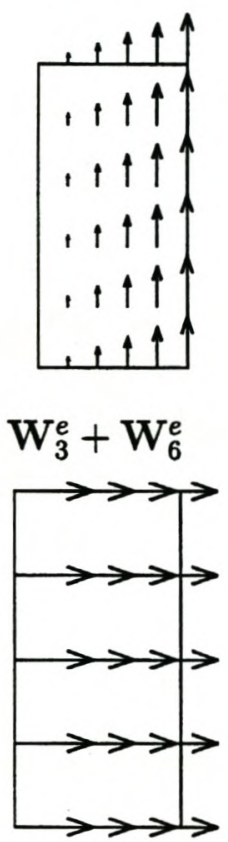

$\hat{\mathbf{n}} \times \mathbf{K}_{1}^{e}$

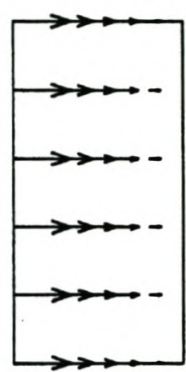

$\hat{\mathbf{n}} \times \mathbf{K}_{2}^{e}$

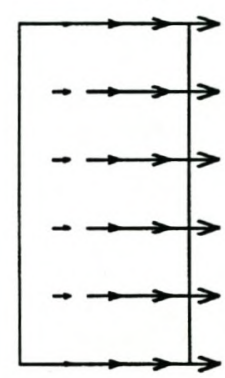

$\hat{\mathbf{n}} \times\left(\mathbf{W}_{3}^{e}+\mathbf{W}_{6}^{e}\right)$

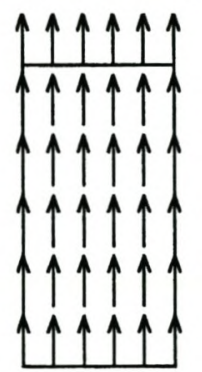

Figure 3.3: Prismatic basis function on a prismatic element face, basis set (3.2) in column 1. Column 2 shows the cross-product of the normal vector $\hat{\mathbf{n}}$ with each basis function that is required for surface integration over the surface patch area. 


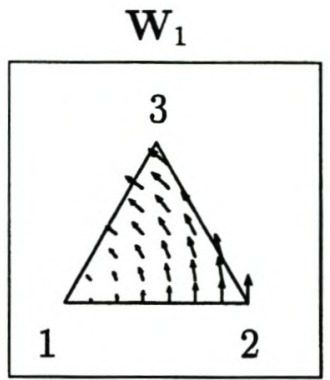

$\mathbf{M}_{1}$
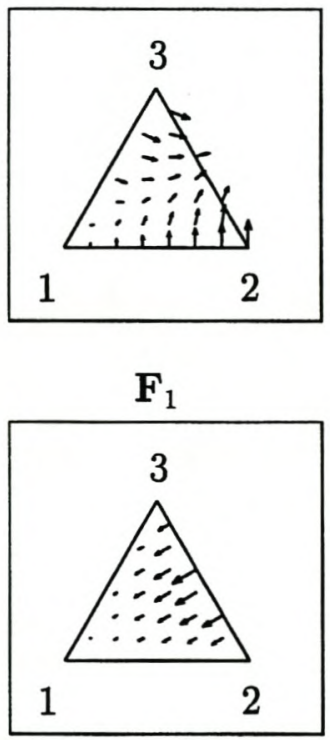
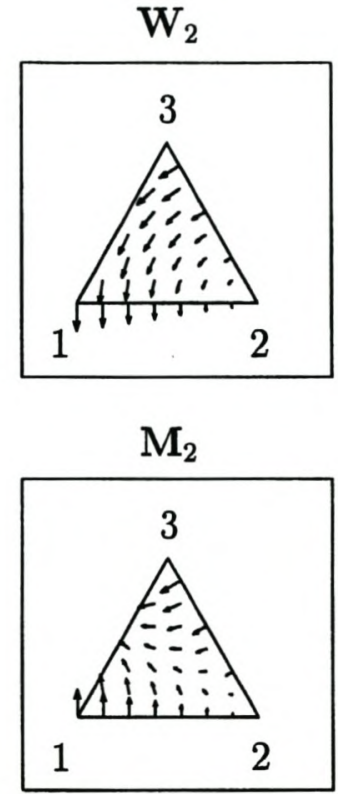

$\mathbf{F}_{2}$

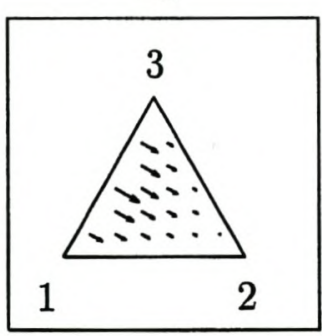

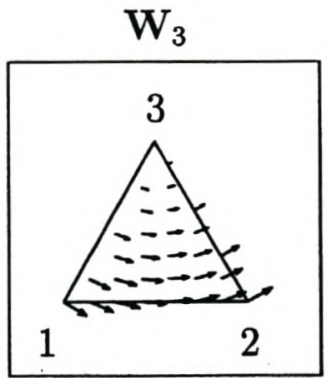

$\mathbf{M}_{3}$

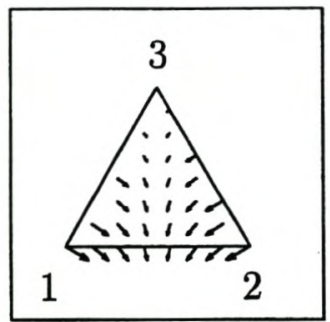

Figure 3.4: Transverse basis functions on triangular cross-section of prismatic element at $y=b$. The first three are for basis set (3.2), while the full set is required for basis set (3.3). 
3D by adding another dimension to the triangular discretization. It is most suitable for problems where the object cross-section is fairly uniform along the length of the object. For our purposes the linear variation along the y-direction of the element is not directly suitable for modelling scattering problems with no y-variation in the geometry or excitation. If implemented as is, linear dependence of matrix equations results. A solution to this problem is to reduce the linear basis function to a constant over the element. This may be seen as combining basis type $\mathbf{W}_{i}$ and type $\mathbf{W}_{i+3}$ with equal amplitude, the linear variation in $y$ removed, with

$$
\begin{aligned}
& \mathbf{W}_{1}=d_{1}\left(\lambda_{2} \nabla \lambda_{3}-\lambda_{3} \nabla \lambda_{2}\right) \\
& \mathbf{W}_{2}=d_{2}\left(\lambda_{3} \nabla \lambda_{1}-\lambda_{1} \nabla \lambda_{3}\right) \\
& \mathbf{W}_{3}=d_{3}\left(\lambda_{1} \nabla \lambda_{2}-\lambda_{2} \nabla \lambda_{1}\right) \\
& \mathbf{K}_{1}=\widehat{y} \lambda_{1} \\
& \mathbf{K}_{2}=\widehat{y} \lambda_{2} \\
& \mathbf{K}_{3}=\widehat{y} \lambda_{3}
\end{aligned}
$$

Each prismatic element now has a reduced set of 6 degrees of freedom associated with it. The basis functions are now suitable for problems that have both uniform geometry and excitation in the $\mathrm{y}$-variable. The transverse plane is the $\mathrm{x}-\mathrm{z}$ plane in figure 3.2, the approximation is the well-known CT/LN ${ }^{1}$ elements $[40,41]$, combined with linear basis functions in the vertical direction. In fact, CT/LN is a suitable description for the entire element basis set in (3.2).

The bases $\mathbf{W}_{i}$ and $\mathbf{M}_{\boldsymbol{i}}$ will model only magnetic fields (surface and volume), while $\mathrm{K}_{i}$ will model only surface electric fields, due to the excitation and the simplified geometry. As a result, it may be shown that from the basis set 3.2 the tangential magnetic field is modeled as constant over an element, while the tangential electric field is modeled as a linear function over a patch element. To increase the order of the magnetic field approximation, one needs to upgrade the transverse order of the basis functions from $\mathrm{CT} / \mathrm{LN}$ to $\mathrm{LT} / \mathrm{QN}^{2}$ functions.

For a LT/QN transverse approximation, the hierarchal basis functions for a 2D trian-

\footnotetext{
${ }^{1} \mathrm{CT} / \mathrm{LN}$ : constant tangential, linear normal, which refers to the order of approximation of the field by the set of basis functions, with respect to an element edge

${ }^{2} \mathrm{LT} / \mathrm{QN}$ : linear tangential, quadratic normal approximation with respect to an element edge
} 
gular element, found in $[41,42]$, are used to increase the order of the basis functions:

$$
\begin{aligned}
& \mathbf{W}_{1}=d_{1}\left(\lambda_{2} \nabla \lambda_{3}-\lambda_{3} \nabla \lambda_{2}\right) \\
& \mathbf{W}_{2}=d_{2}\left(\lambda_{3} \nabla \lambda_{1}-\lambda_{1} \nabla \lambda_{3}\right) \\
& \mathbf{W}_{3}=d_{3}\left(\lambda_{1} \nabla \lambda_{2}-\lambda_{2} \nabla \lambda_{1}\right) \\
& \mathbf{M}_{1}=d_{1}\left(\lambda_{2} \nabla \lambda_{3}+\lambda_{3} \nabla \lambda_{2}\right) \\
& \mathbf{M}_{2}=d_{2}\left(\lambda_{3} \nabla \lambda_{1}+\lambda_{1} \nabla \lambda_{3}\right) \\
& \mathbf{M}_{3}=d_{3}\left(\lambda_{1} \nabla \lambda_{2}+\lambda_{2} \nabla \lambda_{1}\right) \\
& \mathbf{F}_{1}=\lambda_{2} \lambda_{3} \nabla \lambda_{1} \\
& \mathbf{F}_{2}=\lambda_{1} \lambda_{3} \nabla \lambda_{2} \\
& \mathbf{K}_{1}=\widehat{y} \lambda_{1} \\
& \mathbf{K}_{2}=\widehat{y} \lambda_{2} \\
& \mathbf{K}_{3}=\widehat{y} \lambda_{3}
\end{aligned}
$$

From now onwards the entire 3D basis set in (3.3) will be referred to as LT/QN.

\subsubsection{The Finite Element Matrices}

Finite element matrices are assembled on a per element basis [21]. The elemental matrices for each element are assembled into the global FE matrices by considering the inter-element connectivity.

The following terms occur in the matrix entries and are defined by

$$
\begin{aligned}
& \mathbf{h}_{i l}=\nabla \lambda_{i} \times \nabla \lambda_{l} \\
& \mathbf{m}_{l}=2 A \nabla \times \hat{y} \lambda_{l} \\
& p_{i l}=\nabla \lambda_{i} \cdot \nabla \lambda_{l}
\end{aligned}
$$

where $A$ is the triangular cross-sectional area of an element. The identity $\int_{A} \lambda_{i} \lambda_{j} \lambda_{k} d A=$ $A \frac{i ! j ! k ! 2 !}{(i+j+k+2) !}$ is required for many of the entries below.

\section{Elemental S Matrix Entries}

For (3.2), the symmetrical $S$-matrix consists of four submatrices, the possible interactions between the two types of basis functions

$$
[S]_{C T / L N}^{(e l)}=\left[\begin{array}{ll}
S^{(W W)} & S^{(W K)} \\
& S^{(K K)}
\end{array}\right]
$$


where, in the interest of clarity, the entries for symmetric partners to off-diagonal matrix terms are left blank. These form a subset of the matrices for the higher order LT/QN basis set matrices, as the basis functions are hierarchal. For (3.3), the $S$-matrix consists of sixteen submatrices, the possible interactions between the four types of basis functions:

$$
[S]_{L T / Q N}^{(e l)}=\left[\begin{array}{cccc}
S^{(W W)} & S^{(W M)} & S^{(W F)} & S^{(W K)} \\
& S^{(M M)} & S^{(M F)} & S^{(M K)} \\
& & S^{(F F)} & S^{(F K)} \\
& & & S^{(K K)}
\end{array}\right]
$$

The individual entries are

$$
\begin{aligned}
S_{(i l)}^{(W W)} & =\int_{V}\left(\nabla \times \mathbf{W}_{i}\right) \cdot\left(\nabla \times \mathbf{W}_{l}\right) d V \\
& =4 d_{i} d_{l}\left[\nabla \lambda_{j} \times \nabla \lambda_{k}\right] \cdot\left[\nabla \lambda_{m} \times \nabla \lambda_{n}\right] \int_{V} d v \\
& =4 b A d_{i} d_{l} \mathbf{h}_{\mathbf{j k}} \cdot \mathbf{h}_{\mathbf{m n}} \\
S_{(i l)}^{(W M)} & =\int_{V}\left(\nabla \times \mathbf{W}_{i}\right) \cdot\left(\nabla \times \mathbf{M}_{l}\right) d V \\
& =0 \\
S_{(i l)}^{(W F)} & =\int_{V}\left(\nabla \times \mathbf{W}_{i}\right) \cdot\left(\nabla \times \mathbf{F}_{l}\right) d V(l \leq 2) \\
& =2 d_{i} \int_{V} \mathbf{h}_{j k} \cdot\left(\lambda_{m} \mathbf{h}_{j k} \cdot \mathbf{h}_{n l}+\lambda_{n} \mathbf{h}_{j k} \cdot \mathbf{h}_{m l}\right) d V \\
& =2 d_{i} b \frac{A}{3}\left(\mathbf{h}_{j k} \cdot \mathbf{h}_{n l}+\mathbf{h}_{j k} \cdot \mathbf{h}_{m l}\right) \\
S_{(i l)}^{(W K)} & =\int_{V}\left(\nabla \times \mathbf{W}_{i}\right) \cdot\left(\nabla \times \mathbf{K}_{l}\right) d V \\
& =2 d_{i}\left[\nabla \lambda_{j} \times \nabla \lambda_{k}\right] \cdot \frac{\mathbf{m}_{l}}{2 A} \int_{V} d v \\
& =b d_{i} \mathbf{h}_{\mathbf{j k}} \cdot \mathbf{m}_{1} \\
& =0 \\
S_{(i l)}^{(M M)}= & 0, S_{(i l)}^{(M F)}=0, S_{(i j)}^{(M K)}=0
\end{aligned}
$$




$$
\begin{aligned}
S_{(i l)}^{(K K)=} & \int_{V}\left(\nabla \times \mathbf{K}_{i}\right) \cdot\left(\nabla \times \mathbf{K}_{l}\right) d V \\
= & \frac{b}{4 A} \mathbf{m}_{i} \cdot \mathbf{m}_{l} \\
S_{(i l)}^{(F F)}= & \int_{V}\left(\nabla \times \mathbf{F}_{i}\right) \cdot\left(\nabla \times \mathbf{F}_{l}\right) d V \\
= & \int_{V}\left(\lambda_{k} \mathbf{h}_{j i}+\lambda_{j} \mathbf{h}_{k i}\right) \cdot\left(\lambda_{n} \mathbf{h}_{m l}+\lambda_{m} \mathbf{h}_{n l}\right) d V \\
= & \mathbf{h}_{j i} \cdot \mathbf{h}_{m l} \int_{V} \lambda_{k} \lambda_{n} d V+\mathbf{h}_{j i} \cdot \mathbf{h}_{n l} \int_{V} \lambda_{j} \lambda_{n} d V \\
& +\mathbf{h}_{k i} \cdot \mathbf{h}_{n l} \int_{V} \lambda_{j} \lambda_{m} d V \\
S_{(i l)}^{(K F)}= & \int_{V}\left(\nabla \times \mathbf{K}_{i}\right) \cdot\left(\nabla \times \mathbf{F}_{l}\right) d V \\
= & 0
\end{aligned}
$$

where $i, j, k=1,2,3$ are numbered in cyclic order:

\begin{tabular}{lll}
$i$ & $j$ & $k$ \\
\hline 1 & 2 & 3 \\
2 & 3 & 1 \\
3 & 1 & 2
\end{tabular}

similarly for $l, m$ and $n$.

\section{Elemental T Matrix Entries}

Similarly to the S-matrix and, once again, with the off-diagonal symmetrical entries left blank for clarity,

$$
[T]_{C T / L N}^{(e l)}=\left[\begin{array}{ll}
T^{(W W)} & T^{(W K)} \\
& T^{(K K)}
\end{array}\right]
$$

and

$$
[T]_{L T / Q N}^{(e l)}=\left[\begin{array}{cccc}
T^{(W W)} & T^{(W M)} & T^{(W F)} & T^{(W K)} \\
& T^{(M M)} & T^{(M F)} & T^{(M K)} \\
& & T^{(F F)} & T^{(F K)} \\
& & & T^{(K K)}
\end{array}\right]
$$


with

$$
\begin{aligned}
T_{(i l)}^{(W W)} & =\int_{V} \mathbf{W}_{i} \cdot \mathbf{W}_{l} d V \\
& =d_{i} d_{l} \int_{V}\left[\lambda_{j} \nabla \lambda_{k}-\lambda_{k} \nabla \lambda_{j}\right] \cdot\left[\lambda_{m} \nabla \lambda_{n}-\lambda_{n} \nabla \lambda_{m}\right] d V \\
& =d_{i} d_{l} b \int_{A} \lambda_{j} \lambda_{m} \mathbf{m}_{k} \cdot \mathbf{m}_{n}-\lambda_{j} \lambda_{n} \mathbf{m}_{k} \cdot \mathbf{m}_{m}-\lambda_{k} \lambda_{m} \mathbf{m}_{j} \cdot \mathbf{m}_{n}+\lambda_{n} \lambda_{k} \mathbf{m}_{j} \cdot \mathbf{m}_{m} d A \\
& =b d_{i} d_{l}\left\{g_{j m} \mathbf{m}_{k} \cdot \mathbf{m}_{n}-g_{j n} \mathbf{m}_{k} \cdot \mathbf{m}_{m}-g_{k m} \mathbf{m}_{j} \cdot \mathbf{m}_{n}+g_{k n} \mathbf{m}_{j} \cdot \mathbf{m}_{m}\right\}
\end{aligned}
$$

similarly

$$
\begin{aligned}
T_{(i l)}^{(W M)} & =\int_{V} \mathbf{W}_{i} \cdot \mathbf{M}_{l} d V \\
& =b d_{i} d_{l}\left\{g_{j m} \mathbf{m}_{k} \cdot \mathbf{m}_{n}+g_{j n} \mathbf{m}_{k} \cdot \mathbf{m}_{m}-g_{k m} \mathbf{m}_{j} \cdot \mathbf{m}_{n}-g_{k n} \mathbf{m}_{j} \cdot \mathbf{m}_{m}\right\}
\end{aligned}
$$

$$
\begin{aligned}
T_{(i l)}^{(W F)} & =\int_{V} \mathbf{W}_{i} \cdot \mathbf{F}_{l} d V \\
& =d_{i} \int_{V}\left(\lambda_{j} \nabla \lambda_{k}-\lambda_{k} \nabla \lambda_{j}\right) \cdot \lambda_{m} \lambda_{n} \nabla \lambda_{l} d V \\
& =d_{i} b\left(p_{k l} \int_{A} \lambda_{j} \lambda_{m} \lambda_{n} d A-p_{j l} \int_{A} \lambda_{k} \lambda_{m} \lambda_{n} d A\right)
\end{aligned}
$$

similarly

$$
\begin{aligned}
T_{(i l)}^{(M M)} & =\int_{V} \mathbf{M}_{i} \cdot \mathbf{M}_{l} d V \\
& =b d_{i} d_{l}\left\{g_{j m} \mathbf{m}_{k} \cdot \mathbf{m}_{n}+g_{j n} \mathbf{m}_{k} \cdot \mathbf{m}_{m}+g_{k m} \mathbf{m}_{j} \cdot \mathbf{m}_{n}+g_{n k} \mathbf{m}_{j} \cdot \mathbf{m}_{m}\right\}
\end{aligned}
$$

$$
\begin{aligned}
T_{(i l)}^{(M F)} & =\int_{V} \mathbf{M}_{i} \cdot \mathbf{F}_{l} d V \\
& =d_{i} b\left(p_{k l} \int_{A} \lambda_{j} \lambda_{m} \lambda_{n} d A+p_{j l} \int_{A} \lambda_{k} \lambda_{m} \lambda_{n} d A\right)
\end{aligned}
$$

$$
T_{(i l)}^{(W K)}=0, T_{(i l)}^{(M K)}=0, T_{(i l)}^{(F K)}=0
$$




$$
\begin{aligned}
T_{(i l)}^{(K K)} & =\int_{V} \mathbf{K}_{\mathbf{i}} \cdot \mathbf{K}_{1} d V \\
& =\int_{V} \lambda_{i} \lambda_{l} d V \\
& =\int_{0}^{b} d y \int_{A} \lambda_{i} \lambda_{l} d A \\
& =b \int_{A} \lambda_{i} \lambda_{l} d A \\
T_{(i l)}^{(F F)} & =\int_{V} \mathbf{F}_{\mathbf{i}} \cdot \mathbf{F}_{1} d V \\
& =\int_{V} \lambda_{i} \lambda_{k} \nabla \lambda_{i} \cdot \lambda_{m} \lambda_{n} \nabla \lambda_{l} d V \\
& =c p_{i l} \int_{A} \lambda_{j} \lambda_{k} \lambda_{m} \lambda_{n} d A
\end{aligned}
$$

\subsection{Testing of Finite Element Basis Functions}

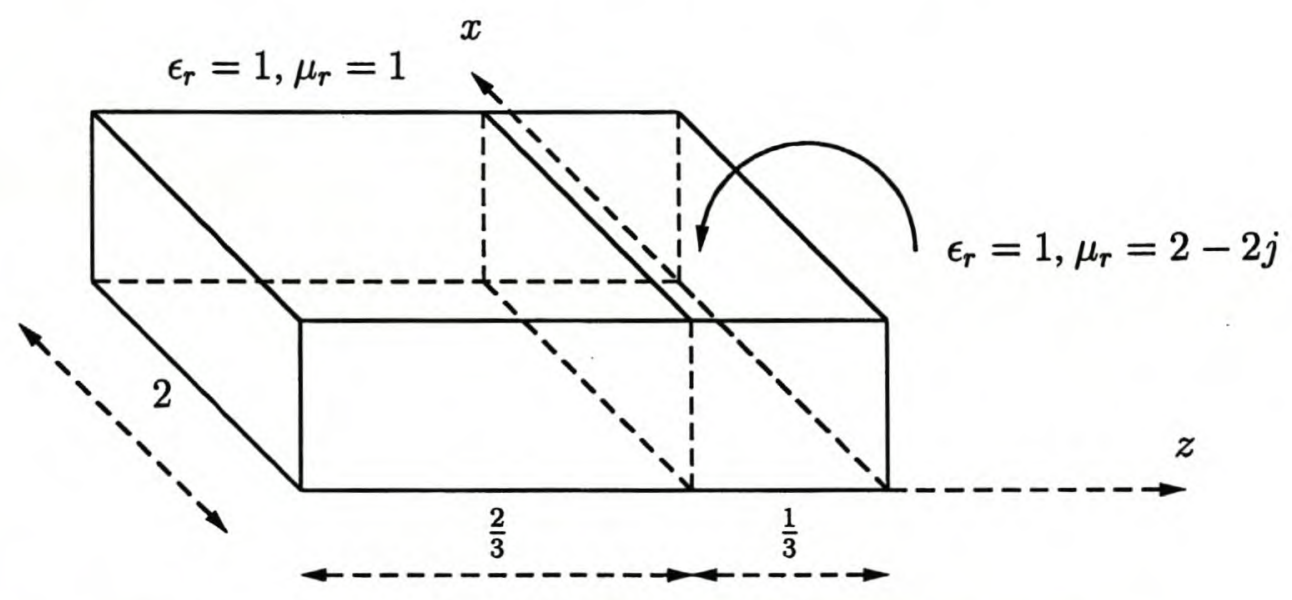

Figure 3.5: Geometry of a test problem with material discontinuities

The basis set appearing in the previous section is a new combination of the standard functions. To ensure that the proposed basis functions are suitable for the modelling intended, the set was tested before incorporation with the surface integral contributions over the surface patch elements. The $\mathrm{x}, \mathrm{z}$ (horizontal) modelling ability is investigated, while it must also be determined whether convergence takes place as the mesh is refined.

The test configuration is the same one as chosen in [43]. For convenience the geometry is also shown in figure 3.5. The test problem is a section of waveguide, with length $1 \mathrm{~m}$, width $2 \mathrm{~m}$ and height $1 \mathrm{~m}$. The axis coordinate system is indicated on figure 3.5 . 


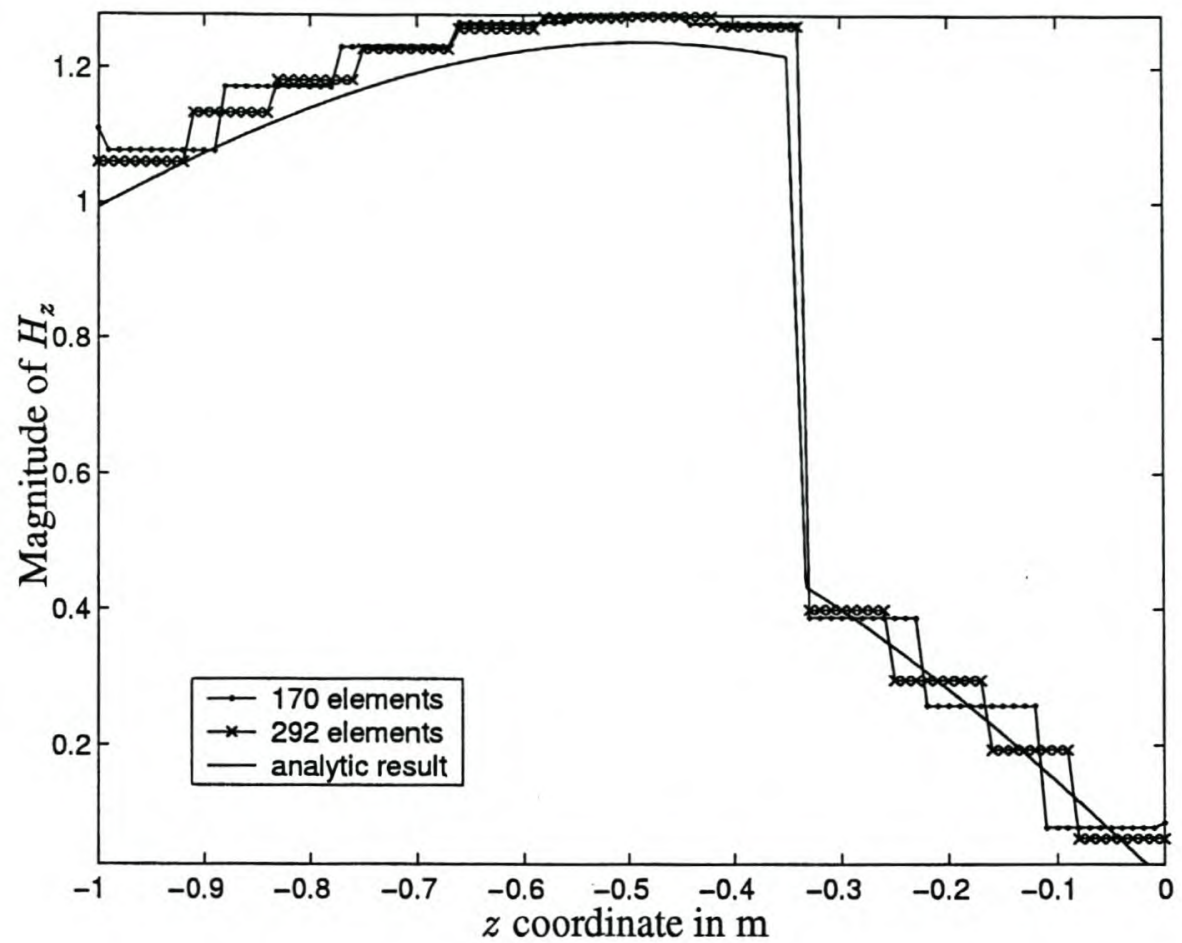

Figure 3.6: The magnetic field $z$ component at $x=0$, obtained via a finite element solution at $8 \mathrm{GHz}$ with a CT/LN basis set. Two mesh sizes were used to demonstrate convergence behaviour.

The waveguide is short-circuited at $\mathrm{z}=0$. The section from $z=0$ to $z=1 / 3$ is filled with a magnetic lossy material, with permeability $\mu_{r}=2-2 j$. A $T E_{10}$ excitation is applied at $\mathrm{z}=1$. The working variable is the volume $H_{z}$ fields.

\subsection{Connection between inner H-fields and tangential E-fields}

\subsubsection{Evaluation of $\left[B_{E}\right]$}

From chapter 2

$$
\left([S]-k_{0}^{2}[T]\right)\left\{\mathcal{H}^{ \pm n}\right\}=j k_{0} Y_{0}\left[B_{E}\right]\left\{\mathcal{E}_{\Gamma_{L}}^{ \pm n}\right\}
$$

that implies that with known tangential electric fields on the boundary $\Gamma_{L}$, magnetic fields inside the volume of the object can be found by applying a finite element solution, where $[S]$ and $[T]$ are the standard finite element matrices. $\left[B_{E}\right]$ is responsible for this connection between the inner and outer parts of the obstacle. 


$$
\begin{aligned}
{\left[B_{E}\right]_{(i l)} } & =\int_{\Gamma_{e l}}\left(\hat{\mathbf{n}}_{L} \times \bar{\tau}_{i}\right) \times \bar{\tau}_{l} d \Gamma \\
& =\hat{\mathbf{n}}_{L} \cdot \int_{\Gamma_{e l}} \bar{\tau}_{i} \times \bar{\tau}_{l} d \Gamma \\
{\left[B_{E}\right]_{(i l)}^{(W W)} } & =\hat{\mathbf{n}}_{L} \cdot \int_{\Gamma_{L}} \mathbf{W}_{i} \times \mathbf{W}_{l} d \Gamma=0 \\
{\left[B_{E}\right]_{(i l)}^{(W K)} } & =\hat{\mathbf{n}}_{L} \cdot \int_{\Gamma_{L}} \mathbf{W}_{i} \times \mathbf{K}_{l} d \Gamma \\
& =d_{i} b \hat{\mathbf{n}}_{L} \cdot\left\{-\mathbf{m}_{k} \int_{u_{1}}^{u_{2}} \lambda_{j} \lambda_{l} d u+\mathbf{m}_{j} \int_{u_{1}}^{u_{2}} \lambda_{k} \lambda_{l} d u\right\} i=1,2,3 \\
{\left[B_{E}\right]_{(i l)}^{(K K)}=} & \hat{\mathbf{n}}_{L} \cdot \int_{\Gamma_{L}} \mathbf{K}_{i} \times \mathbf{K}_{l} d \Gamma=0
\end{aligned}
$$

For the LT/QN basis set, additional to (3.30), (3.31) and (3.32) one requires

$$
\begin{aligned}
{\left[B_{E}\right]_{(i l)}^{(M K)} } & =\hat{\mathbf{n}}_{L} \cdot \int_{\Gamma_{L}} \mathbf{M}_{i} \times \mathbf{K}_{l} d \Gamma \\
& =d_{i} b \hat{\mathbf{n}}_{L} \cdot\left\{\mathbf{m}_{k} \int_{u_{1}}^{u_{2}} \lambda_{j} \lambda_{l} d u+\mathbf{m}_{j} \int_{u_{1}}^{u_{2}} \lambda_{k} \lambda_{l} d u\right\} i=4,5,6
\end{aligned}
$$

where

$$
\begin{array}{r}
\int_{u_{1}}^{u_{2}} \lambda_{i} \lambda_{l} d l \\
=\left\{\begin{array}{cc}
\frac{d_{i}}{3} & i=l=a \text { or } i=l=b \\
\frac{d_{i}}{6} & i=a, l=b \text { or } i=b, l=a \\
0 & \text { otherwise }
\end{array}\right.
\end{array}
$$




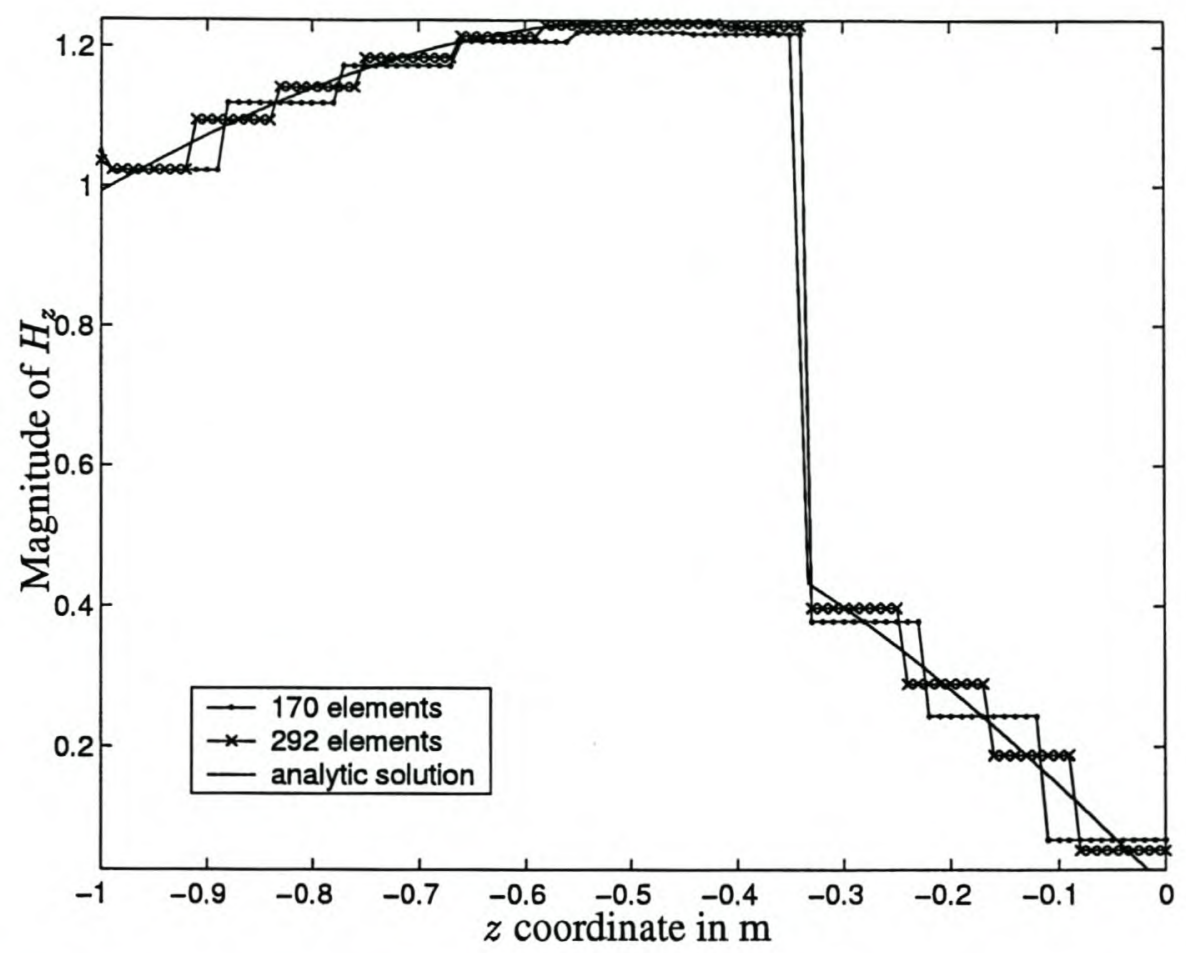

Figure 3.7: Magnitude of $H_{z}$ field at $x=0$, using the connection matrix and Finite Element solution, compared to an analytic solution. Two mesh sizes were used.

\subsection{Testing of Connection between Inner Volume and Outer Surface}

The same problem (figure 3.5) is again used to test the connection between the surface electric fields and the volume magnetic fields. In this case the excitation is the electric field on the surface $z=-1$. Dirichlet boundary conditions are specified on all other external surfaces. The magnetic field magnitude is shown in figure 3.7 along the line $\mathrm{x}=0$.

\subsection{The Waveguide Dyadic Green Functions}

The electric field modal components, subject to the normalization (2.5), are

$$
\begin{aligned}
e_{x}^{(p 0)} & =e_{x}^{p}=0 \\
e_{y}^{(p 0)} & =e_{y}^{p}=-\frac{\sqrt{2}}{\sqrt{a b}} \sin \left(k_{x} x\right) \\
e_{z}^{(p 0)} & =e_{z}^{p}=0
\end{aligned}
$$


The magnetic field modal components, subject to the normalization (2.6), are

$$
\begin{aligned}
& h_{x}^{(p 0)}=h_{x}^{p}=\frac{\sqrt{2}}{\sqrt{a b}} \sin \left(k_{x} x\right) \\
& h_{y}^{(p 0)}=h_{y}^{p}=0 \\
& h_{z}^{(p 0)}=h_{z}^{p}=\frac{\sqrt{2}}{\sqrt{a b}} \frac{k_{x}}{\sqrt{k_{x}^{2}-k_{0}^{2}}} \cos \left(k_{x} x\right)
\end{aligned}
$$

with $k_{x}=\frac{p \pi}{a}$.

The waveguide Green functions with only $T E_{p 0}$ modes considered are

$$
\mathbb{G}_{E}^{ \pm}\left(\mathbf{R}, \mathbf{R}^{\prime}\right)=\sum_{p=1}^{\infty} \frac{1}{a b \gamma_{p 0}} \sin \left(\frac{p \pi}{a} x\right) \sin \left(\frac{p \pi}{a} x^{\prime}\right) e^{\mp\left(z-z^{\prime}\right) \gamma_{p 0}} \hat{\mathbf{y}} \hat{\mathbf{y}}-\frac{1}{k_{0}^{2}} \delta\left(\mathbf{R}-\mathbf{R}^{\prime}\right) \hat{\mathbf{z}} \hat{\mathbf{z}}
$$

and

$$
\begin{aligned}
\mathbb{G}_{H}^{ \pm}\left(\mathbf{R}, \mathbf{R}^{\prime}\right)= & \pm \frac{1}{a b} \sum_{p=1}^{\infty} \sin \left(\frac{p \pi}{a} x\right) \sin \left(\frac{p \pi}{a} x^{\prime}\right) e^{\mp\left(z-z^{\prime}\right) \gamma_{p 0} \hat{\mathbf{x}} \hat{\mathbf{y}}} \\
& +\frac{1}{a b} \sum_{p=1}^{\infty} \frac{p \pi}{a \gamma_{p 0}} \cos \left(\frac{p \pi}{a} x\right) \sin \left(\frac{p \pi}{a} x^{\prime}\right) e^{\mp\left(z-z^{\prime}\right) \gamma_{p 0} \hat{\mathbf{z}} \hat{\mathbf{y}}}
\end{aligned}
$$

with the upper sign corresponding to $z>z^{\prime}$ and the lower sign used when $z<z^{\prime}$. These functions are not defined for $z=z^{\prime}$. Note that discontinuities occur in all components when $z=z^{\prime}$, even though $\mathbf{R} \neq \mathbf{R}^{\prime}$. Practically, the summation is terminated after summing a sufficient number of terms. The number that is sufficient for a specified accuracy, will be examined by numerical experiment in the next chapter.

The function $\mathbb{G}_{H}$ evaluated at points $\mathbf{R}$ and $\mathbf{R}^{\prime}$ a small distance apart cannot be evaluated by direct summation. The convergence of this function under these circumstances is too slow and the required number of modes summed too high to be practical. A numerical integration scheme is implemented to circumvent this difficulty.

\subsection{Integration Scheme}

It was decided to implement a numerical integration scheme to obtain flexibility in the shape of the basis function choice. Each matrix entry to be calculated is the interaction between two rectangular surface patches, with the point $\mathbf{R}$ placed on the first (source patch) and the point $\mathbf{R}^{\prime}$ on the second (observation patch). These points are then integrated over the entire surfaces of the respective patches. The problematic case 
is where the source and observation patches are the same; possibly $\mathbf{R}=\mathbf{R}^{\prime}$. Even so, each integration involving the pair of source and observation patches requires up to three separate integrals to be evaluated. The choice of a numerical scheme allows one to avoid coincident sampling points in the case where the source patch and the observation patch are the same, i.e. avoiding $\mathbf{R}=\mathbf{R}^{\prime}$. This avoids the problem of waveguide discontinuities occurring when $z=z^{\prime}$.

\subsubsection{Gaussian Quadrature}

Gaussian quadrature involves the sampling of the integrand at specified points, $x_{i}$, multiplied with weights, $A_{i}$, specified for each point.

$$
\int_{0}^{1} f(x) d x=\sum_{i=1}^{n} A_{i} f\left(x_{i}\right)
$$

Tables of integrals may be used to determine the sampling points and weights for the order rule required [44]. A transformation to an integral from 0 to 1 is required to use these tables.

\subsubsection{Coordinate Rotation}

The specimen patch is projected onto the $\mathrm{u}-\mathrm{v}$ axis, which forms an angle $\theta$ with the $\mathrm{u}$ axis aligned along the element axis (figure 3.9). The relationship between the specimen element vertices in $(\mathbf{z}, \mathbf{x})$ and $(\mathrm{u}, \mathrm{v})$ coordinates are

$$
\begin{aligned}
& z=\cos \theta u-\sin \theta v \\
& x=\sin \theta u+\cos \theta v
\end{aligned}
$$

and

$$
\begin{aligned}
& u=\cos \theta z+\sin \theta x \\
& v=-\sin \theta z+\cos \theta x
\end{aligned}
$$

The projection of the volume mesh onto the surface of the scatterer results in rectangular surface patches. These have similar properties to the Rao-Wilton-Glisson triangular surface patches described in $[45,33]$. 


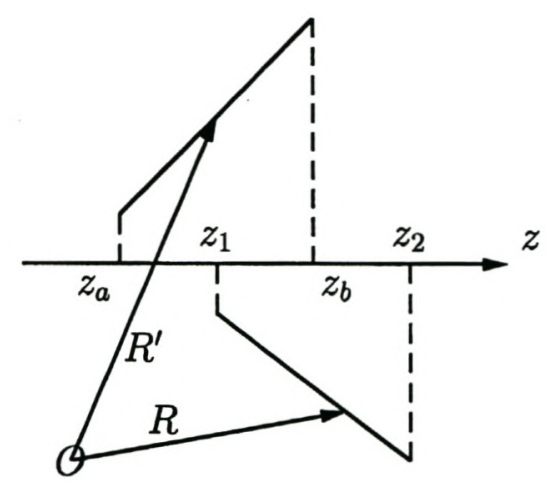

Figure 3.8: Top view of two partially $z$ overlapping surface patches, projected onto the $z$ axis. The integration over $z$ and $z^{\prime}$ is split into three parts, i.e. $\int_{z_{a}}^{z_{1}} \int_{z_{1}}^{z_{2}} \ldots d z d z^{\prime}$, $\int_{z_{1}}^{z_{b}} \int_{z_{1}}^{z_{b}} \ldots d z d z^{\prime}$ and $\int_{z_{1}}^{z_{b}} \int_{z_{b}}^{z_{2}} \ldots d z d z^{\prime}$

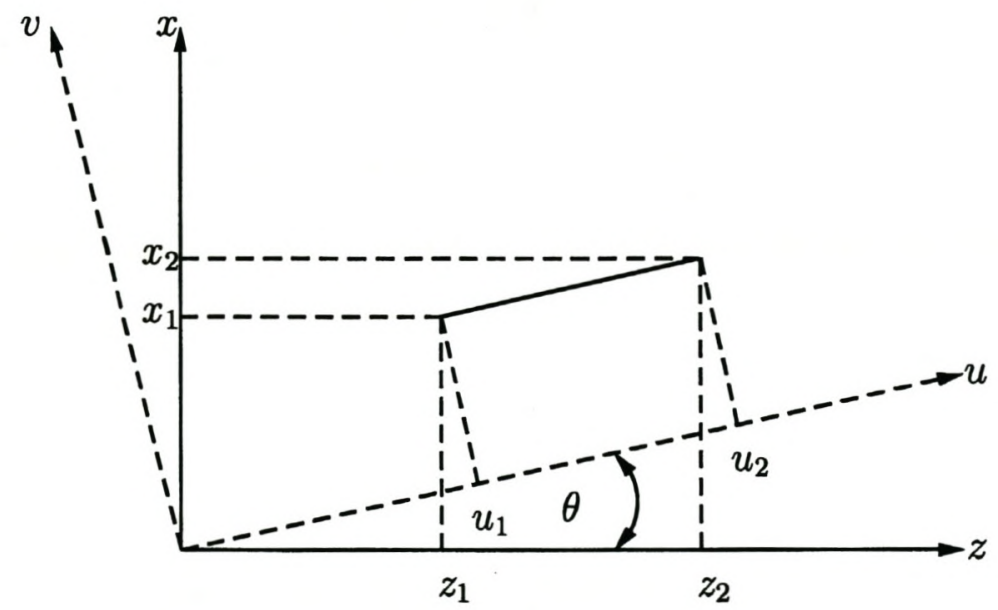

Figure 3.9: Top view of a surface patch: definition of $u v$ axis with respect to $x z$ axis. 


\subsection{Evaluation of $\left[M_{E}\right]$}

$$
\left[M_{E}\right]^{i j}=-2 j k_{0} Z_{0} \int_{\Gamma_{L}} \int_{\Gamma_{L}}\left[\hat{\mathbf{n}}(\mathbf{R}) \times \bar{\tau}_{j}(\mathbf{R})\right] \cdot \mathbb{G}_{E}^{ \pm}\left(\mathbf{R}, \mathbf{R}^{\prime}\right) \cdot\left[\hat{\mathbf{n}}\left(\mathbf{R}^{\prime}\right) \times \bar{\tau}_{i}\left(\mathbf{R}^{\prime}\right)\right] d \Gamma_{L} d \Gamma_{L}^{\prime}
$$

The surface basis functions, $\bar{\tau}_{i}$ and $\bar{\tau}_{j}$ are, at most, linear approximations and ydirected (the $u$-directed basis functions are not involved here). The observation element basis function is

$$
\hat{\mathbf{n}} \times \mathbf{W}_{i}=\left(D_{u} u^{\prime}+D_{k}\right) \hat{\mathbf{y}}^{\prime}=f_{i} \hat{\mathbf{y}}^{\prime} \text { or } \hat{\mathbf{n}} \times \mathbf{M}_{i}=\left(D_{u} u^{\prime}+D_{k}\right) \hat{\mathbf{y}}=f_{i} \hat{\mathbf{y}}^{\prime}
$$

while the source element basis function is

$$
\hat{\mathbf{n}} \times \mathbf{W}_{j}=\left(B_{u} u+B_{k}\right) \hat{\mathbf{y}}=f_{j} \hat{\mathbf{y}} \text { or } \hat{\mathbf{n}} \times \mathbf{M}_{i}=\left(B_{u} u+B_{k}\right) \hat{\mathbf{y}}=f_{j} \hat{\mathbf{y}}
$$

Refer to appendix B for derivations of the tangential surface contributions $D_{y}$ and $D_{k}$ and also $B_{y}$ and $B_{k}$.

Substituting the expression for $\mathbb{G}_{E}^{ \pm}$and the basis functions we have

$$
\begin{aligned}
{\left[M_{E}\right]^{i j}=} & -2 j k_{0} Z_{0} \int_{\Gamma_{L}} \int_{\Gamma_{L}} f_{j} \hat{\mathbf{y}} \cdot \mathbb{G}_{E_{y y}}^{ \pm} \hat{\mathbf{y}} \hat{\mathbf{y}} \cdot f_{i} \hat{\mathbf{y}}^{\prime} d \Gamma_{L} d \Gamma_{L}^{\prime} \\
= & \frac{-2 j k_{0} Z_{0}}{a b} \int_{0}^{b} d y^{\prime} \int_{0}^{b} d y \int_{u_{1}}^{u_{2}}\left(D_{u} u^{\prime}+D_{k}\right) \int_{u_{a}}^{u_{b}}\left(B_{u} u^{\prime}+B_{k}\right) \\
& \quad \sum_{p=1}^{\infty} \frac{1}{\gamma_{p 0}} \sin \left(\frac{p \pi x}{a}\right) \sin \left(\frac{p \pi x^{\prime}}{a}\right) e^{\mp\left(z-z^{\prime}\right) \gamma_{p 0}} d u d u^{\prime}
\end{aligned}
$$

The y-integrations are not subject to transformation and are performed analytically, yielding

$$
\int_{0}^{b} d y=b
$$

as for the observation basis function.

Considering two fully z-overlapping patches, the integration is split into two parts at the discontinuity at $z=z^{\prime}$, treating the discontinuous exponential function as two 
continuous exponentials, also exchanging integration and summation and summing only the first $P$ modes

$$
\begin{gathered}
{\left[M_{E}\right]^{i j}=-2 j k_{0} Z_{0} \frac{b}{a} \sum_{p=1}^{P} \frac{1}{\gamma_{p 0}} \int_{u_{a}}^{u_{b}}\left(D_{u} u^{\prime}+D_{k}\right) \sin \left(\frac{p \pi x}{a}\right) \cdot} \\
\left\{\int_{u_{1}}^{u_{c}}\left(B_{u} u^{\prime}+B_{k}\right) \sin \left(\frac{p \pi x}{a}\right) e^{s \gamma_{p 0}\left(z-z^{\prime}\right)} d u+\int_{u_{c}}^{u_{2}}\left(B_{u} u^{\prime}+B_{k}\right) \sin \left(\frac{p \pi x}{a}\right) e^{-s \gamma_{p 0}\left(z-z^{\prime}\right)} d u\right\} d u^{\prime}
\end{gathered}
$$

with $u_{c}=z^{\prime} \cos \left(\theta_{j}\right)+x_{c} \sin \left(\theta_{j}\right), x_{c}=\frac{x_{2}-x_{1}}{z_{2}-z_{1}}\left(z^{\prime}-z_{1}\right)+x_{1}$ and

$$
s=\frac{\hat{\mathbf{u}}_{j} \cdot \hat{\mathbf{z}}}{\left|\hat{\mathbf{u}}_{j} \cdot \hat{\mathbf{z}}\right|}
$$

bearing in mind that integration over the surface patches is always performed in an anticlockwise direction. The choice of $s$ is explained in figures 3.10 and 3.11; it depends on the direction of integration of the source patch with respect to the $\mathrm{z}$ axis.

The integral over the u-axis is performed numerically. For Gaussian integration it is required that the integration limits are from 0 to 1 . Let

$$
\begin{aligned}
m & =\frac{u-u_{1}}{u_{c}-u_{1}} \\
k & =\frac{u-u_{c}}{u_{2}-u_{c}} \\
n & =\frac{u^{\prime}-u_{a}}{u_{b}-u_{a}}
\end{aligned}
$$

then

$$
\begin{aligned}
{\left[M_{E}\right]^{i j}=} & -\left(u_{b}-u_{a}\right) \frac{2 j k_{0} Z_{0} b}{a} \sum_{p=1}^{P} \int_{0}^{1}\left(D_{u} u_{i}+D_{k}\right) \sin \left(k_{x} x_{i}\right) \\
& \left\{\left(u_{c}-u_{1}\right) \int_{0}^{1}\left(B_{u} u_{j_{1}}+B_{k}\right) \sin \left(k_{x} x_{j_{1}}\right) e^{s \gamma_{p 0}\left(z_{i}-z_{j_{1}}\right)} d m\right. \\
+ & \left.\left(u_{2}-u_{c}\right) \int_{0}^{1}\left(B_{u} u_{j_{2}}+B_{k}\right) \sin \left(k_{x} x_{j_{2}}\right) e^{-s \gamma_{p 0}\left(z_{i}-z_{j_{2}}\right)} d k\right\} d n
\end{aligned}
$$

with $x_{i}=\sin \theta_{i} u_{i}+\cos \theta_{i} v_{i}$ and $z_{i}=\cos \theta_{i} u_{i}-\sin \theta_{i} v_{i}$, using the transformations 


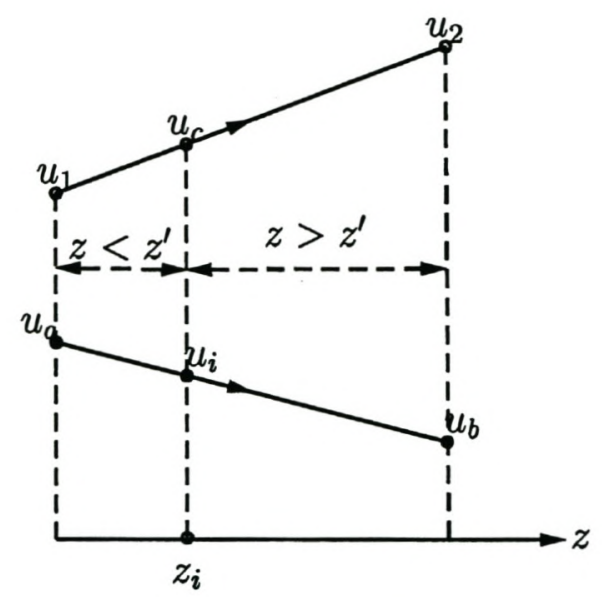

Figure 3.10: Top view of two surface patches: the use of the parameter sfor a source patch integrated in a positive z-direction. For this case $s=1$.

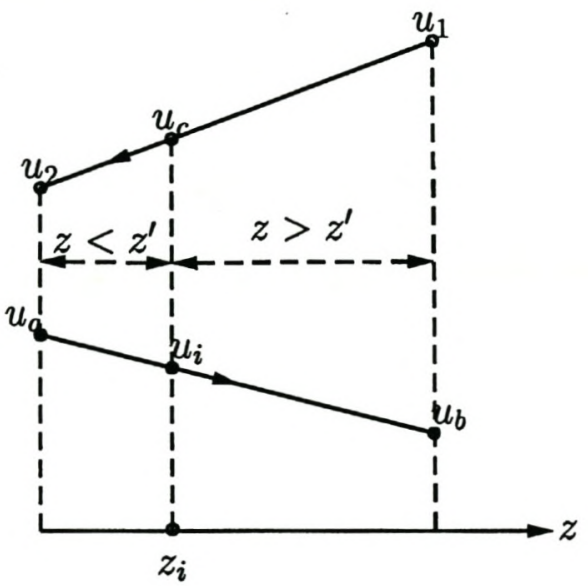

Figure 3.11: Top view of two surface patches: the use of the parameter sfor source patch integrated in a negative $z$-direction. For this case $s=-1$. 
(3.40) and (3.41) and

$$
\begin{aligned}
u_{i} & =n\left(u_{b}-u_{a}\right)+u_{a} \\
u_{j_{1}} & =m\left(u_{c}-u_{1}\right)+u_{1} \\
u_{j_{2}} & =k\left(u_{2}-u_{i}\right)+u_{c} \\
x_{j_{1}} & =\sin \theta_{j} u_{j_{1}}+\cos \theta_{j} v_{j} \\
x_{j_{2}} & =\sin \theta_{j} u_{j_{2}}+\cos \theta_{j} v_{j} \\
g_{i j_{1}} & =z_{i}-z_{j_{1}}=\cos \theta_{i} u_{i}-\sin \theta_{i} v_{i}-\left(\cos \theta_{j} u_{j_{2}}+\sin \theta_{j} v_{j}\right) \\
g_{i j_{2}} & =z_{i}-z_{j_{2}}=\cos \theta_{i}-\sin \theta_{i} v_{i}-\left(\cos \theta_{j} u_{j_{2}}+\sin \theta_{j} v_{j}\right)
\end{aligned}
$$

Using the non-uniformly spaced sampling points for Gaussian integration and the associated weights (3.39)

$$
\begin{aligned}
{\left[M_{E}\right]^{i j}=} & -2\left(u_{b}-u_{a}\right) \frac{j k_{0} Z_{0} b}{a} \sum_{p=1}^{P} \sum_{i} A_{i}\left(D_{u} u_{i}+D_{k}\right) \sin \left(k_{x} x_{i}\right) \\
& \left\{\left(u_{c}-u_{1}\right) \sum_{j_{1}} A_{j_{1}}\left(B_{u} u_{j_{1}}+B_{k}\right) \sin \left(k_{x} x_{j_{1}}\right) e^{s \gamma_{p 0}\left(z_{i}-z_{j_{1}}\right)}\right. \\
+ & \left.\left(u_{2}-u_{c}\right) \sum_{j_{2}} A_{j_{2}}\left(B_{u} u_{j_{2}}+B_{k}\right) \sin \left(k_{x} x_{j_{2}}\right) e^{-s \gamma_{p 0}\left(z_{i}-z_{j_{2}}\right)}\right\}
\end{aligned}
$$

\subsection{Evaluation of $\left[M_{H}\right]$}

The matrix entry for the interaction between a basis function $i$ and a basis function $j$ is

$$
\left.\left[M_{H}\right]^{i j}=2 \int_{\Gamma_{L}} \int_{\Gamma_{L}}\left[\hat{\mathbf{n}}_{L}(\mathbf{R}) \times \bar{\tau}_{j}(\mathbf{R})\right] \cdot \mathbb{G}_{H}^{ \pm}\left(\mathbf{R}, \mathbf{R}^{\prime}\right) \cdot\left[\hat{\mathbf{n}}_{L}\left(\mathbf{R}^{\prime}\right)\right) \times \bar{\tau}_{i}\left(\mathbf{R}^{\prime}\right)\right] d \Gamma_{L} d \Gamma_{L}^{\prime}
$$

In evaluating the entries of $\left[M_{H}\right]$, the interactions are between basis functions $\mathbf{W}_{i}$ and $\mathbf{K}_{j}$ and also between $\mathbf{M}_{i}$ and $\mathbf{K}_{j}$. The surface basis functions are, at most, linear approximations (refer to appendix B), depending on the order of the basis set implemented:

$$
\begin{aligned}
\hat{\mathbf{n}} \times \mathbf{W}_{i} & =\left(D_{u} u^{\prime}+D_{k}\right) \hat{\mathbf{y}}=f_{i} \hat{\mathbf{y}}^{\prime} \text { or } \hat{\mathbf{n}} \times \mathbf{M}_{i}=\left(D_{u} u^{\prime}+D_{k}\right) \hat{\mathbf{y}}=f_{i} \hat{\mathbf{y}}^{\prime} \\
\hat{\mathbf{n}} \times \mathbf{K}_{j} & =\left(B_{u} u+B_{k}\right) \hat{\mathbf{u}}=f_{j} \hat{\mathbf{u}}
\end{aligned}
$$




$$
\begin{aligned}
{\left[M_{H}\right]^{i j} } & =2 \int_{\Gamma_{L}} \int_{\Gamma_{L}} f_{j} \hat{\mathbf{u}}_{j} \cdot\left(G_{H_{x y}} \hat{\mathbf{x}} \hat{\mathbf{y}}^{\prime}+G_{H_{z y}} \hat{\mathbf{z}} \hat{\mathbf{y}}^{\prime}\right) \cdot f_{i} \hat{\mathbf{y}}^{\prime} d \Gamma d \Gamma^{\prime} \\
& =\frac{2}{a b} \int_{0}^{b} d y^{\prime} \int_{0}^{b} d y \int_{u_{a}}^{u_{b}}\left(D_{u} u^{\prime}+D_{k}\right) \\
& \cdot\left(\int_{u_{1}}^{u_{2}}\left(B_{u} u+B_{k}\right)\left(\hat{\mathbf{u}}_{j} \cdot \hat{\mathbf{x}}\right) \sum_{p=1}^{\infty} \pm \sin \left(\frac{p \pi x}{a}\right) \sin \left(\frac{p \pi x^{\prime}}{a}\right) e^{\mp\left(z-z^{\prime}\right) \gamma_{p 0}} d u\right. \\
& \left.+\int_{u_{1}}^{u_{2}}\left(B_{u} u+B_{k}\right)\left(\hat{\mathbf{u}}_{j} \cdot \hat{\mathbf{z}}\right) \sum_{p=1}^{\infty} \frac{p \pi}{a \gamma_{p 0}} \cos \left(\frac{p \pi x}{a}\right) \sin \left(\frac{p \pi x^{\prime}}{a}\right) e^{\mp\left(z-z^{\prime}\right) \gamma_{p 0}} d u\right) d u^{\prime}
\end{aligned}
$$

Now, swapping integration and summation, and terminating the summation after $P$ terms, we have

$$
\begin{aligned}
{\left[M_{H}\right]^{i j}=} & \frac{2 b}{a} \sum_{p=1}^{P} \int_{u_{a}}^{u_{b}}\left(D_{u} u^{\prime}+D_{k}\right) \\
& \left\{ \pm \int_{u_{1}}^{u_{2}}\left(B_{u} u+B_{k}\right)\left(\hat{\mathbf{u}}_{j} \cdot \hat{\mathbf{x}}\right) \sin \left(\frac{p \pi x}{a}\right) \sin \left(\frac{p \pi x^{\prime}}{a}\right) e^{\mp\left(z-z^{\prime}\right) \gamma_{p 0}} d u\right. \\
+ & \left.\frac{p \pi}{a \gamma_{p 0}} \int_{u_{1}}^{u_{2}}\left(B_{u} u+B_{k}\right)\left(\hat{\mathbf{u}}_{j} \cdot \hat{\mathbf{z}}\right) \cos \left(\frac{p \pi x}{a}\right) \sin \left(\frac{p \pi x^{\prime}}{a}\right) e^{\mp\left(z-z^{\prime}\right) \gamma_{p 0}} d u\right\} d u^{\prime}
\end{aligned}
$$

The integration depends on the overlap between the z-coordinates of the two patches, as shown in figure 3.8 .

The double integral in (3.53) is always split into overlapping and non-overlapping integrations; every partially or fully overlapping double integral has the following con- 
tribution (fully overlapping section):

$$
\begin{aligned}
{\left[M_{H}\right]^{i j}=} & \frac{2 b}{a} \sum_{p=1}^{P}\left\{\int_{u_{a}}^{u_{b}}\left(\hat{\mathbf{u}}_{j} \cdot \hat{\mathbf{x}}\right)\left(D_{u} u^{\prime}+D_{k}\right) \sin \left(\frac{p \pi x^{\prime}}{a}\right)\right. \\
& \cdot\left[\int_{u_{1}}^{u_{c}}-s\left(B_{u} u+B_{k}\right) \sin \left(\frac{p \pi x}{a}\right) e^{s \gamma_{p 0}\left(z-z^{\prime}\right)} d u\right. \\
& \left.+\int_{u_{c}}^{u_{2}} s\left(B_{u} u+B_{k}\right) \sin \left(\frac{p \pi x}{a}\right) e^{-s \gamma_{p 0}\left(z-z^{\prime}\right)} d u\right] d u^{\prime} \\
& +\frac{p \pi}{a \gamma_{p 0}} \int_{u_{a}}^{u_{b}}\left(\hat{\mathbf{u}}_{j} \cdot \hat{\mathbf{z}}\right)\left(D_{u} u^{\prime}+D_{k}\right) \sin \left(\frac{p \pi x^{\prime}}{a}\right) \\
& \cdot\left[\int_{u_{1}}^{u_{c}}\left(B_{u} u+B_{k}\right) \cos \left(\frac{p \pi x}{a}\right) e^{s \gamma_{p 0}\left(z-z^{\prime}\right)} d u\right. \\
& \left.\left.+\int_{u_{c}}^{u_{2}}\left(B_{u} u+B_{k}\right) \cos \left(\frac{p \pi x}{a}\right) e^{-s \gamma_{p 0}\left(z-z^{\prime}\right)} d u\right] d u^{\prime}\right\}
\end{aligned}
$$

The transformed expression is then

$$
\begin{aligned}
{\left[M_{H}\right]^{i j}=} & \frac{2 b}{a}\left(u_{b}-u_{a}\right) \sum_{p=1}^{P}\left\{\int_{0}^{1}\left(\hat{\mathbf{u}}_{j} \cdot \hat{\mathbf{x}}\right)\left(D_{u} u_{i}+D_{k}\right) \sin \left(\frac{p \pi x_{i}}{a}\right)\right. \\
& \cdot\left[\int_{0}^{1}-s\left(u_{c}-u_{1}\right)\left(B_{u} u_{j_{1}}+B_{k}\right) \sin \left(\frac{p \pi x_{j_{1}}}{a}\right) e^{s \gamma_{p 0}\left(z_{j_{1}}-z_{i}\right)} d m\right. \\
+ & \left.\int_{0}^{1} s\left(u_{2}-u_{c}\right)\left(B_{u} u_{j_{2}}+B_{k}\right) \sin \left(\frac{p \pi x_{j_{2}}}{a}\right) e^{-s \gamma_{p 0}\left(z_{j_{2}}-z_{i}\right)} d k\right] d n \\
+ & \frac{p \pi}{a \gamma_{p 0}} \int_{0}^{1}\left(\hat{\mathbf{u}}_{j} \cdot \hat{\mathbf{z}}\right)\left(D_{u} u_{i}+D_{k}\right) \sin \left(\frac{p \pi x_{i}}{a}\right) \\
& \cdot\left[\int_{0}^{1}\left(u_{c}-u_{1}\right)\left(B_{u} u_{j_{1}}+B_{k}\right) \cos \left(\frac{p \pi x_{j_{1}}}{a}\right) e^{s \gamma_{p 0}\left(z_{j_{1}}-z_{i}\right)} d m\right. \\
& \left.+\int_{0}^{1}\left(u_{2}-u_{c}\right)\left(B_{u} u_{j_{2}}+B_{k}\right) \cos \left(\frac{p \pi x_{j_{2}}}{a}\right) e^{-s \gamma_{p 0}\left(z_{j_{2}}-z_{i}\right)} d k\right] d n(\hat{\jmath} .58)
\end{aligned}
$$

with $x_{i}=\sin \theta_{i} u_{i}+\cos \theta_{i} v_{i}$ and $z_{i}=\cos \theta_{i} u_{i}-\sin \theta_{i} v_{i}$, once again using the coordinate transformations (3.40) and (3.41). The integration limit transformations are given in section 3.9 in (3.49). 


$$
\begin{aligned}
{\left[M_{H}\right]^{i j}=} & \frac{2 b}{a}\left(u_{b}-u_{a}\right) \sum_{p=1}^{P}\left\{\sum_{i} A_{i}\left(\hat{\mathbf{u}}_{j} \cdot \hat{\mathbf{x}}\right)\left(D_{u} u_{i}+D_{k}\right) \sin \left(\frac{p \pi x_{i}}{a}\right)\right. \\
& \cdot\left[\sum_{j_{1}} A_{j_{1}}(-s)\left(u_{c}-u_{1}\right)\left(B_{u} u_{j_{1}}+B_{k}\right) \sin \left(\frac{p \pi x_{j_{1}}}{a}\right) e^{s \gamma_{p 0}\left(z_{j_{1}}-z_{i}\right)}\right. \\
+ & \left.\sum_{j_{2}} A_{j_{2}} s\left(u_{2}-u_{c}\right)\left(B_{u} u_{j_{2}}+B_{k}\right) \sin \left(\frac{p \pi x_{j_{2}}}{a}\right) e^{-s \gamma_{p 0}\left(z_{j_{2}}-z_{i}\right)}\right] \\
+ & \frac{p \pi}{a \gamma_{p 0}} \sum_{i} A_{i}\left(\hat{\mathbf{u}}_{j} \cdot \hat{\mathbf{z}}\right)\left(D_{u} u_{i}+D_{k}\right) \sin \left(\frac{p \pi x_{i}}{a}\right) \\
& \cdot\left[\sum_{j_{1}} A_{j_{1}}\left(u_{c}-u_{1}\right)\left(B_{u} u_{j_{1}}+B_{k}\right) \cos \left(\frac{p \pi x_{j_{1}}}{a}\right) e^{s \gamma_{p 0}\left(z_{j_{1}}-z_{i}\right)}\right. \\
+ & \left.\left.\sum_{j_{2}} A_{j_{2}}\left(u_{2}-u_{c}\right)\left(B_{u} u_{j_{2}}+B_{k}\right) \cos \left(\frac{p \pi x_{j_{2}}}{a}\right) e^{-s \gamma_{p 0}\left(z_{j_{2}}-z_{i}\right)}\right]\right\}
\end{aligned}
$$

Integrals for the other two cases indicated in figure 3.8 are simpler, since these involve either $z<z^{\prime}$ or $z>z^{\prime}$ and not both, as in the case of overlapping patch $\mathrm{z}$-coordinates.

\subsection{Solution of Matrix Equations}

In chapter 2 we had two linked matrix equations representing a system of linear equations to be solved for the tangential electric field coefficients $\left\{\mathcal{E}_{\Gamma_{L}}^{ \pm n}\right\}$ and the magnetic field coefficients $\left\{\mathcal{H}^{ \pm n}\right\}$, which represent both the magnetic fields inside the post and the tangential surface magnetic fields

$$
\begin{aligned}
& {\left[B_{E}\right]\left\{\mathcal{E}_{\Gamma_{L}}^{ \pm n}\right\}=\left\{\mathbf{e}^{ \pm n}\right\}+\left[M_{E}\right]\left\{\mathcal{H}_{\Gamma_{L}}^{ \pm n}\right\}+\left[M_{H}\right]\left\{\mathcal{E}_{\Gamma_{L}}^{ \pm n}\right\}} \\
& {[F]\left\{\mathcal{H}^{ \pm n}\right\}=j k_{0} Y_{0}\left[B_{E}\right]\left\{\mathcal{E}_{\Gamma_{L}}^{ \pm n}\right\}}
\end{aligned}
$$

with $[F]=[S]-k_{0}^{2}[T]$.

The structure of $\left[B_{E}\right]$ and $\left[M_{H}\right]$ are such that these are not invertible (figure 3.12). When solving the matrix equations, any operation involving the inverses of either matrix or combinations of these, should be avoided. This matrix fill pattern is a result of the quasi-2D nature of the problems considered here. If a tetrahedral finite element discretization is employed for a general 3D object as an extension to this implementation, many of the empty blocks in the matrices will fill in with entries. 
It is interesting to note that for the specific geometries considered, the formulation is no longer a Galerkin technique, which is also reflected in the non-symmetry of the $\left[M_{H}\right]$ matrix entries. Although Galerkin weighting is very popular, with point matching also being used often, it is by no means required [46] to use only these type of weighting functions. With some of the restrictions on the geometry introduced in section 3.2 lifted, the weight functions would once again be contained in the same set as the basis functions.

\subsubsection{Perfect Electric Conducting (PEC) Posts}

On the surface of the metallic post, all tangential E-fields are zero, while all internal fields are also zero. Only one equation remains for the surface magnetic fields

$$
\left[\mathcal{M}_{E}\right]\left\{\mathcal{H}_{\Gamma_{L}}^{ \pm n}\right\}=-\left\{e^{ \pm n}\right\}
$$

The finite element discretization is not required and only the surface patch integrals are performed [47].

\subsubsection{Perfect Magnetic Conducting (PMC) Posts}

Although perfect magnetic posts in waveguide do not represent any physical configuration, these are useful for validating parts of the formulation separately. Perfectly magnetic conductors are used to represent symmetry planes in numerical codes and are applied naturally on all boundary surfaces without specified boundary conditions in FE codes.

The surface matrix equation, (3.61), reduces to

$$
\left(\left[\mathcal{M}_{H}\right]-\left[B_{E}\right]\right)\left\{\mathcal{H}_{\Gamma_{L}}^{ \pm n}\right\}=-\left\{e^{ \pm n}\right\}
$$

\subsubsection{Dielectric Posts}

Solving for the electric tangential fields first requires the inverse of the finite element matrix $[F]$, which is invertible and avoids the inverse of $\left[B_{E}\right]$

$$
\begin{aligned}
& \left\{\left[B_{E}\right]-\left[M_{H}\right]-j k_{0} Y_{0}\left[M_{E}\right][F]^{-1}\left[B_{E}\right]\right\}\left\{\mathcal{E}_{\Gamma_{L}}^{ \pm n}\right\}=\left\{e^{ \pm n}\right\} \\
& \left\{\mathcal{H}^{ \pm n}\right\}=j k_{0} Y_{0}[F]^{-1}\left[B_{E}\right]\left\{\mathcal{E}_{\Gamma_{L}}^{ \pm n}\right\}
\end{aligned}
$$


For equation (3.60), empty (zero entries only) rows and columns are removed from the matrices $\left[B_{E}\right],\left[M_{H}\right]$ and $\left[M_{E}\right]$, to obtain compressed matrices as shown in row two of figure 3.12. A matrix equation for the surface electric fields results, with dimension $M \times K$, with $M=K$ for the CT/LN basis set. After the E-coefficients are calculated, the full set of magnetic field coefficients may be determined by using the non-compressed forms of these matrices in equation (3.61).

In the case of the LT/QN basis set with specifically the single element discretization in the $y$ direction, the surface matrix equation produces an unexpected difficulty; that is $2 M=K$, in other words an over specified system of equations for the surface electric fields results. To solve this problem, one would either have to increase the order of the $\mathrm{y}$-modelling basis functions to second order, or move on to a more general problem, such as partial height posts for example. When more than one level of elements are meshed in the $y$-direction, with the associated inclusion of the full set of Green function dyadic components, the LT/QN basis set suggested in (3.3) will be suitable for a higher order approximation as is. For the results in chapter 4, only the CT/LN basis set was implemented. The requirement that the number of surface electric field degrees of freedom is equal to the number of magnetic field degrees of freedom is automatically satisfied when a full 3D tetrahedral mesh and the full set of Green function dyadic components are implemented.

\subsection{Conclusion}

With the matrices set up and the two coupled equations solved, the next chapter proceeds to examine the results obtained with this technique. To determine the accuracy of the solutions one has to choose the mesh discretization, in conjunction with the number of waveguide modes summed. One would like to obtain the best possible solution for S-parameters while summing as few modes as possible. Also, the number of integration points per surface patch should be such that the numerical integrals are converged.

A higher order finite element discretization in the post should allow one to sum more modes accurately, while the magnetic fields inside the post are still accurate representations of the scattered magnetic field inside the scatterer. For a finite element discretization that approximates some components of the magnetic field inside the post as constants, one would expect that not too many modes could be summed before the finite element mesh becomes a restricting factor. These ideas will be examined in the following chapters by considering a number of carefully chosen examples. 


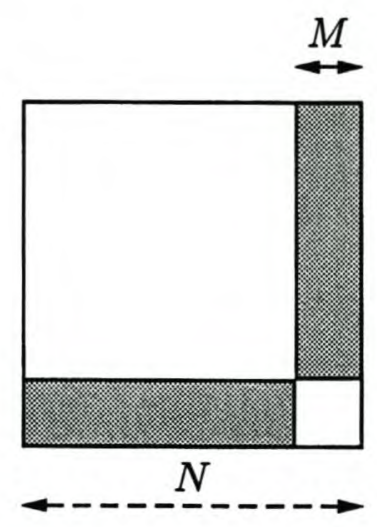

$\left[B_{E}\right]$

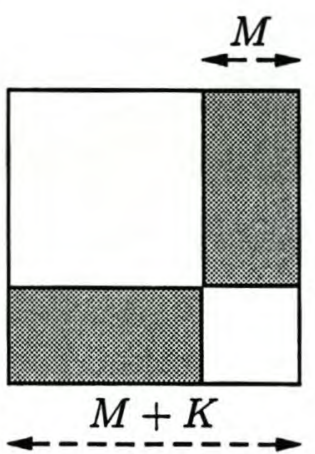

$\left[B_{E}\right]$

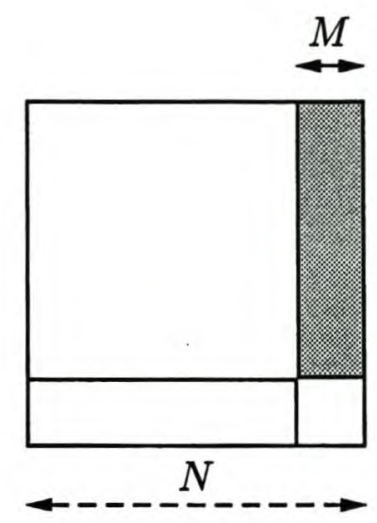

$\left[M_{H}\right]$

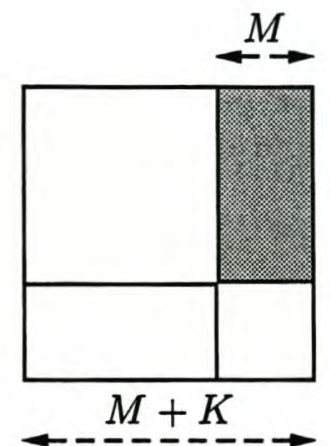

$\left[M_{H}\right]$

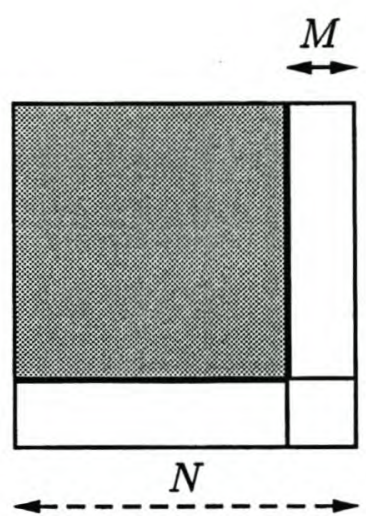

$\left[M_{E}\right]$

Figure 3.12: Position of possible entries of the non-finite element matrices of equations (3.60) and (3.61). $N$ is the number of total degrees of freedom and includes both magnetic and electric coefficients, $M$ is the number of electric field unknowns and $K$ is the number of surface tangential magnetic field unknowns. The second row shows these matrices in compressed format (involving only surface coefficients) as used in equation (3.60). $K=M$ when the lowest order basis set is used. 


\section{Chapter 4}

\section{Results for Posts in Waveguide}

\subsection{Introduction}

The Huygens formulation has been implemented in MATLAB ${ }^{\circledR}$ to solve scattering problems for posts extending from the bottom to the top of the waveguide. Obtaining accurate $S$-parameter results requires the choice of a suitably fine mesh, sufficient waveguide modes and an adequate number of integration points per surface patch element. The accuracy of the numerical results is determined by the number of volume elements and surface patches and corresponding basis function order of approximation, the number of waveguide modes that are considered and the convergence of the numerical integration scheme. An adequate choice of these parameters yields results corresponding to those obtained with a commercial numerical code.

In this chapter, $S$ parameter results for dielectric, PEC and PMC posts with a circular cross-section in an X-band rectangular waveguide are presented through the use of the Huygens formulation.

A commercial finite volume code, CST Microwave Studio ${ }^{\circledR}$, was used in most cases to obtain $S$ parameter data for comparative purposes. In the case of perfectly magnetic posts, which corresponds to a natural boundary condition, ANSYS ${ }^{\circledR}$, a commercial finite element code was used to verify the results, since it was the only commercial package that could apply natural boundary conditions on an arbitrary surface not on the outer boundary of the problem.

The length of the waveguide in CST Microwave Studio ${ }^{\circledR}$ was always $30 \mathrm{~mm}$, with the post or multiple post configuration placed in the middle of the length of waveguide. In the Huygens code $l_{1}=-15 \mathrm{~mm}$ and $l_{2}=15 \mathrm{~mm}$ with the post centre at $z=0$. This corresponds to the geometric setup in CST Microwave Studio ${ }^{\circledR}$ and ANSYS ${ }^{\circledR}$. 


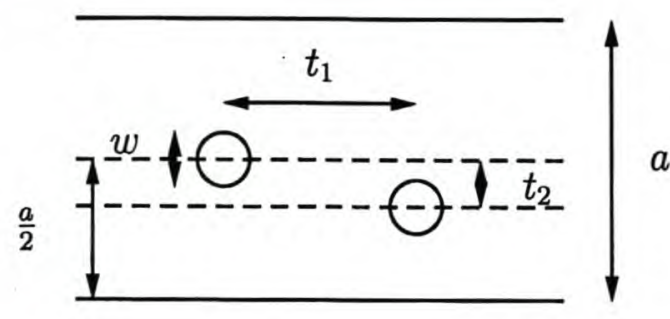

Figure 4.1: two posts, no symmetry

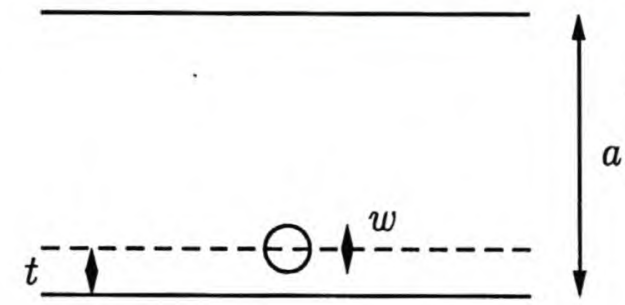

Figure 4.3: off-centre thin post

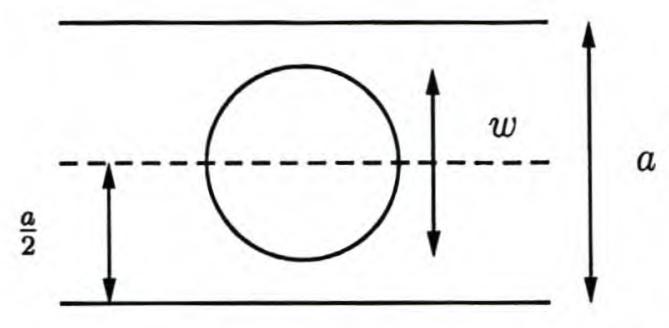

Figure 4.2: large centre post, symmetric

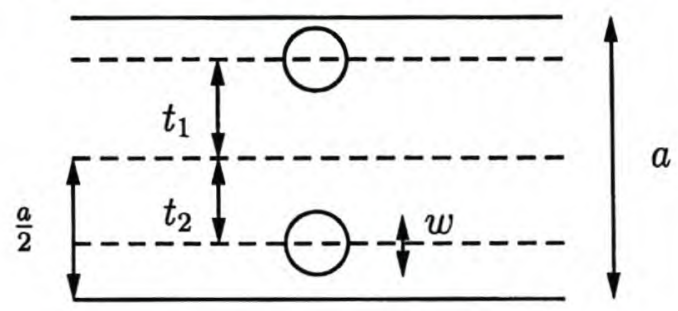

Figure 4.4: two posts in non symmetric configuration

For centre posts or other symmetrical configurations around $x=a / 2$ a simplification can be made: only even modes need to be considered in the analysis. These are modes that have a symmetrical field distribution around $x=a / 2$, i.e. $T E_{p 0}$ modes with odd indices, viz. $p=1,3,5, \ldots$.

A number of test problems are carefully chosen to determine that all parts of the implementation are working correctly. Following the progression of test problems, the Huygens formulation and implementation can be verified step by step. Choosing a single problem configuration only can be misleading, therefore a number of examples are required. For example, in many small central dielectric or magnetic post cases, the first term in the surface integral of (2.8) dominates the second term, so that an error in the latter would not be immediately obvious. With the selection of problems chosen below, one can verify all parts of the implementation. In figures 4.1 to 4.4 the selected configurations are illustrated.

\subsection{Perfectly Electric Conducting (PEC) Posts}

The simplest test cases are perfectly conducting posts. Here tangential electric fields are zero on a PEC surface. Since the volume fields are also zero, only the surface magnetic fields are solved. The second term of the surface integral in (2.8) reduces to zero (see (3.62)).

Two perfectly conducting posts were considered in the configuration of figure 4.4. The 


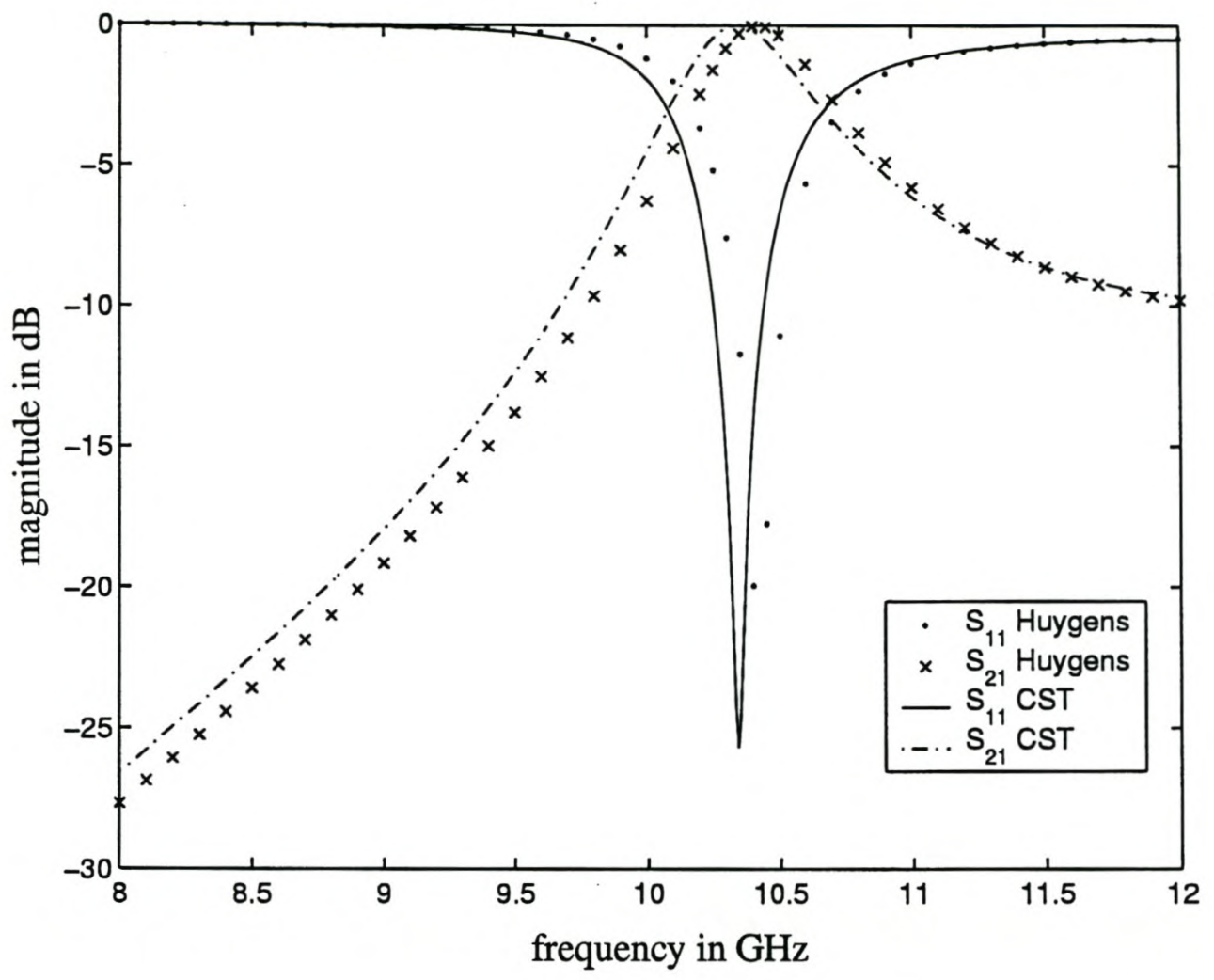

Figure 4.5: $S_{11}$ and $S_{21}$ magnitude for two PEC posts

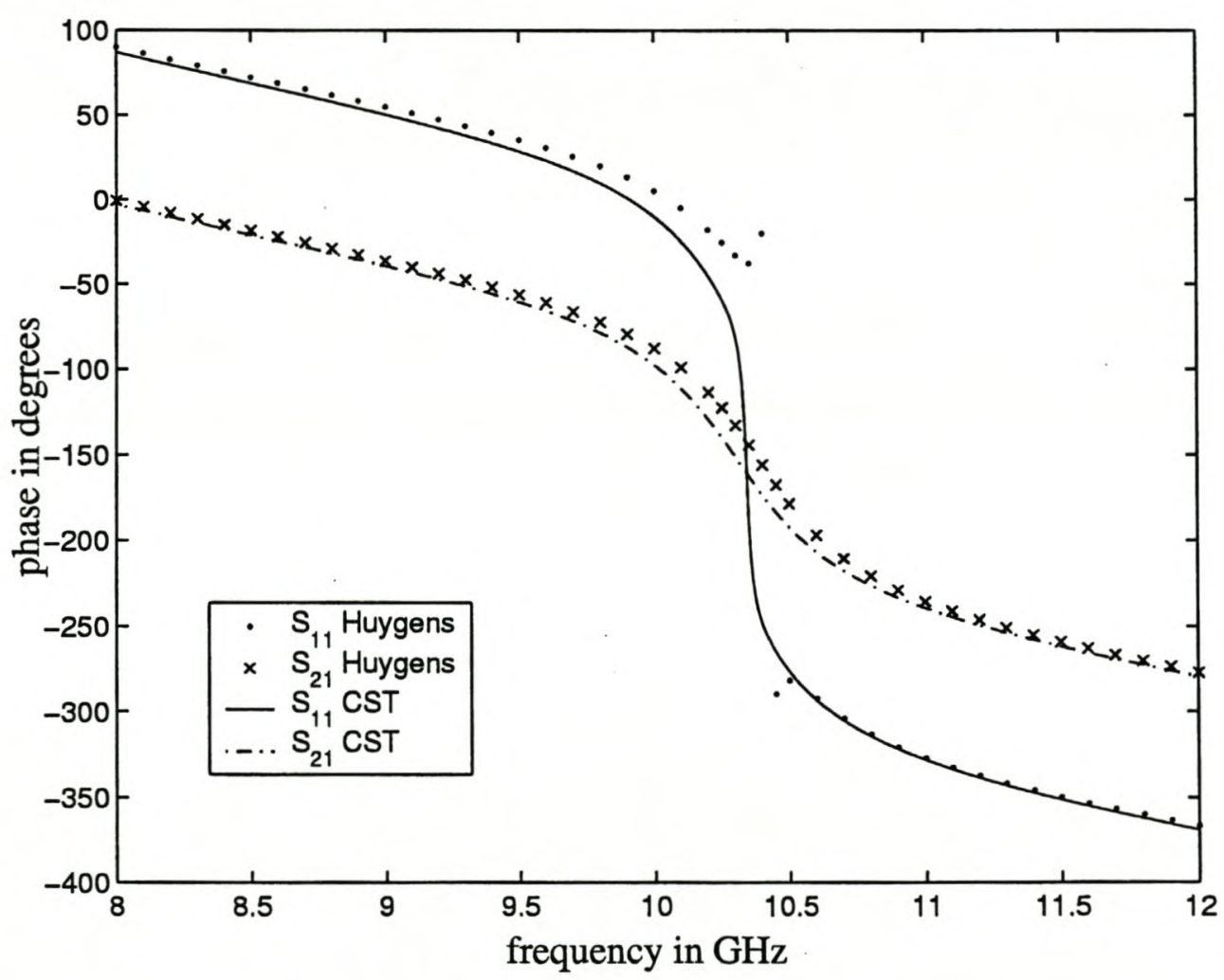

Figure 4.6: $S_{11}$ and $S_{21}$ phase for two PEC posts 
parameters were $t_{1}=16 \mathrm{~mm}, w=2 \mathrm{~mm}$ and $t_{2}=1 \mathrm{~mm}$. The $S$ parameter results appear in figures 4.5 and 4.6. A frequency shift of less than $1 \%$ is visible at resonance.

\subsection{Perfect Magnetic Conducting (PMC) Posts}

This test eliminates the first term of the surface integral in (2.8), while relying on the second term and the connection of the internal magnetic and external electric fields. The volume elements again reduce to a surface discretization as in the previous case of PEC posts (see (3.63)).

A single PMC central post was considered with the configuration of figure $4.2, w=10$ $\mathrm{mm}$. The S-parameter results versus a FE code, ANSYS ${ }^{\circledR}$, are shown in figure 4.7 and 4.8. Only 10 even modes were required to obtain these results. The ANSYS ${ }^{\circledR}$ data was obtained by cutting a cylindrical hole with dimensions as the PMC post to be considered into a section of rectangular waveguide. Natural boundary conditions on the cylindrical surface correspond to a PMC post configuration. The standard Dirichlet boundary conditions were applied on the waveguide side and top boundaries.

\subsection{Dielectric and Magnetic Posts}

The previous examples uses both terms of the surface integral in (2.8), in which the electric and magnetic dyadic Green functions are required. The finite element section was not required for these cases. A selection of dielectric and magnetic post test cases is presented to demonstrate the ability of the Huygens principle implementation to predict accurate results compared to another numerical code.

\subsubsection{Test example 1: A thin Magnetic Post}

An off-centre thin post with magnetic and dielectric properties is selected with the configuration shown in figure 4.3. The parameters are $\epsilon_{r}=5, \mu_{r}=20, w=2 \mathrm{~mm}$ and $t=3.43 \mathrm{~mm}$. Thin posts are in general not as demanding as thicker ones, although the off-centre position does require more modes than a central post would. The number of $T E_{p 0}$ modes (both even and odd due to lack of symmetry) is 40 . The $\mathrm{S}$ parameter results are shown in figures 4.9 and 4.10 . 


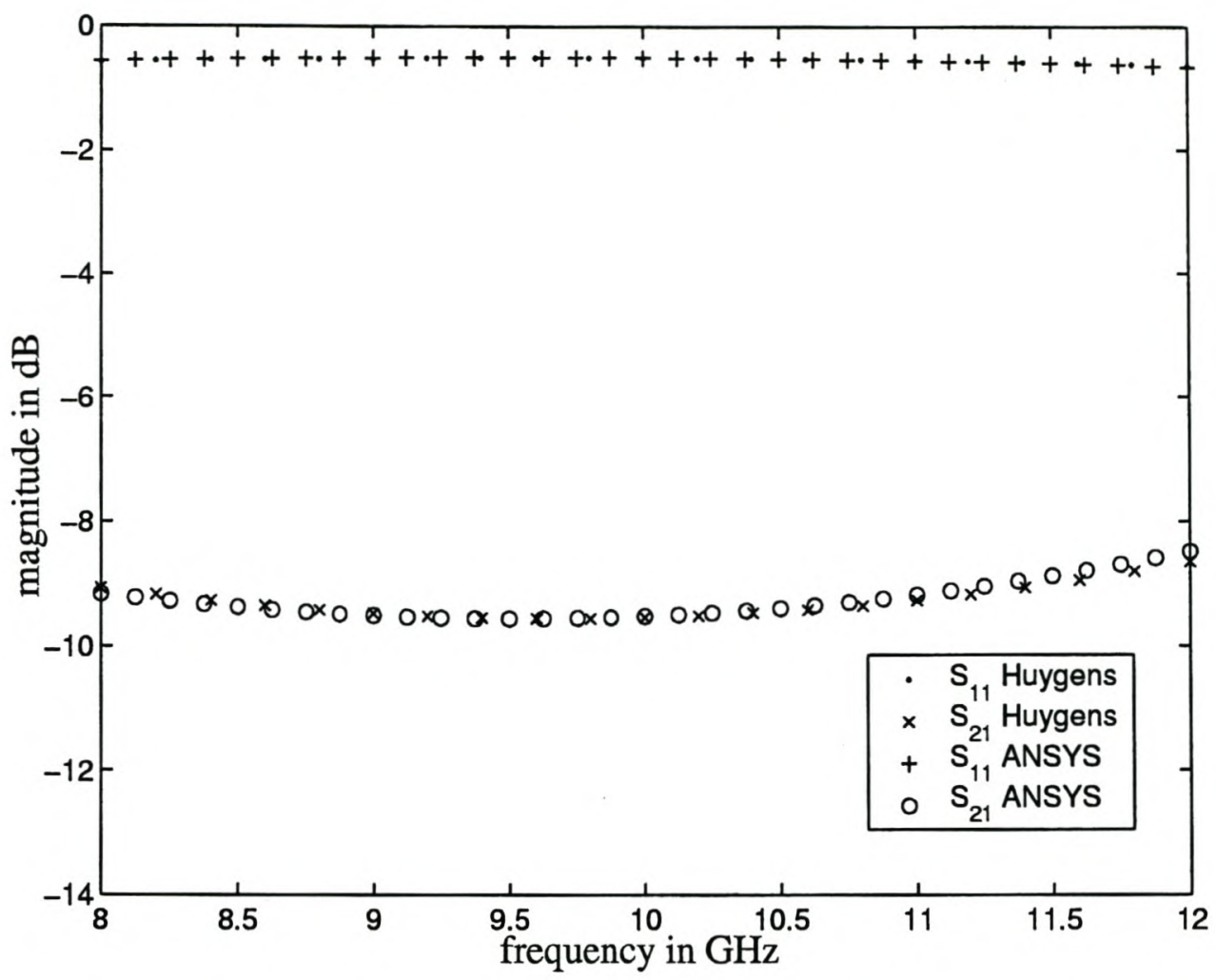

Figure 4.7: $S_{11}$ and $S_{21}$ magnitude for a PMC post

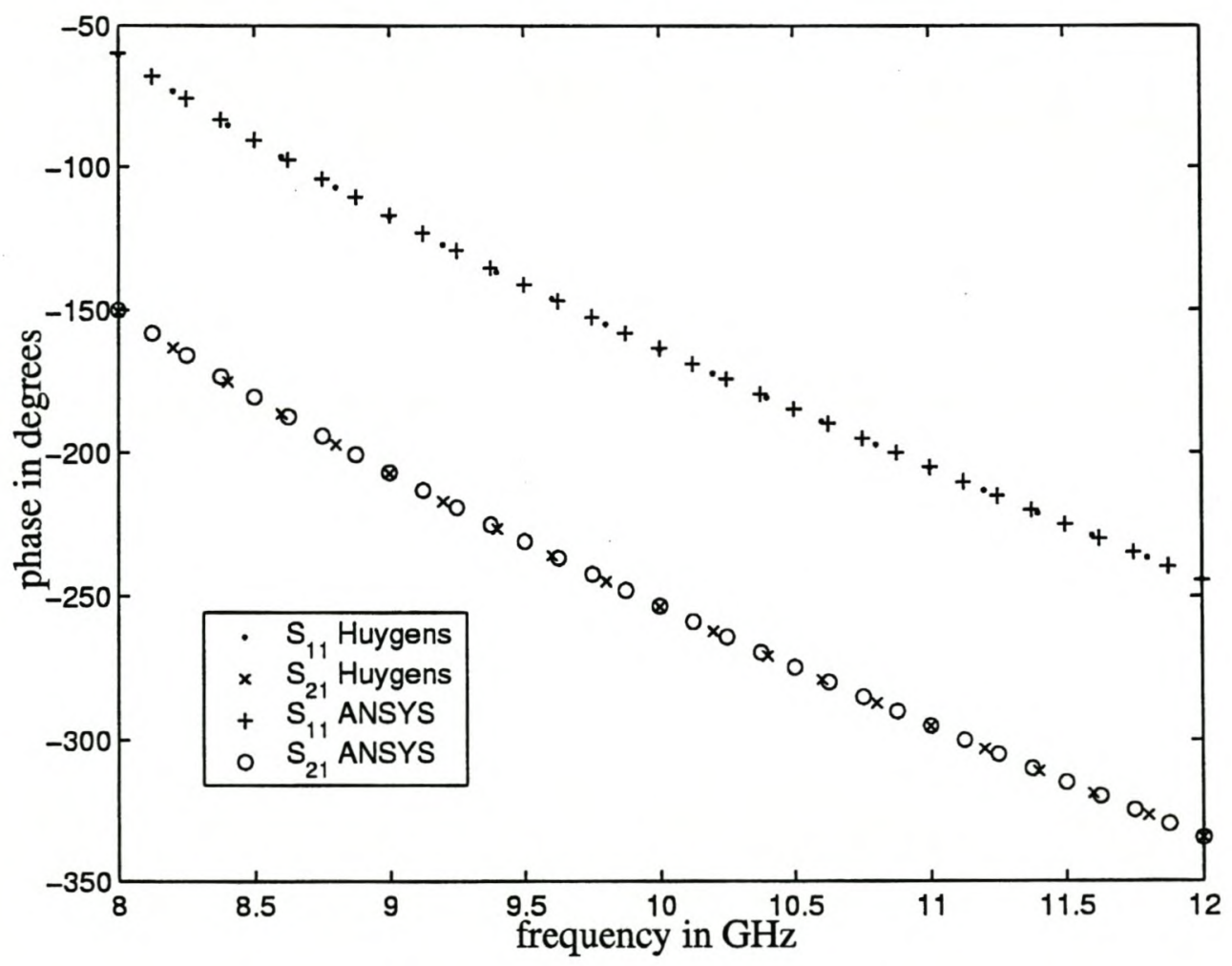

Figure 4.8: $S_{11}$ and $S_{21}$ phase for a PMC post 


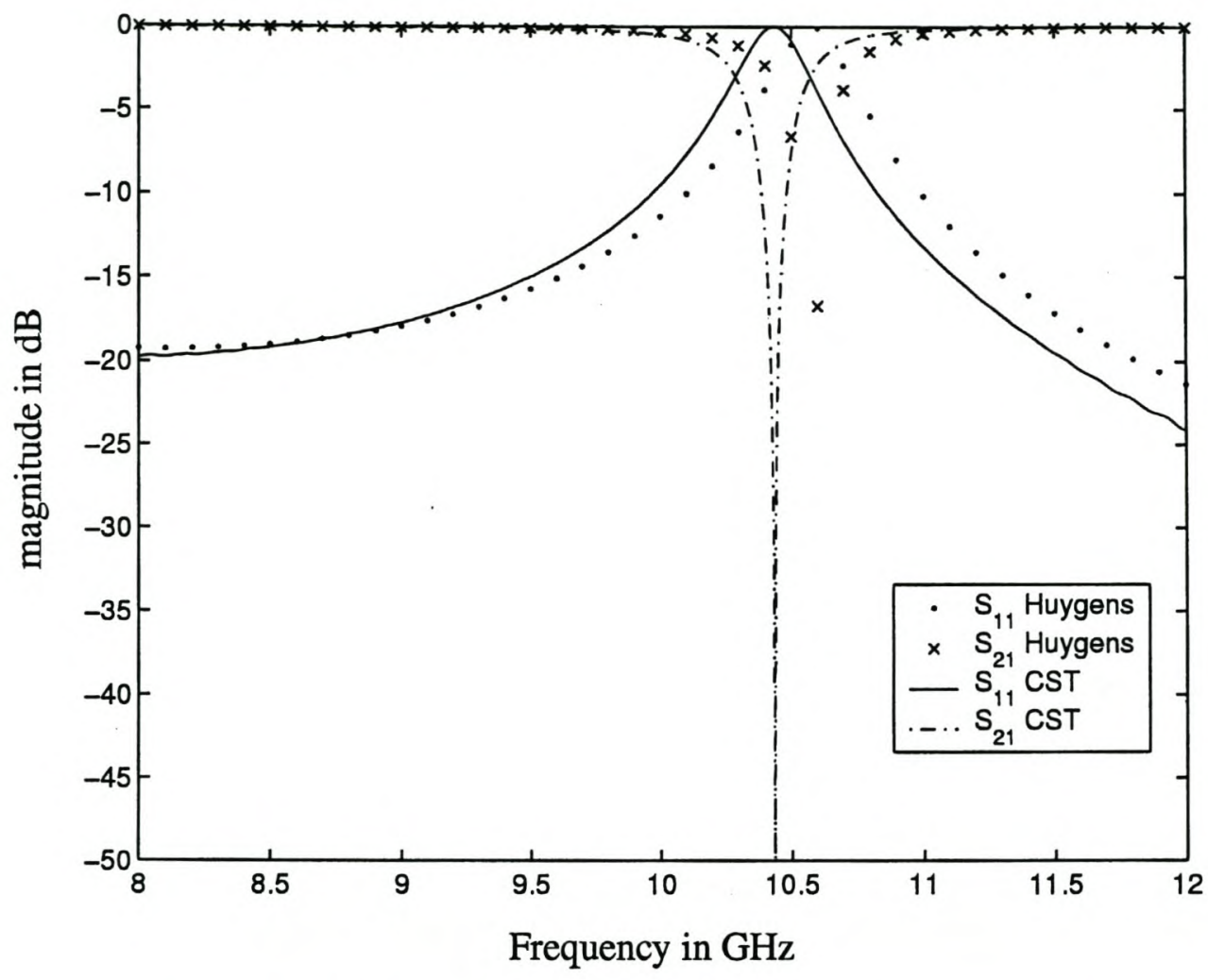

Figure 4.9: $S_{11}$ and $S_{21}$ magnitude for a thin magnetic post

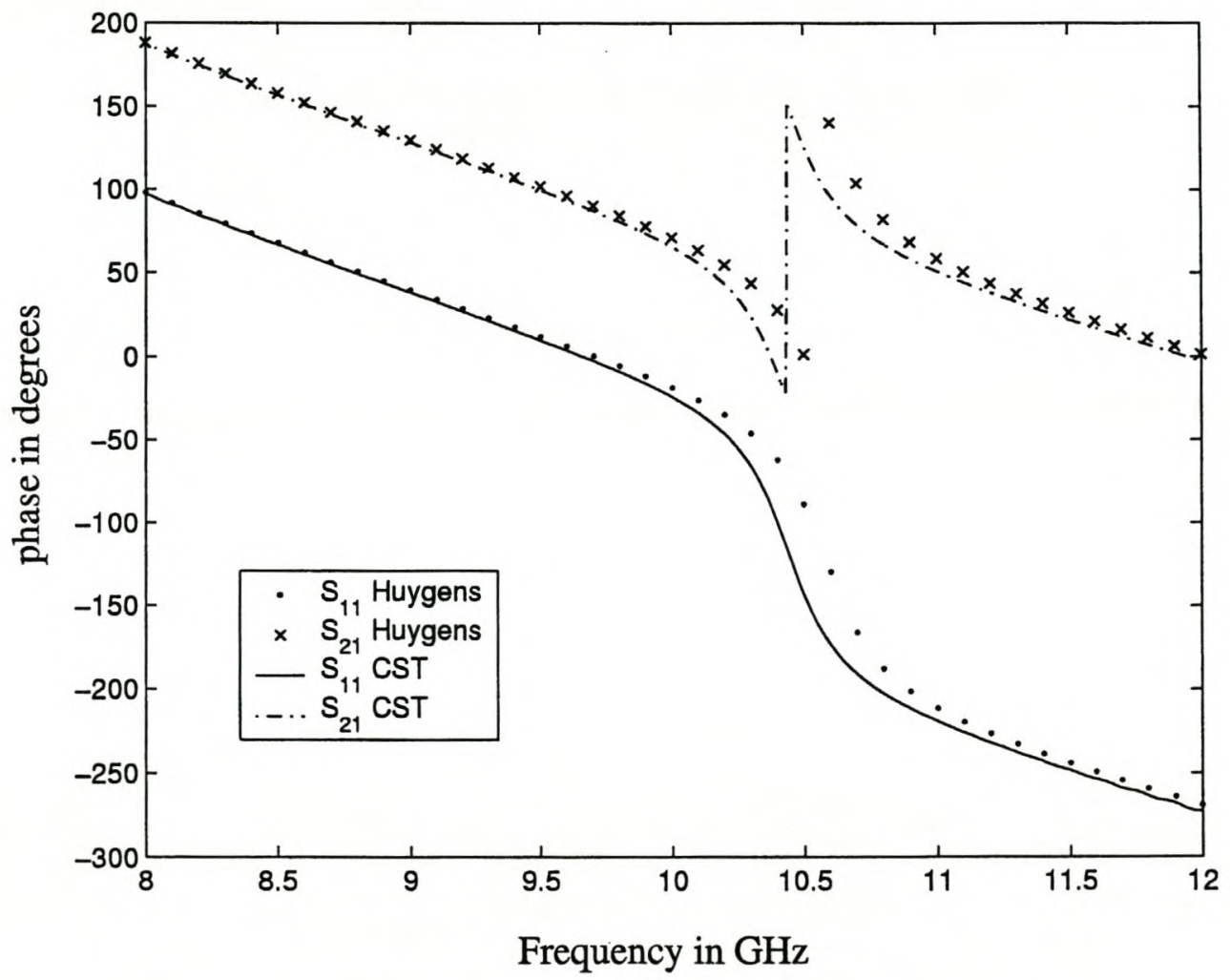

Figure 4.10: $S_{11}$ and $S_{21}$ phase for a thin magnetic post 


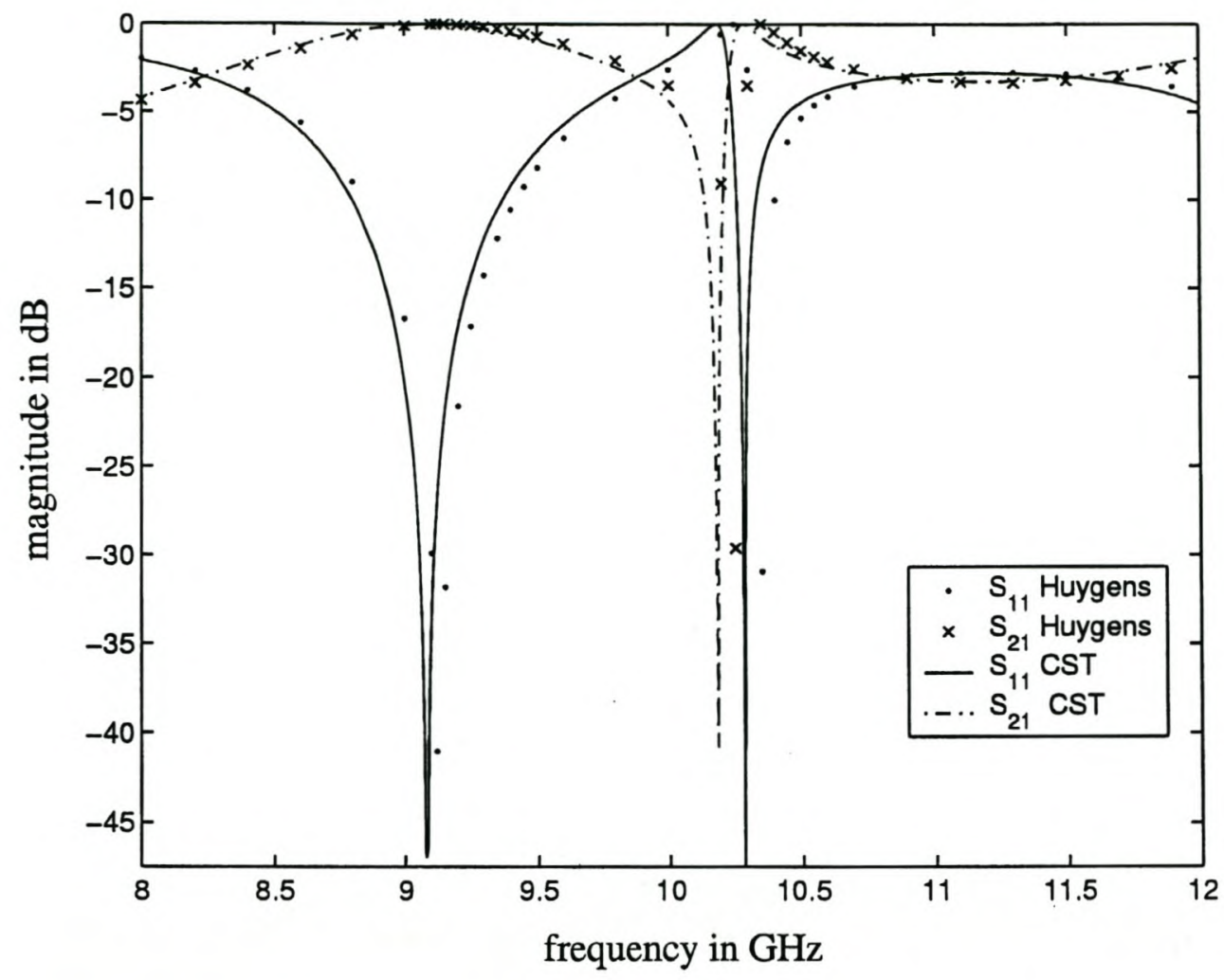

Figure 4.11: $S_{11}$ and $S_{21}$ magnitude for a thick dielectric post

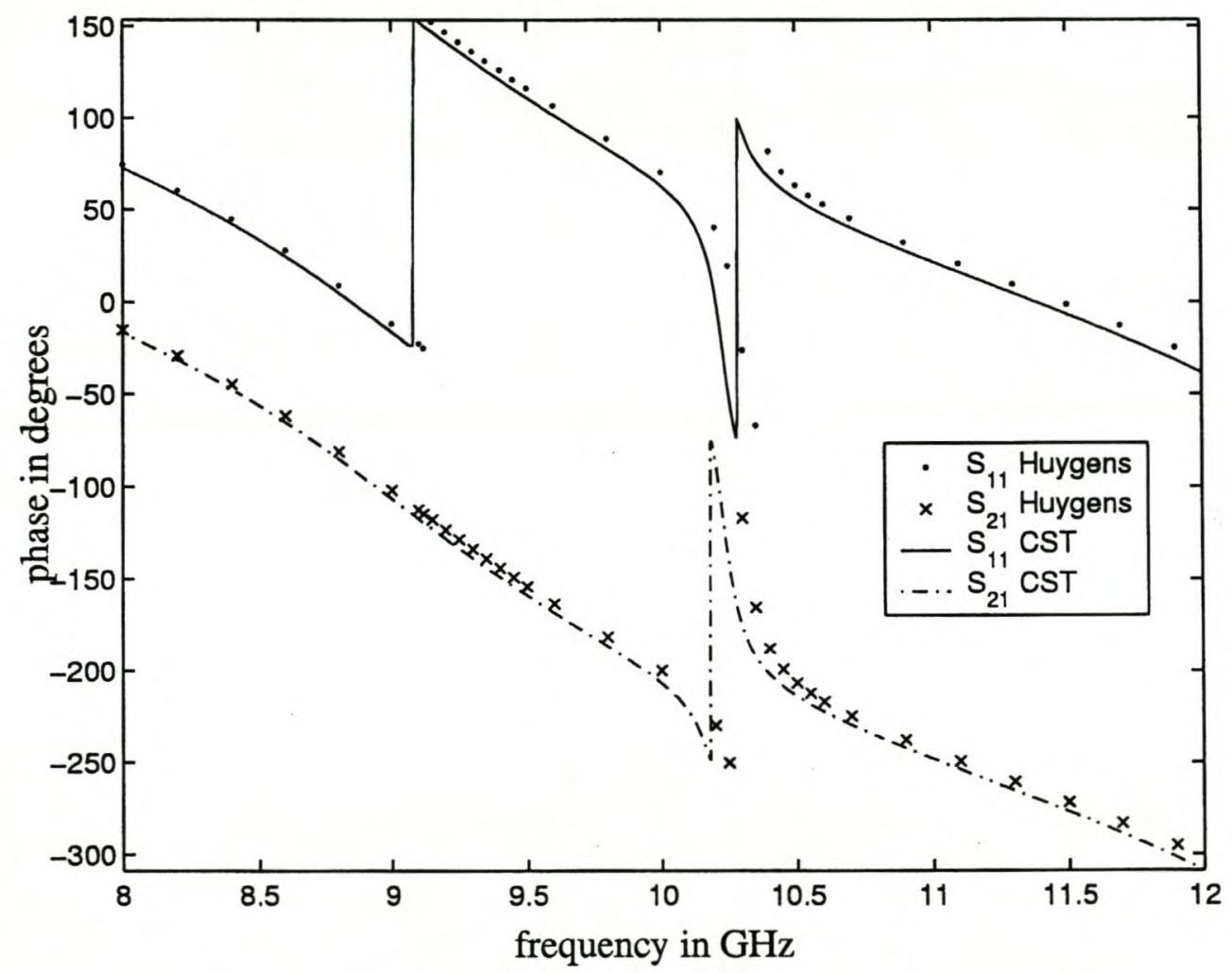

Figure 4.12: $S_{11}$ and $S_{21}$ phase for a thick dielectric post 


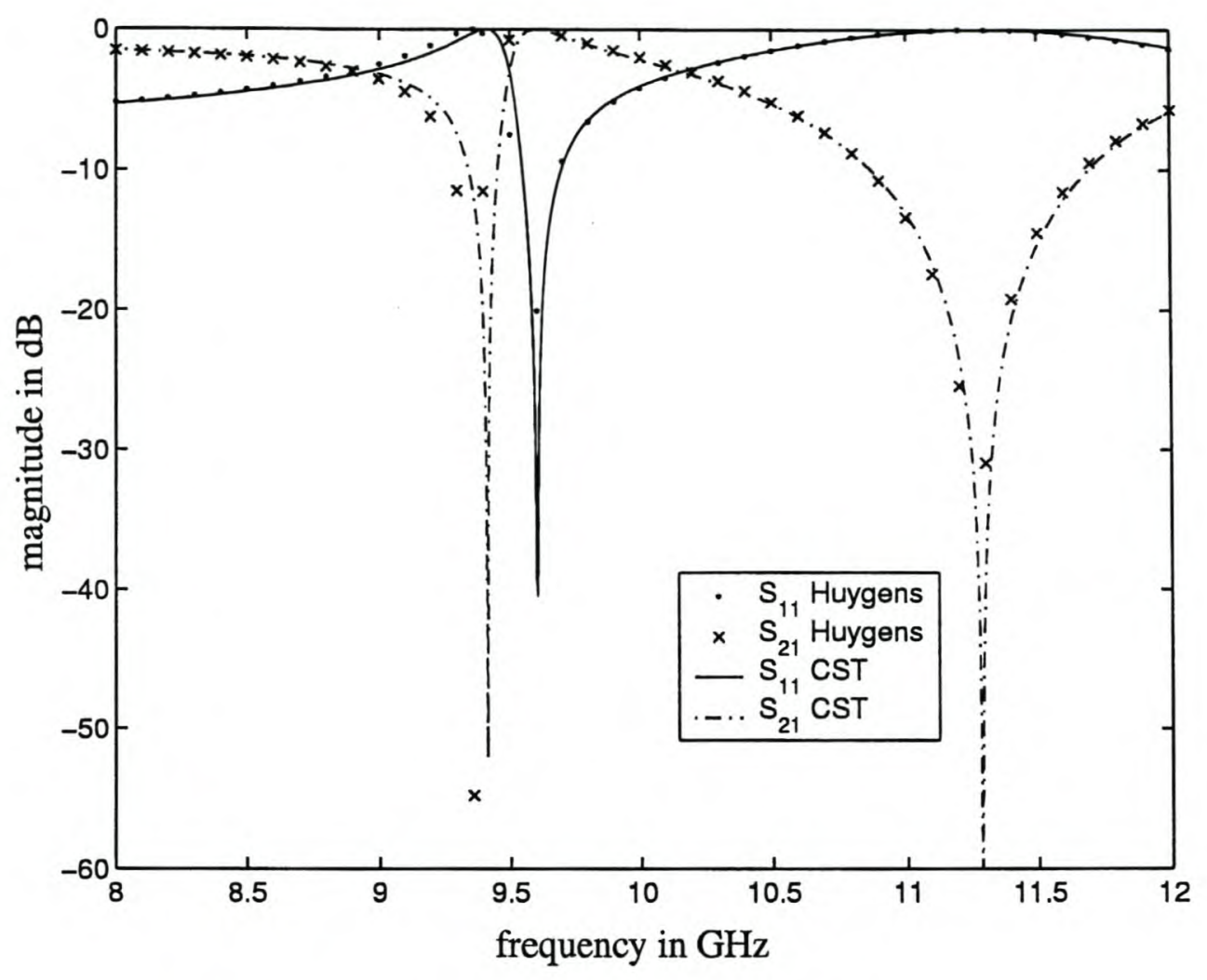

Figure 4.13: $S_{11}$ and $S_{21}$ magnitude for two posts

\subsubsection{Test example 2: A thick Dielectric Post}

This test is a much larger obstacle. A thick dielectric centre post is considered with geometry as in figure 4.2. The parameters are $\epsilon_{r}=5.67, \mu_{r}=1$ and $w=16 \mathrm{~mm}$. The results shown in figures 4.11 and 4.12 were produced by summing 20 even $T E_{p 0}$ modes. Once again a frequency shift of less than $1 \%$ is observed at resonance.

\subsubsection{Test example 3: A multiple Post Configuration}

A good demonstration of the ease with which the formulation handles separate objects is more than one post spaced some distance apart. Two dielectric posts in the configuration shown in figure 4.1 are considered. Although the discretization requirements are not high, a considerable number of modes is required to obtain the correct resonant frequency response. The parameters are $\epsilon_{r}=38.5, \mu_{r}=1, w=1.4 \mathrm{~mm}, t_{1}=9 \mathrm{~mm}$ and $t_{2}=7 \mathrm{~mm}$. The results shown in figures 4.13 and 4.14 are produced by summing . $50 T E_{p 0}$ modes. 


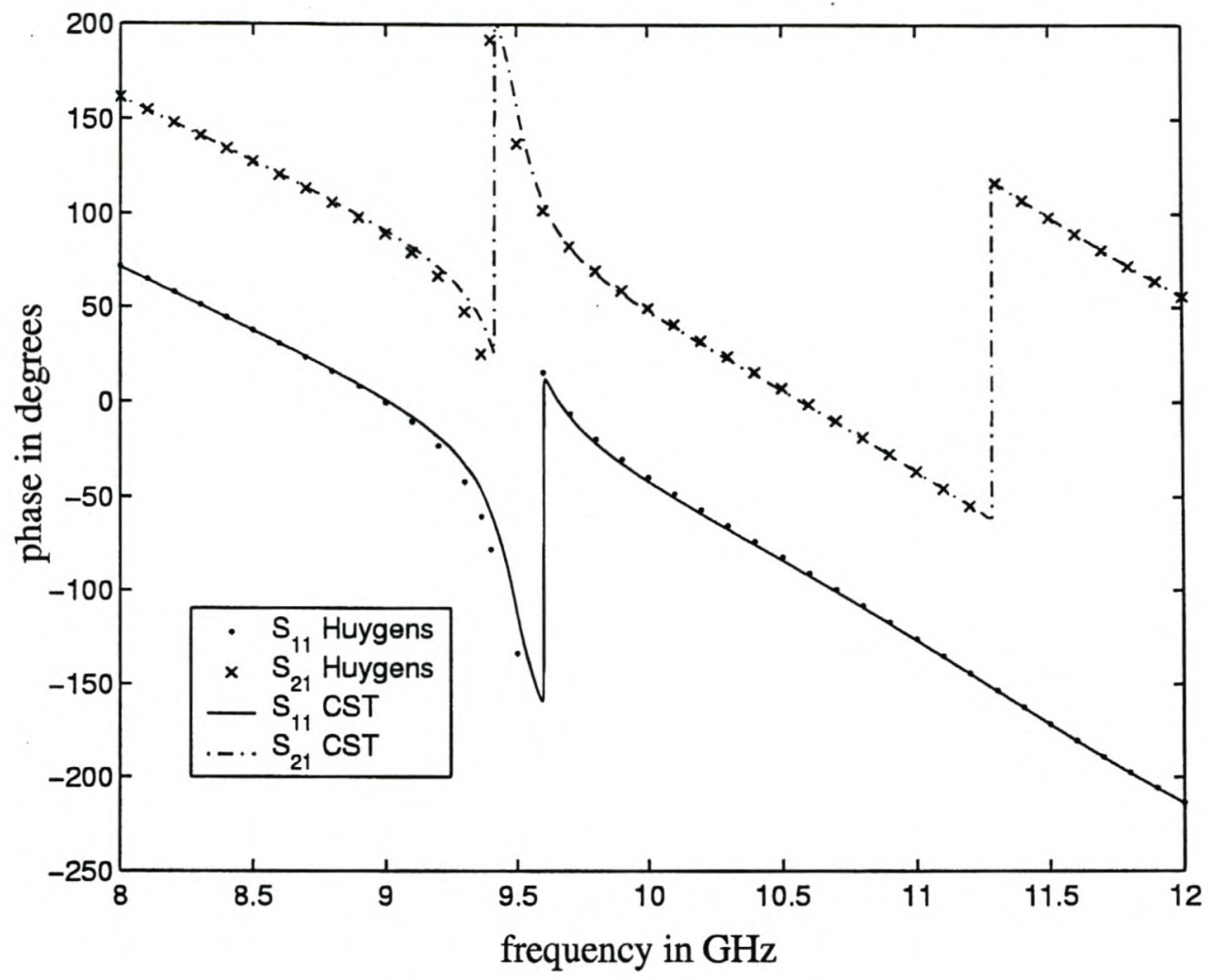

Figure 4.14: $S_{11}$ and $S_{21}$ phase for two posts

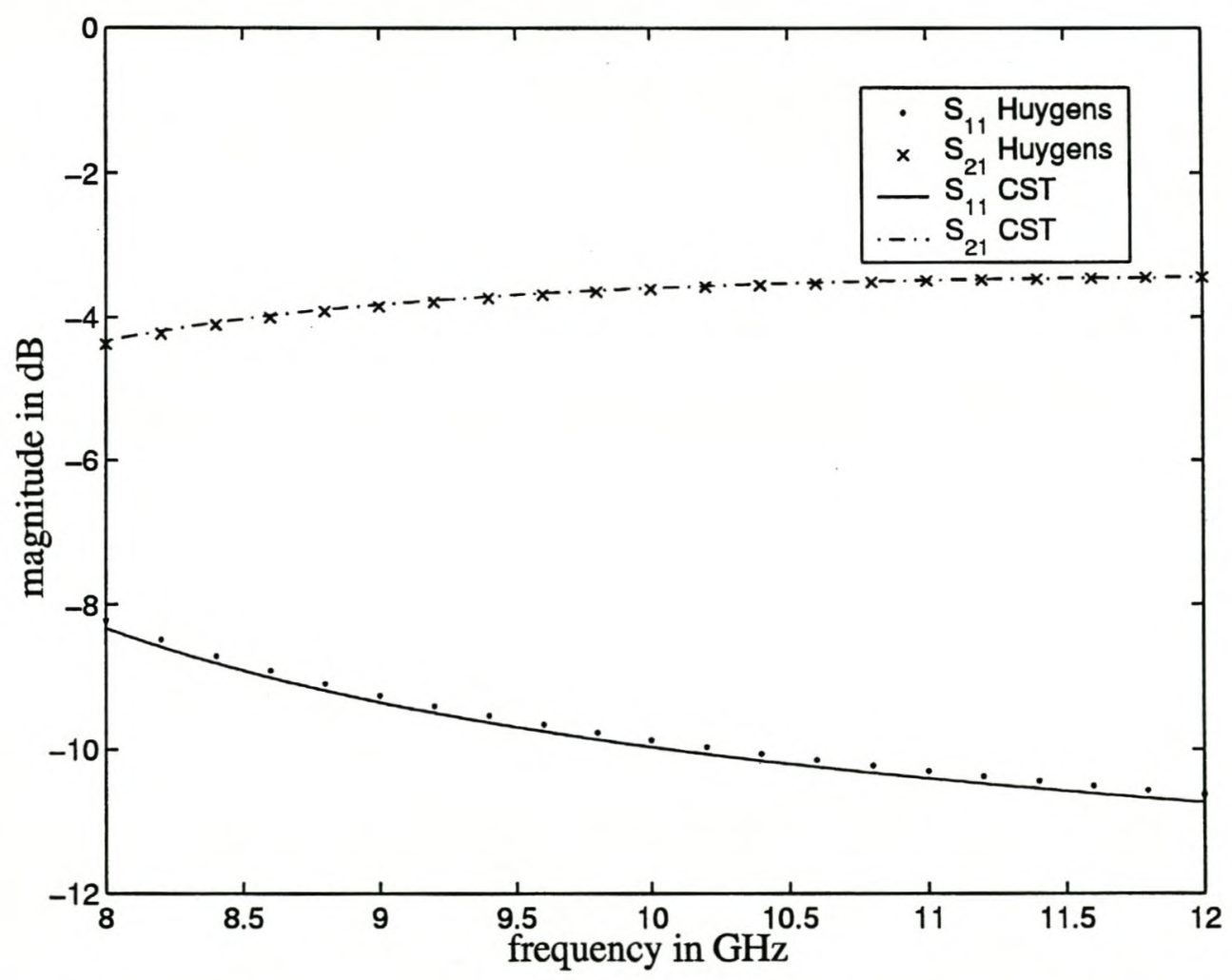

Figure 4.15: $S_{11}$ and $S_{21}$ magnitude for a lossy post 


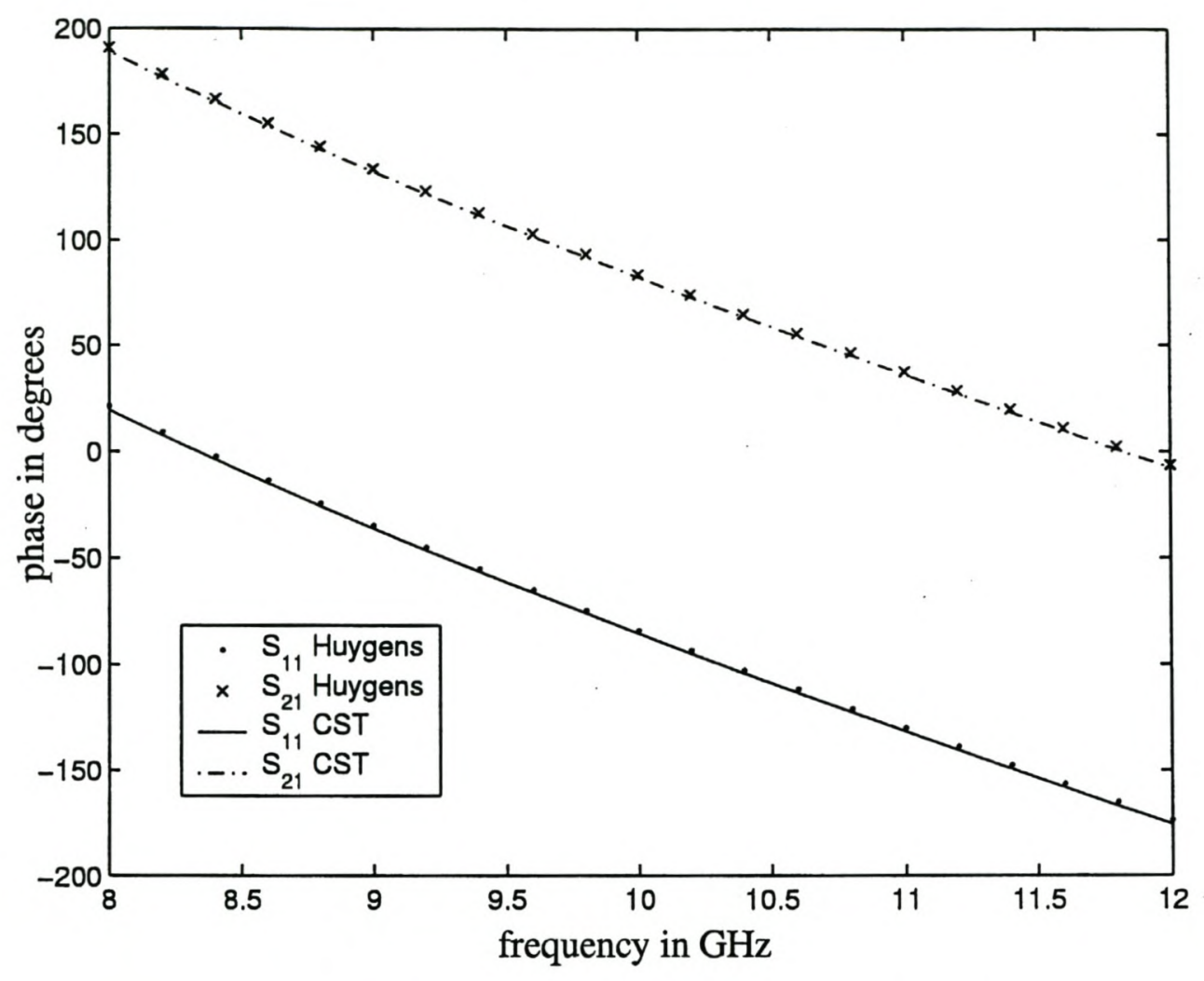

Figure 4.16: $S_{11}$ and $S_{21}$ phase for a lossy post

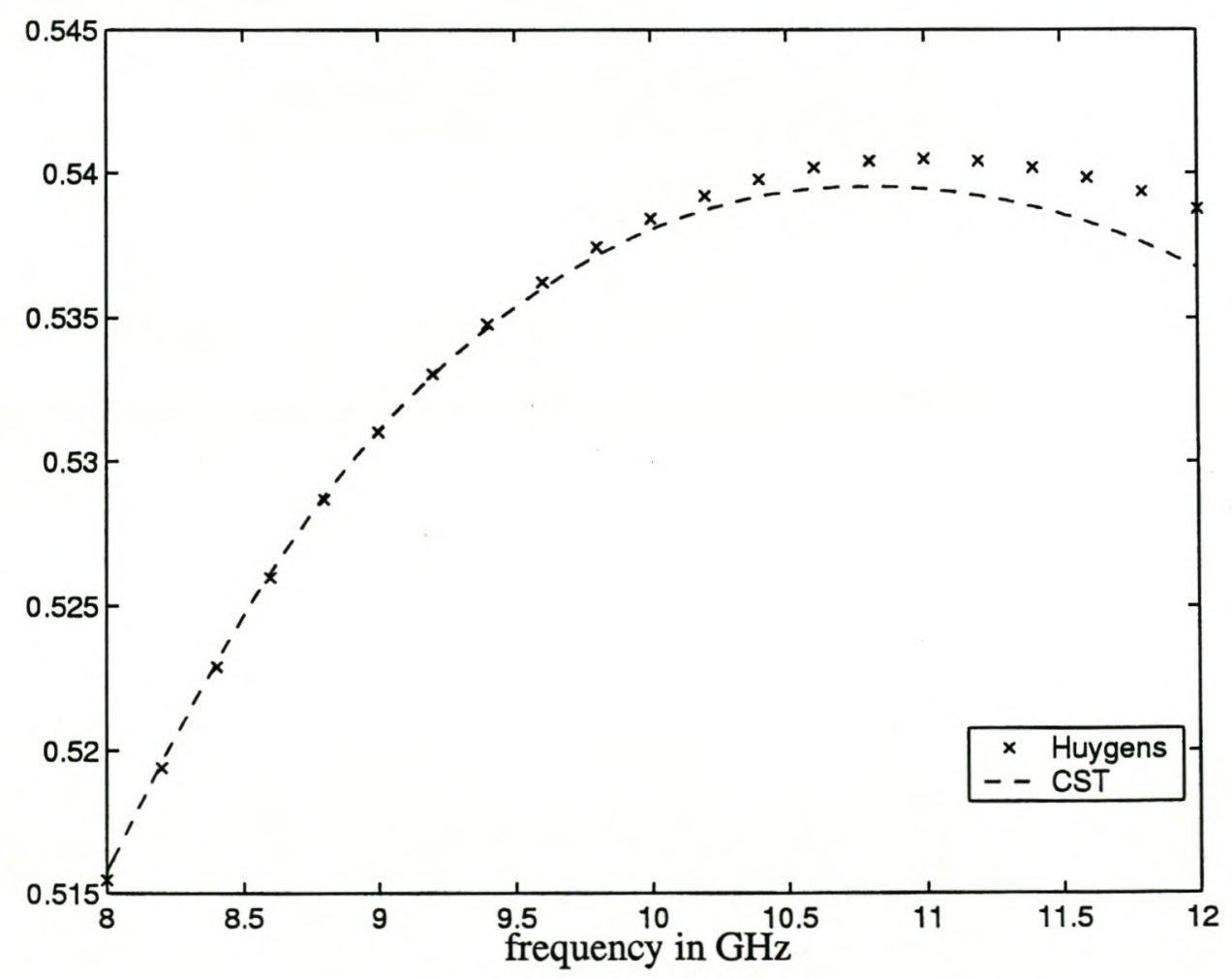

Figure 4.17: $S_{11}$ and $S_{21}$ power sum for a lossy post 


\subsubsection{Test example 4: A lossy Dielectric Post}

A reasonably sized central post with losses is considered. The central position reduces the modal requirement by half. This test case demonstrates that results for lossy posts are very accurate. The ability of the Huygens technique to handle lossy materials is inherited from the Finite Element method, and requires no modification of the surface integral expressions.

A lossy, central post with parameters $w=4 \mathrm{~mm}, \epsilon_{r}=2$ and $\tan \delta=2$ at $8 \mathrm{GHz}$ is considered. The $\mathrm{S}$ parameter results are shown in figures 4.15 to 4.17 . Only 10 even $T E_{p 0}$ modes are required. Note the slight deterioration visible in figure 4.17 at the higher frequencies, due to a constant mesh size used for the entire frequency band.s

\subsection{Convergence Requirements}

Two simple test examples are chosen to examine the convergence behaviour of a Huygens solution. The geometry is as shown in figure 4.2, with $\epsilon_{r}=5.67$ and $w=\frac{a}{2}$ or $w=\frac{a}{5}$ respectively. The discretization is as regular as possible, i.e. the volumes of elements are all very similar. The number of integration points are chosen to be reasonably high: 8 points along the width of a surface patch element. Note that the integration along the $y$-axis of each element is performed analytically. A 2D representation of the relative error against number of modes and the number of volume elements is shown in figures 4.18 and 4.20. The convergence against the number of elements is steady as expected for a standard FE solution.

When considering the number of modes, the question is, how many modes should be included? Does the solution deteriorate when an excessive number of modes are included? In figures 4.19 and 4.21, the relative $S_{11}$ error is plotted against number of modes for the same discretizations used in figures 4.18 and 4.20 . One can see that the error decreases up to a point, after which the error fluctuates erratically. This is mainly due to the numerical integration scheme, which limits the number of modes that can be represented. In both cases, the roughest discretization of only 8 elements produces inaccurate results since the geometry of the post cannot be accurately represented. As the mesh size increases, corresponding to an increase in degrees of freedom, the error drops in both cases. Clearly the smaller central post requires less waveguide modes and less elements to obtain a corresponding error in the results than the larger post. Since the discretization of the smaller post is much finer, the point at which the $\mathrm{S}$ parameter results deteriorate is not as clear as in the larger post case. 


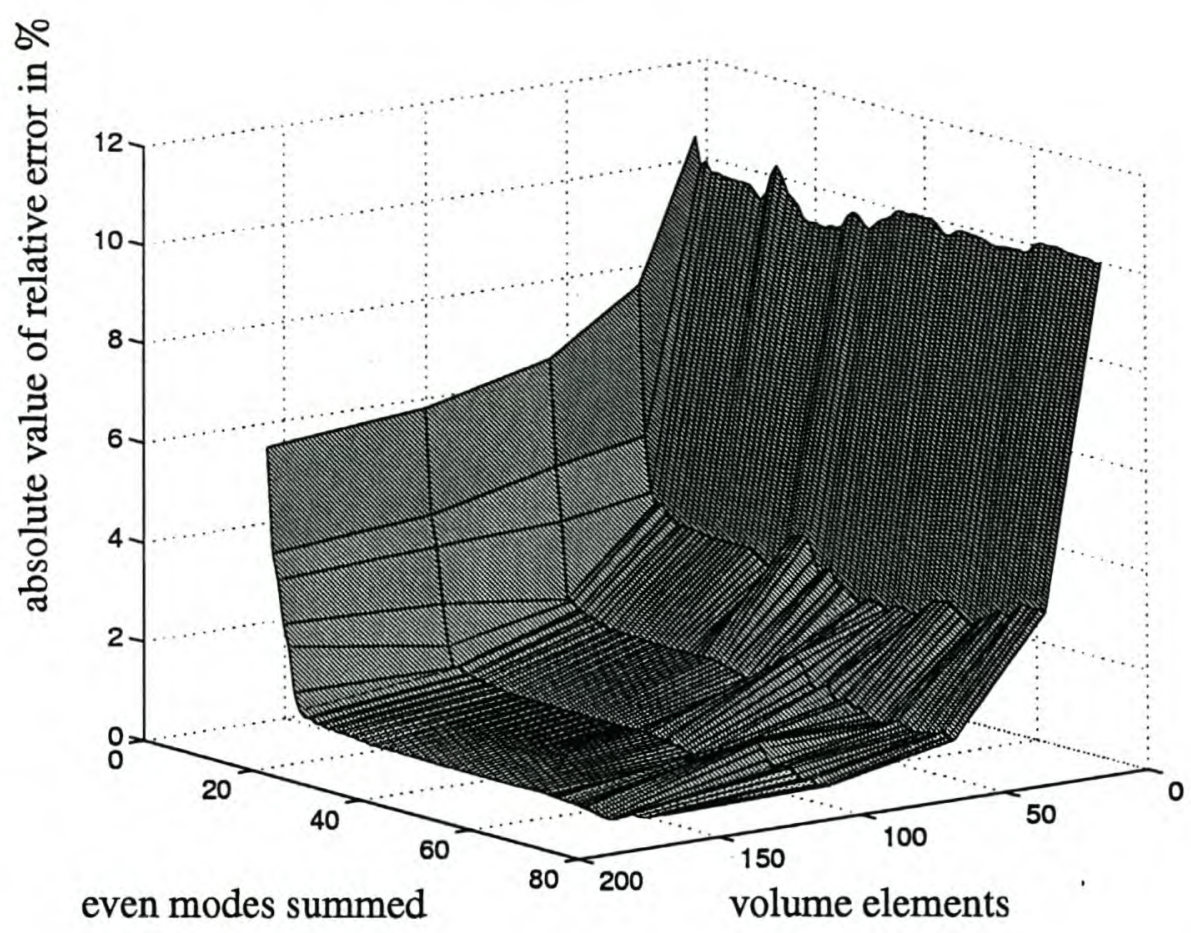

Figure 4.18: $S_{11}$ error relative to CST Microwave Studio ${ }^{\circledR}$ results for an $\frac{a}{2}$ width centre post. The variables are the number of even waveguide TE modes considered and the number of volume elements. 8 integration points per element were used.

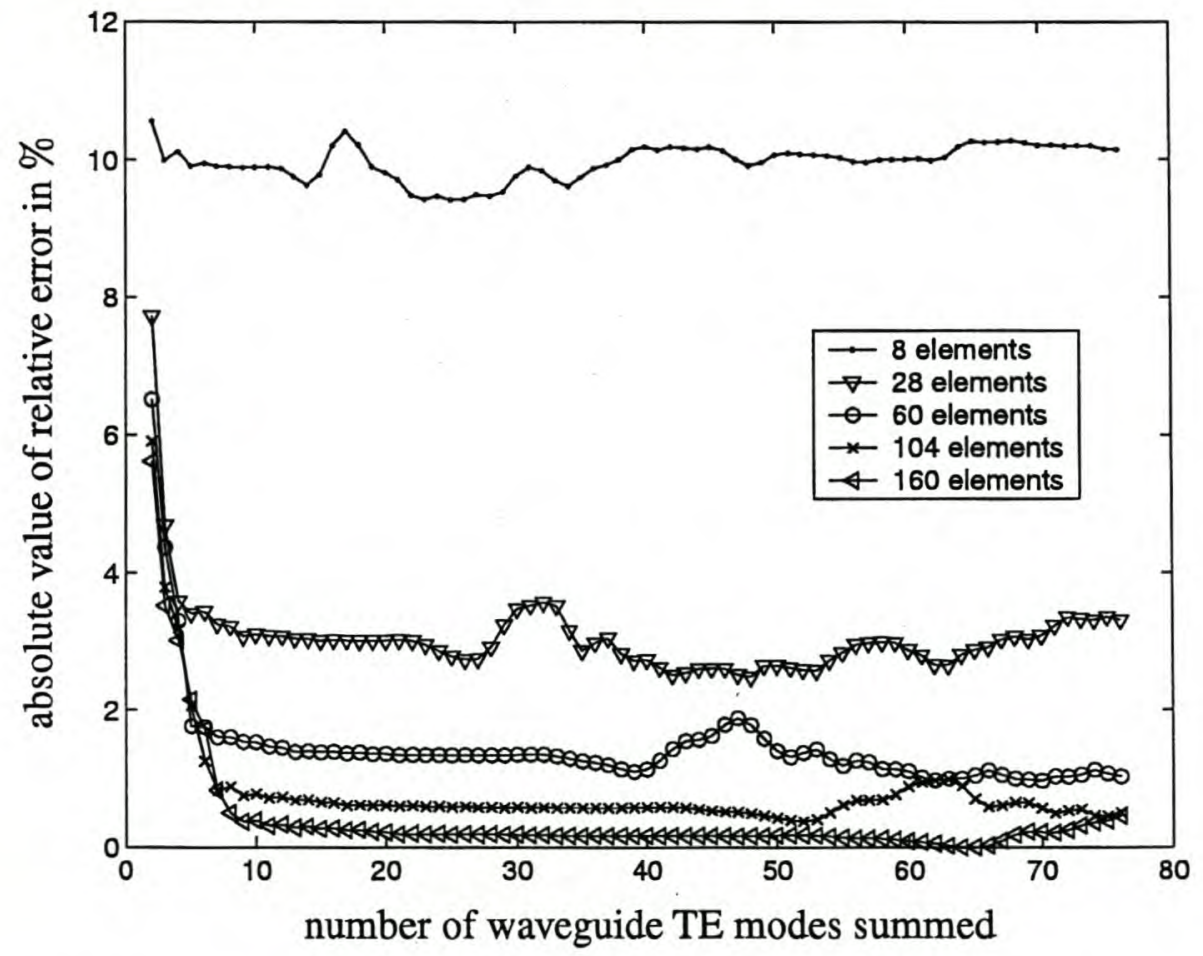

Figure 4.19: $S_{11}$ error relative to CST Microwave Studio ${ }^{\circledR}$ results against number of even waveguide modes. 


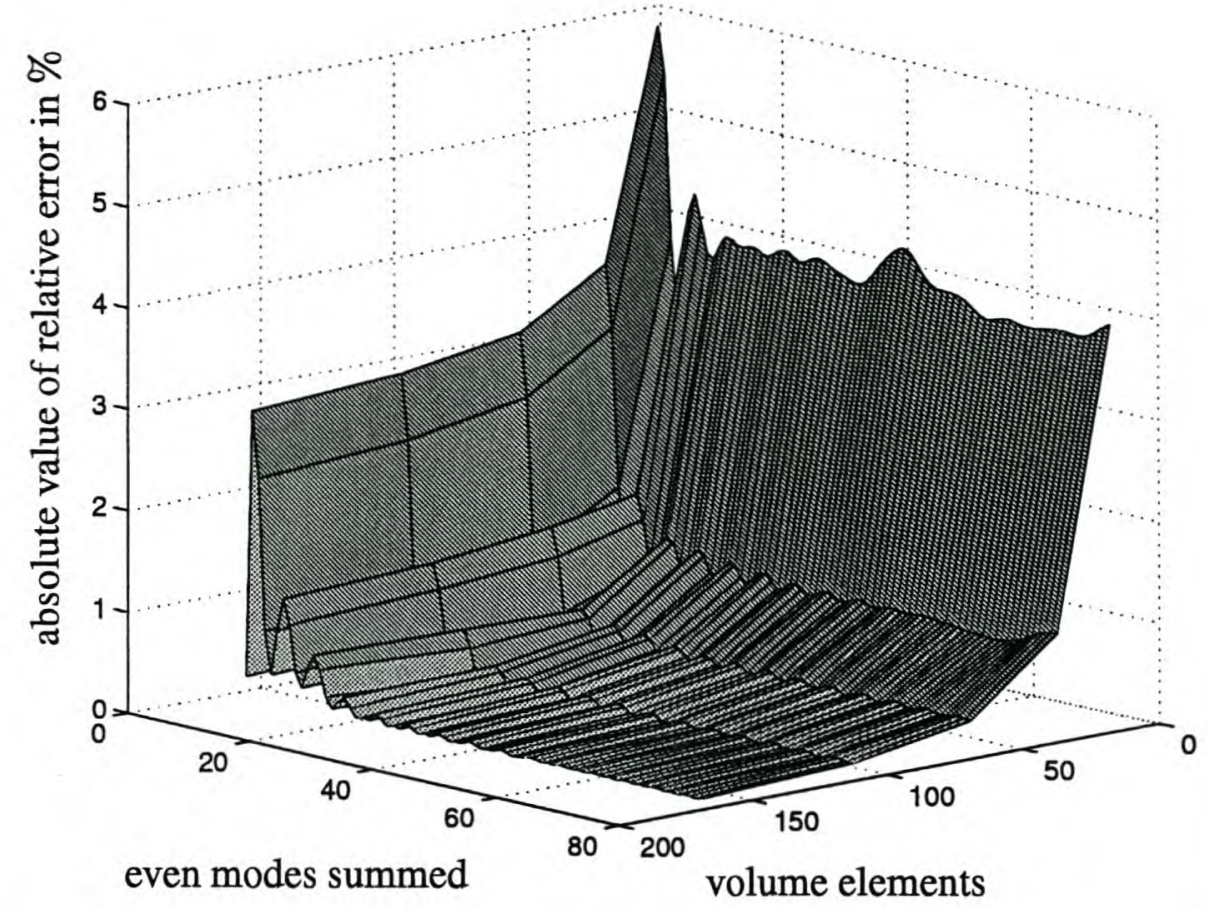

Figure 4.20: $S_{11}$ error relative to CST Microwave Studio ${ }^{\circledR}$ results for an $\frac{a}{5}$ width centre post. The variables are the number of even waveguide TE modes considered and the number of volume elements. 8 integration points per element were used.

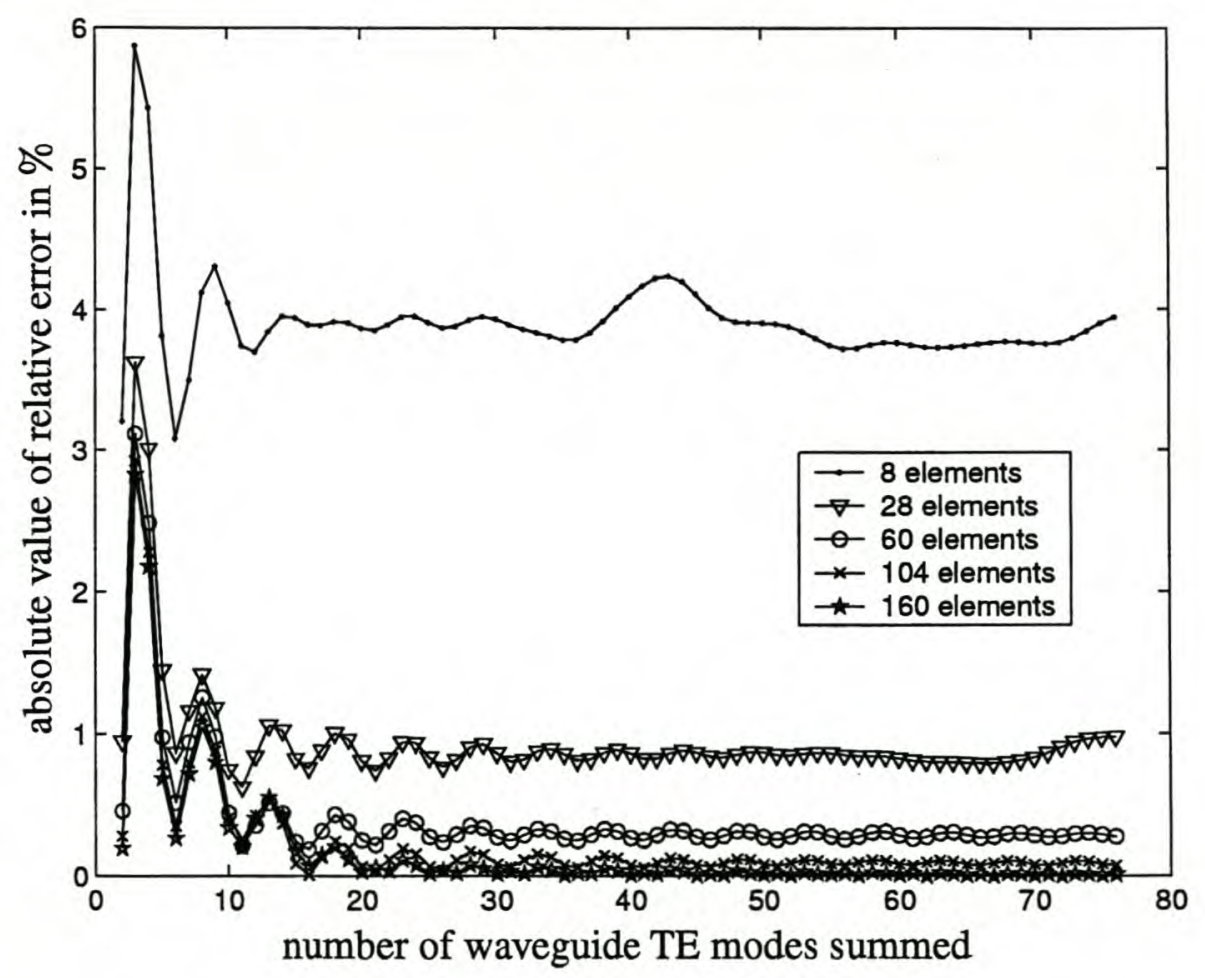

Figure 4.21: $S_{11}$ error relative to CST Microwave Studio ${ }^{\circledR}$ results against number of even waveguide modes. 
To choose the correct number of modes for a given problem, the nature of the obstacle must be considered. Small central obstacles with low permittivities can often be modelled accurately with only a few modes. The position of the obstacle in the waveguide also has a large influence. When an obstacle requiring many modes is considered, the mesh has to be refined correspondingly to be able to accurately represent the field variation produced by these modes.

An indication of suitability of the mesh size and mode number relationship can be obtained by looking at $\left|S_{11}\right|^{2}+\left|S_{21}\right|^{2}$. For lossless cases this must be 1 . Based on numerical experiments, an error of more than 1 percent here gives an indication that the mesh elements are too large and that a mesh refinement or a higher order basis set implementation is required. The result of such an error is that both S-parameters will not be equally accurate. For thick posts, or posts with high relative permittivity, an extension to a higher order basis set implementation could be used to avoid very fine mesh discretizations.

\subsection{Summary}

S-parameter results for various size posts were obtained and found to be in good agreement with a commercial time domain code. These test cases show that the Huygens formulation is capable of providing accurate results. The number of modes required in many cases required are not very large; in fact it is confirmed that the single infinite summation of modes required theoretically may be replaced by a much smaller number of modes in practice. It depends very much on the type of obstacle inserted into the waveguide. This fact, combined with the volume mesh contained only inside the scatterers, makes the Huygens formulation a very suitable choice for scattering problems involving dielectric materials in waveguide. It was demonstrated that the strength of the Huygens technique lies in problems with multiple smaller obstacles rather than very large objects filling a substantial portion of the waveguide cross-section. The latter case may be equally well considered with a full Finite Element discretization and solution.

Considering PEC scatterers alone, these simplify to a moment method type implementation and do not require the full Huygens formulation, in fact the ability to solve for metallic scatterers in waveguide is well established. However, the Huygens' principle is not limited to scatterers containing either metallic or dielectric parts, but may be implemented to allow a combination of both, although no such results are shown here.

On the topic of numerical efficiency a comparison of execution speed is not practical 
as the code was implemented in MATLAB ${ }^{\circledR}$, notoriously slow with non-vectorized operations. In addition, commercial codes typically are optimized for execution speed. The results obtained for dielectric posts are the first results available for the verification of this formulation. These results demonstrate that the technique is indeed suitable for the solution of scattering problems in waveguide. 


\section{Chapter 5}

\section{Conclusions and Recommendations}

\subsection{Introduction}

The benefit of the Huygens formulation is most evident when a number of relatively small obstacles are introduced in an otherwise empty waveguide. The spacing of these obstacles relative to one another has no impact on the computational burden. This is in contrast with other techniques that discretize the entire volume in the vicinity of the scatterers, usually including surrounding empty waveguide.

In a comparison with a waveguide finite element solution of a waveguide with obstacle, where discretization is made with only the dominant mode considered, the Huygens technique requires a surface discretization for a number of higher order modes. The requirements for the number of modes to be considered for a good solution are not excessive, however the surface mesh must be suitably fine to support these modes and by implication this affects the volume finite element mesh that must link to the surface patch mesh. The result is that the meshing of the scatterer itself is finer than it will be for a dominant mode finite element analysis. For this reason the benefits of the technique decrease as the volume of the scatterer increases relative to the surrounding empty volume up to the point where a finite element analysis would be more effective.

\subsection{Evaluation of the Technique}

From a practical point of view one expects numerical codes to provide numerical data that correspond to physical measured data. For the purposes of this thesis the focus is on the comparison of numerical data with other existing codes, assuming that these codes provide data that corresponds to physical reality. 
For small obstacles the results obtained compared to a time domain code and a FE code are suitably accurate. As the diameter of the posts increased, the mesh requirements increase correspondingly.

A combination of loads spaced some distance apart may now be analysed without segmentation of the waveguide as would typically be required [48]. Especially if the loads are quite small the method is much more efficient than the other techniques discussed in chapter 1 .

A test example with two dielectric posts demonstrates the ability of the method to provide accurate results around resonance.

Some problems may be better solved by other techniques. These are problems involving only metallic discontinuities, such as metallic posts and waveguide junctions. Also the analysis of very large obstacles will not benefit substantially from this technique, but are more suited to a standard finite element analysis. Dielectric obstacles, or combinations of dielectric, magnetic and metallic obstacles are very suitable problems for this technique.

An important observation based on the results presented in chapter 6, is that often, surprisingly few waveguide modes are required to obtain good S-parameter data. This corresponds with the approach in [2] where only a few modes were included to provide remarkably accurate results. However, numerically the physical geometry must be approximated by a simplified geometry allowed by the prismatic, tetrahedral or rectangular basis elements. In some cases the accuracy is limited by the geometric approximation of the object.

\subsection{Applications}

Some of the examples in the previous chapter are typical configurations for waveguide filters. Usually the filter elements are spaced some distance apart, which would require a much larger number of degrees of freedom to model in a standard finite element approach.

Commercial microwave heating applications, for example the drying of wood, require the modelling of microwave applicators with loads that are lossy. Without the modelling of the dielectric losses the analysis of power distribution in a load cannot be made. The power density in the load is used to establish the heating effect of the microwave oven. The applicators have various shapes but may be in the form of a tunnel 
oven. The load itself is usually irregularly shaped and does not occupy the entire crosssection of the applicator for practical reasons. A method of moments implementation appears in [49] where it is claimed to be much more effective than a FDTD approach. In [50] a mode-matching and FE technique is applied to cavities with inhomogeneous materials. These type of problems are very suitable candidates for modelling with the use of the Huygens formulation, substituting the appropriate Green functions in each case, which could provide very effective solutions to the field and power distributions in the loads. An example in chapter 4 clearly demonstrates the accuracy with which lossy obstacles can be modelled.

\subsection{Possible Extensions of the Application}

The test cases chosen have demonstrated the ability of the Huygens technique to solve waveguide scattering problems. A number of aspects may be included as extensions to the implementation in future: more general geometric modelling and more efficient matrix equation solutions for much larger problems. An upgrade to the higher order basis set proposed will also increase the accuracy of results obtained or would provide the same accuracy with less elements.

\subsubsection{Geometric Extensions}

The most pressing extension required is the ability to model arbitrary 3D obstacles with any restriction on geometric shape removed. The geometric modelling implemented at present allows only objects without variation in the y-direction to be modelled. These posts had to extend from the bottom to the top waveguide walls. The prismatic elements chosen are very suitable for this purpose. In the case of more general obstacles, tetrahedral finite elements would be a more suitable choice, with various higher order basis sets already in use. If objects with rounded shapes are to be considered, another option is the three dimensional covariant projection elements in [51]. These elements model curved geometries very effectively. The waveguide cross-section is not limited to rectangular shapes, any other practical shape could be analysed with this technique.

With the extension to more general geometries the full set of TE and TM modes will be required in the analysis. All 9 components of each dyadic Green function would be required. The main effect of this will be to increase execution times. 


\subsubsection{Efficient Matrix Solutions}

In the past few years numerous papers on the use of wavelets in numerical electromagnetics have been published $[52,53,54,55,56]$. Typical method of moments matrices are dense, unlike finite element matrices, which are banded and sparse. Difficulties arise when computing the solution to matrix equations $[A]\{x\}=\{b\}$, resulting from a method of moments implementation, in terms of the number of operations required. This requires $O\left(Q^{3}\right)$ operations, with $Q$ the dimension of $[A]$. In contrast a matrix equation solution after a wavelet transform requires $O\left(Q^{2}\right)$ operations [57].

Two different approaches using wavelet theory are common. The first transforms a full dense matrix, found after a method of moments application, into a sparse matrix via a wavelet transformation followed by a threshold operation that discards small entries $[54,56]$. The transformed matrix equation is then solved and the original solution obtained. The second approach uses wavelets as bases in the moment method application $[52,53,55]$. An important issue is the matrix condition number after transformation, which reportedly shows a marked deterioration [56].

\subsection{Conclusion}

A novel numerical technique applicable to arbitrary scatterers inside hollow waveguides was introduced recently. In this thesis, the first implementation of this method was presented. The scatterer itself is modelled with prismatic finite elements, while a quasi method of moments analysis is executed on the surface of the scatterer. The inner volume and the surface of the scatterer or load is linked through the tangential electric and magnetic fields on the scatterer surface. The geometries considered were limited to posts in waveguide, although the technique itself is general and can be applied to arbitrary scatterers or loads inside waveguides. S-parameter results were obtained for conducting and dielectric posts inside $\mathrm{X}$-band waveguide. These results were in good agreement with a commercial time domain code. 


\section{Bibliography}

[1] A. A. Oliner, "Historical Perspective on Microwave Field Theory," IEEE Transactions on Microwave Theory and Techniques, pp. 1022-1045, 1984.

[2] N. Marcuvitz, Waveguide Handbook. McGraw Hill, 2nd ed., 1951.

[3] N. Marcuvitz and J. Schwinger, "On the Representation of the Electric and Magnetic Fields produced by Currents and Discontinuities in Wave Guides I," Journal of Applied Physics, vol. 22, no. 6, pp. 806-819, 1951.

[4] J. Schwinger and D. S. Saxon, Discontinuities in Waveguides: Notes on Lectures by Julian Schwinger. Gordon and Breach Science Publishers, 1968.

[5] I. C. Hunter, L. Billonet, B. Jarry, and P. Guillon, "Microwave Filters- Applications and Technology," IEEE Transactions on Microwave Theory and Techniques, vol. 50, pp. 794-805, March 2002.

[6] F. Alessandri, M. Mongiardo, and R. Sorrentino, "Computer-Aided Design of Beam Forming Networks for Modern Satellite Antennas," IEEE Transactions on Microwave Theory and Techniques, vol. 40, pp. 1117-1127, June 1992.

[7] A. Wexler, "Solution of Waveguide Discontinuities by Modal Analysis," IEEE Transactions on Microwave Theory and Techniques, vol. 15, pp. 508-517, September 1967.

[8] R. F. Harrington, "Matrix Methods for Field Problems," Proc, IEEE, vol. 55, pp. 24-37, February 1967.

[9] J. J. Wang, Generalised Moment Methods in Electromagnetics. John Wiley \& Sons, Inc, p. 169, 1991.

[10] V. K. Thong, "Solutions for some Waveguide Discontinuities by the Method of Moments," IEEE Transactions on Microwave Theory and Techniques, pp. 416418, June 1972. 
[11] Y. Leviatan, D. Shau, and A. T. Adams, "Numerical Study of the Current Distribution on a Post in a Rectangular waveguide," IEEE Transactions on Microwave Theory and Techniques, vol. 32, pp. 1411-1415, October 1984.

[12] A. S. Omar and K. Schunemann, "Scattering by Dielectric Obstacles inside Guiding Structures,” MTT-S Digest, pp. 321-323, 1984.

[13] Y. Leviatan and G. S. Sheaffer, "Analysis of Inductive Dielectric Posts in Rectangular Waveguide," IEEE Transactions on Microwave Theory and Techniques, vol. 35, pp. 48-59, January 1987.

[14] C. Tai and P. Rozenfeld, "Different representations of Dyadic Green's Functions for a Rectangular Cavity," IEEE Transactions on Microwave Theory and Techniques, vol. 24, pp. 597-601, September 1976.

[15] J. J. H. Wang, "Analysis of a Three-dimensional Arbitrary-shaped Dielectric or Biological Body inside a Rectangular Waveguide," IEEE Transactions on Microwave Theory, vol. 26, pp. 457-461, July 1978.

[16] C.-I. G. Hsu and H. A. Auda, "Multiple Dielectric Posts in a Rectangular Waveguide," IEEE Transactions on Microwave Theory and Techniques, vol. 34, pp. 883-891, August 1986.

[17] M. Koshiba and M. Susuki, "Application of the Boundary Element Method to Waveguide Discontinuities," IEEE Transactions on Microwave Theory and Techniques, vol. 34, pp. 301-307, February 1986.

[18] K. Ishibashi and E. Sawado, "Three dimensional analysis of electromagnetic fields in rectangular waveguides by the boundary integral equation method," IEEE Transactions on Microwave Theory and Techniques, vol. 38, pp. 13001308, September 1990.

[19] J. P. Webb, "Edge Elements and what they can do for you," IEEE Transactions on Magnetics, vol. 29, pp. 1460-1465, March 1993.

[20] J. P. Webb and B. Forghani, "Hierarchal Scalar and Vector Tetrahedra," IEEE Transactions on Magnetics, vol. 29, pp. 1495-1498, March 1993.

[21] P. P. Silvester and R. L. Ferrari, Finite Elements for Electrical Engineers. Cambridge University Press, 3rd ed., 1996.

[22] J. L. Volakis, A. Chatterjee, and L. C. Kempel, Finite Element Method for Electromagnetics: Antennas, Microwave Circuits and Scattering Applications. IEEE Press, 1998. 
[23] D. Sun, J. Manges, X. Yuan, and Z. Cendes, "Spurious modes in Finite Element Methods," IEEE Antennas and Propagation Magazine, vol. 37, pp. 12-24, October 1995.

[24] D. Sun, J. Manges, and X. Yuan, "Spurious Modes in Finite Element Methods," IEEE Antennas and Propagation Magazine, vol. 37, pp. 12-24, October 1995.

[25] J. Jin, The Finite Element Method in Electromagnetics. John Wiley \& Sons, Inc, 1993.

[26] J. Liu, J.-M. Jin, E. K. N. Yung, and R. S. Chen, "A Fast, Higher Order Three Dimensional Finite Element Analysis of Microwave Waveguide Devices," Microwave and Optical Technology Letters, vol. 32, pp. 344-352, March 2002.

[27] J. P. Webb, "Finite Element Methods for Junctions of Microwave and Optical Waveguides," IEEE Transactions on Magnetics, vol. 26, pp. 1754-1758, September 1990 .

[28] W. B. Dou and E. K. N. Yung, "FDTD Analysis for Multiple Arbitrary Shaped Posts in a Waveguide," Microwave and Optical Technology Letters, vol. 27, pp. 1419-1430, November 2000.

[29] R. Lehmensiek and P. Meyer, "Creating Accurate Multivariate Rational Interpolation Models of Microwave Circuits by using Efficient Adaptive Sampling to Minmize the number of Computational Electromagnetics Analyses," IEEE Transactions on Microwave Theory and Techniques, vol. 49, pp. 1419-1430, August 2001.

[30] D. Arena, G. M. Ludivico, G. Manara, and A. Monorchio, "Analysis of Waveguide Discontinuities using Edge Elements in a Hybrid Mode Matching/Finite Elements approach," IEEE Microwave and Components Letters, vol. 11, pp. 379381, September 2001.

[31] J. Rubio, J. Arroyo, and J. Zapata, "Analysis of Passive Microwave Circuits by using a Hybrid 2D and 3D Finite Element Mode-Matching Method," IEEE Transactions on Microwave Theory and Techniques, pp. 1746-1749, September 1999.

[32] F. Arndt, R. Beyer, J. M. Reiter, T. Sievierding, and W. T., "Automated Design of Waveguide Components using Hybrid Mode-Matching/Numerical EM Building Blocks in Optimization-oriented CAD frameworks- State-of-the-art and Recent Advances," IEEE Transactions on Microwave Theory and Techniques, vol. 45, pp. 747-760, May 1997. 
[33] R. L. Ferrari, "An Extended Huygens' Principle for Modeling Scattering from General Discontinuities within Hollow Waveguides," International Journal of Numerical Modeling: Electronic Networks, Devices and Fields, vol. 14, no. 5, pp. 411-422, 2002.

[34] S. Ramo, J. R. Whinnery, and T. van Duzer, Fields and Waves in Communication Electronics. John Wiley \& Sons, Inc, 3rd ed., 1994.

[35] A. Ishimaru, Electromagnetic Wave Propagation, Radiation and Scattering. Prentice Hall, p. 172, 1991.

[36] S.-W. L. Lee, J. Boersma, C.-L. Law, and G. A. Deschamps, "Singularity in Green's Function and its Numerical Evaluation," IEEE Transactions on Antennas and Propagation, vol. 28, pp. 311-317, May 1980.

[37] P. H. Pathak, "On the Eigenfunction expansion of Electromagnetic Dyadic Green's Functions," IEEE Transactions on Antennas and Propagation, pp. 837846, 1983.

[38] C.-T. Tai, Dyadic Green Functions in Electromagnetic Theory. IEEE Press, 1994.

[39] T. Ozdemir and J. L. Volakis, "Triangular Prisms for Edge-based Vector Finite Element Analysis of Conformal Antennas," IEEE Transactions on Antennas and Propagation, vol. 45, pp. 788-797, May 1997.

[40] R. H. Hansmann, "2D Edge-based Finite Elements for Guided and Scattered Wave Problems," Master's thesis, University of Stellenbosch, 1999.

[41] J. S. Savage and A. F. Peterson, "Higher Order Vector Finite Elements for Tetrahedral Cells," IEEE Transactions on Microwave Theory and Techniques, vol. 44, pp. 874-879, June 1996.

[42] L. S. Andersen and J. L. Volakis, "Hierarchal Tangential Vector Finite Elements for Tetrahedra," IEEE Miceowave and Guided Wave Letters, vol. 8, pp. 127-129, March 1998.

[43] R. L. Ferrari and R. I. Naidu, "Finite-element modelling of high-frequency electromagnetic problems with material discontinuities," IEE Proceedings, vol. 137 Pt.A, pp. 313-320, 1990.

[44] A. H. Stroud, Numerical Quadrature and Solution of Ordinary Differential Equations. Springer-Verlag, 1974. 
[45] S. M. Rao, D. R. Wilton, and A. W. Glisson, "Electromagnetic Scattering by Surfaces of Arbitrary Shape," IEEE Transactions on Antennas and Propagation, vol. 30, pp. 409-418, May 1982.

[46] A. F. Peterson, D. R. Wilton, and R. E. Jorgenson, "Variational Nature of Galerkin and Non-Galerkin Moment Method Solutions," IEEE Transactions on Antennas and Propagation, vol. 44, pp. 500-503, April 1996.

[47] R. H. Geschke, R. L. Ferrari, D. B. Davidson, and P. Meyer, "Application of Extended Huygens' Principle to Scattering Discontinuities in Waveguide," Transactions of SA Institute of Electrical Engineers, vol. 94, pp. 28-31, July 2003.

[48] R. Sorrentino, "Planar circuits, waveguide models, and segmentation method," IEEE Transactions on Microwave Theory and Techniques, vol. 33, pp. 10571066, October 1985.

[49] M. Sundberg, P. Kildal, and T. Ohlsson, "Moment Method Analysis of a Microwave Tunnel Oven," Journal of Microwave Power and Electromagnetic Energy, vol. 33, no. 1, pp. 36-55, 1998.

[50] M. S. Tharf and G. Costache, "Finite Element Method Solutions of Field Distributions in Large Cavities," International Journal of Numerical Modelling: Electronic Networks, Devices and Fields, vol. 7, pp. 343-355, 1994.

[51] J. P. Webb and R. Miniowitz, "Analysis of 3-D Microwave Resonators using Covariant- Projection Elements," IEEE Transactions on Microwave Theory and Techniques, vol. 39, pp. 1895-1899, November 1991.

[52] B. Z. Steinberg and Y. Leviatan, "On the use of Wavelet Expansions in the Method of Moments," IEEE Transactions on Antennas and Propagation, vol. 41, pp. 610-619, May 1993.

[53] G.-W. Pan, "Orthogonal Wavelets with Applications in Electromagnetics," IEEE Transactions on Magnetics, vol. 32, pp. 975-983, May 1996.

[54] Z. Baharav and Y. Leviatan, "Impedance Matrix Compression (IMC) using Iteratively Selected Wavelet Basis," IEEE Transactions on Antennas and Propagation, vol. 46, pp. 226-233, February 1998.

[55] M. Toupikov and G.-W. Pan, "Weighted Wavelet Expansion in the Method of Moments," IEEE Transactions on Magnetics, vol. 35, pp. 1550-1553, May 1999. 
[56] T. K. Sarkar and K. Kim, "Solution of Large Dense Complex Matrix Equations utilizing Wavelet-Like Transforms," IEEE Transactions on Antennas and Propagation, vol. 47, pp. 1628-1632, October 1999.

[57] T. K. Sarkar, C. Su, R. Adve, M. Salazar-Palma, L. Garcia-Castillo, and R. R. Boix, "A Tutorial on Wavelets from an Electrical Engineering Perspective, Part 1: Discrete Wavelet Techniques," IEEE Antennas and Propagation Magazine, vol. 40, pp. 49-68, October 1998. 


\section{Appendix A}

\section{Notes for a Huygens' Code Developer}

This section is intended for anyone who would need to implement a Huygens formulation or extend the existing implementation. An outline strategy for the development is given here.

\section{A.1 Programming Tools}

Traditionally many computational electromagnetics codes are written using FORTRAN. For an experimental code, such as the one developed for this project, a very flexible programming environment, with matrix solution routines and visual output functions built in, was required. MATLAB ${ }^{\circledR}$ provided this environment. The advantage above using FORTRAN is that visual output can be obtained easily, using available builtin MATLAB ${ }^{\circledR}$ routines. Viewing three dimensional meshes, colour plots of volume magnetic fields inside the posts, were very useful tools in the developing process. However, once such an experimental code exists as a basis for future work, it is recommended that this is transferred to FORTRAN. The reason for this is that MATLAB has a severe disadvantage in that its non-vectorized commands, mainly nested loops, are performed very slowly, and it is therefore not practical for the solution of very large Huygens' problems.

\section{A.2 Mesh Generation}

Mesh generation and basis set selection are very closely linked. Apart from considering which mesh generators are available, one should also consider what geometries 


\section{Appendix A}

\section{Notes for a Huygens' Code Developer}

This section is intended for anyone who would need to implement a Huygens formulation or extend the existing implementation. An outline strategy for the development is given here.

\section{A.1 Programming Tools}

Traditionally many computational electromagnetics codes are written using FORTRAN. For an experimental code, such as the one developed for this project, a very flexible programming environment, with matrix solution routines and visual output functions built in, was required. MATLAB ${ }^{\circledR}$ provided this environment. The advantage above using FORTRAN is that visual output can be obtained easily, using available builtin MATLAB ${ }^{\circledR}$ routines. Viewing three dimensional meshes, colour plots of volume magnetic fields inside the posts, were very useful tools in the developing process. However, once such an experimental code exists as a basis for future work, it is recommended that this is transferred to FORTRAN. The reason for this is that MATLAB has a severe disadvantage in that its non-vectorized commands, mainly nested loops, are performed very slowly, and it is therefore not practical for the solution of very large Huygens' problems.

\section{A.2 Mesh Generation}

Mesh generation and basis set selection are very closely linked. Apart from considering which mesh generators are available, one should also consider what geometries 


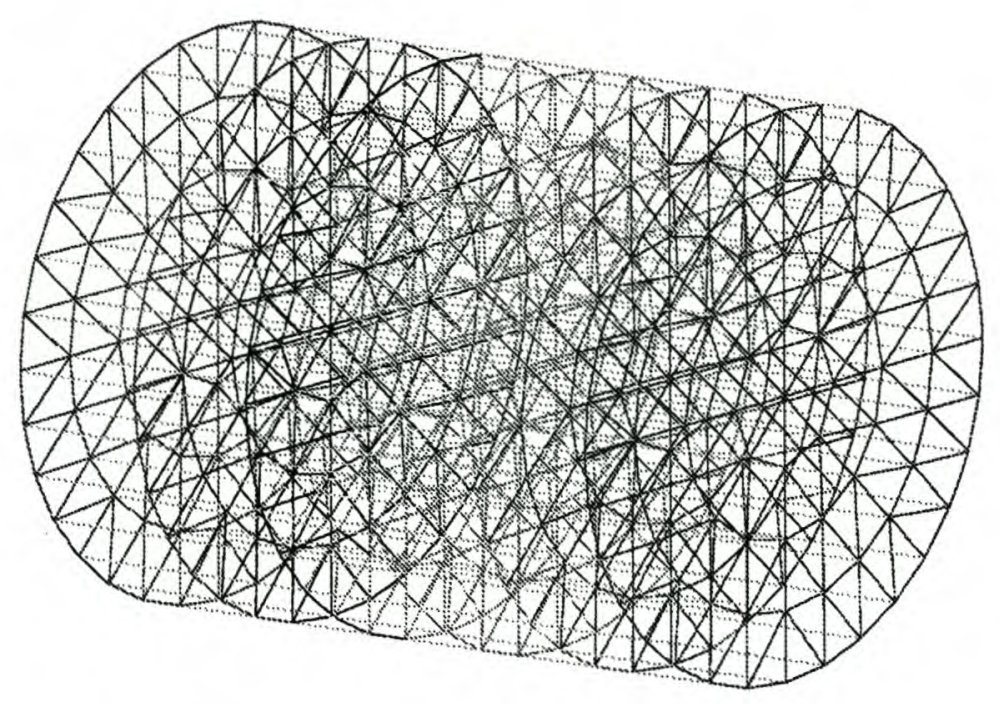

Figure A.1: $3 D$ homogeneous multi-layer prismatic mesh constructed from a $2 D$ triangular mesh

will be analyzed before making the very important choice of basis type: tetrahedral, hexagonal, prismatic or even curvilinear basis elements.

MATLAB ${ }^{\circledR}$ has a built-in 2D Delaunay mesh generator that generates a Delaunay tesselation (triangles) given a set of mesh vertices. Afterwards the edges were numbered, taking into consideration the shared edges. Boundary edges and nodes were identified and listed. This $2 \mathrm{D}$ mesh was then extruded in the y direction, providing a single layer of prismatic elements. The capability of generating homogeneous multi-layer meshes already exists in the present code: this will be required to implement more general problems that are still simple geometrically, involving both TE and TM modes, such as half-height posts. Visual output of such a mesh is shown in figure A.1.

Finally, for completely arbitrary obstacles, a tetrahedral discretization is strongly recommended. Good tetrahedral mesh generators are available commercially. Furthermore, higher order basis sets are already defined and tested, while these elemental matrices are readily available in the literature. One notes that the basis sets used here have one drawback: the prismatic basis set is not entirely coordinate independent, which is non-ideal from the elemental matrix construction point of view. 


\section{A.3 Developing Strategy: A Modular Approach}

The entire code may be divided into sections, some of which may be tested and debugged separately before combining them. A suggestion follows below for such an approach.

- Firstly, the finite element discretization must be considered, using either a commercial mesh generator or a simple self-developed generator. To test the $[S]$ and $[T]$ matrix separately, a finite element discretization of a section of empty $\mathrm{X}$-band waveguide in a travelling wave configuration was used to run a number of convergence tests with successive mesh refinement. This procedure was described in section 3.4. Now confidence has been established in the [S] and [T] matrices including the mesh and edge numbering integrity.

- The connection between the volume and surface fields through the $\left[B_{E}\right]$ matrix is examined by the procedure described in section 3.5. This establishes confidence in the connection between the MoM surface integrals and the FE discretization.

- Not all parts of the MoM surface discretization may be examined separately; however, considering a PEC post, the finite element mesh reduces to a surface mesh, enabling the surface integrations of $\left[M_{E}\right]$ and the excitation $\left\{e^{ \pm n}\right\}$ to be tested without the finite element magnetic field variables. Considering a PMC post, the surface integrations of $\left[M_{H}\right]$ and the excitation $\left\{e^{ \pm n}\right\}$ can be tested along with the connection through $\left[B_{E}\right]$.

- Finally all the components may be combined. The finite element and surface numbering here must be consistent. Some edges are directional and the order of node numbering is important. At this stage careful tests to ensure convergence of the numerical integration, the modal sums and the finite element discretization are required. 


\section{Appendix B}

\section{Derivation of Tangential Basis Function Component}

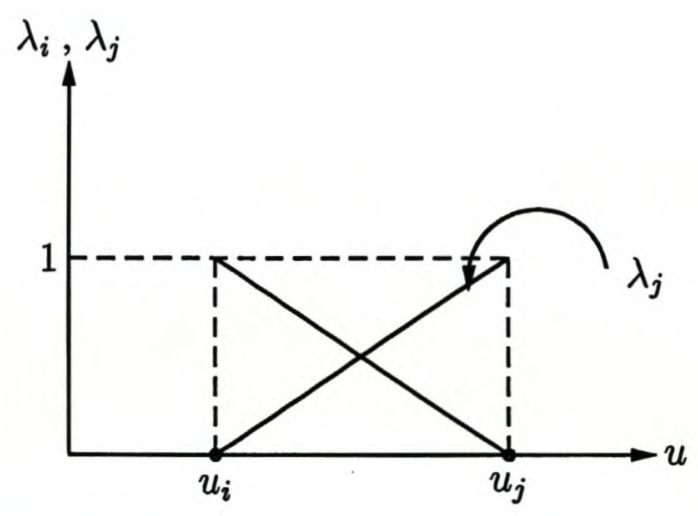

Figure B.1: The two non-zero simplex coordinates for a patch element.

Consider a single rectangular patch element. Since the y integration is performed analytically and the basis functions have no $y$ variation, only the $u$ axis is shown here. The element boundaries on the $u$ axis are at $u=u_{i}$ and $u=u_{j}$ as shown in figure B.1. Integration is from $u_{i}$ to $u_{j}$ and in a consistent anticlockwise direction around the post. The variation of the two simplex coordinates defined on this axis is also indicated. Note that the third triangular simplex coordinate is zero on this axis. Let $d_{i}=u_{j}-u_{i}$. From inspection of figure B.1, we find expressions for the simplex coordinates in terms of the coordinate system used here:

$$
\begin{aligned}
& \lambda_{j}=\frac{1}{u_{j}-u_{i}} u-\frac{u_{i}}{u_{j}-u_{i}} \\
& \lambda_{i}=\frac{-1}{u_{j}-u_{i}} u+\frac{u_{j}}{u_{j}-u_{i}}
\end{aligned}
$$


To perform the surface integrations, the tangential surface contribution of each basis function should be determined. For both basis sets the contribution is a constant or a linear function expressed in terms of the $B_{u}$, and $B_{k}$ coefficients of sections 3.9 and 3.10 .

\section{B.1 $\hat{\mathbf{n}} \times \mathbf{W}_{l}$}

This basis function is $u$-directed. It is well known that the tangential component of $\mathbf{W}_{l}$ to an edge is a constant [21].

Using the relationships $\hat{\mathbf{u}} \cdot \nabla \lambda_{j}=\frac{1}{d_{i}}$ and $\hat{\mathbf{u}} \cdot \nabla \lambda_{i}=-\frac{1}{d_{i}}([21])$, we find that $\hat{\mathbf{u}} \cdot \hat{\mathbf{n}} \times \mathbf{W}_{l}=$ 1. Thus we have $B_{u}=0$ and $B_{k}=1$.

\section{B.2 $\hat{\mathbf{n}} \times \mathrm{M}_{l}$}

This basis function is $u$-directed and linear in $u$. In this case we have $\hat{\mathbf{u}} \cdot \hat{\mathbf{n}} \times \mathbf{M}_{l}=$ $\lambda_{i}-\lambda_{j}$. Using the expressions for $\lambda_{i}$ and $\lambda_{j}$,

$$
B_{u}=\frac{2}{u_{j}-u_{i}}
$$

and

$$
B_{k}=\frac{u_{i}+u_{j}}{u_{j}-u_{i}}
$$

\section{B.3 $\hat{\mathbf{n}} \times \mathbf{F}_{l}$}

The so called face basis functions have no tangential contribution on any face, as $\nabla \lambda_{i}$ is perpendicular to one edge while on the edges, either $\lambda_{i}$ or $\lambda_{j}$ is zero.

\section{B.4 $\hat{\mathbf{n}} \times \mathbf{K}_{l}$}

The basis function tangential components are y-directed and are linear functions with 
Chapter B - DeRIVATION of TANgential Basis Function Component VI

$$
\begin{aligned}
& B_{u}= \begin{cases}\frac{1}{u_{j}-u_{i}} & : \mathbf{K}_{l}=\lambda_{i} \hat{\mathbf{y}} \\
-\frac{1}{u_{j}-u_{i}} & : \mathbf{K}_{l}=\lambda_{i} \hat{\mathbf{y}}\end{cases} \\
& B_{k}=\left\{\begin{array}{cl}
-\frac{u_{i}}{u_{j}-u_{i}} & : \mathbf{K}_{l}=\lambda_{i} \hat{\mathbf{Y}} \\
\frac{u_{j}}{u_{j}-u_{i}} & : \mathbf{K}_{l}=\lambda_{i} \hat{\mathbf{y}}
\end{array}\right.
\end{aligned}
$$




\section{Appendix C}

\section{CT/LN versus LT/QN basis sets}

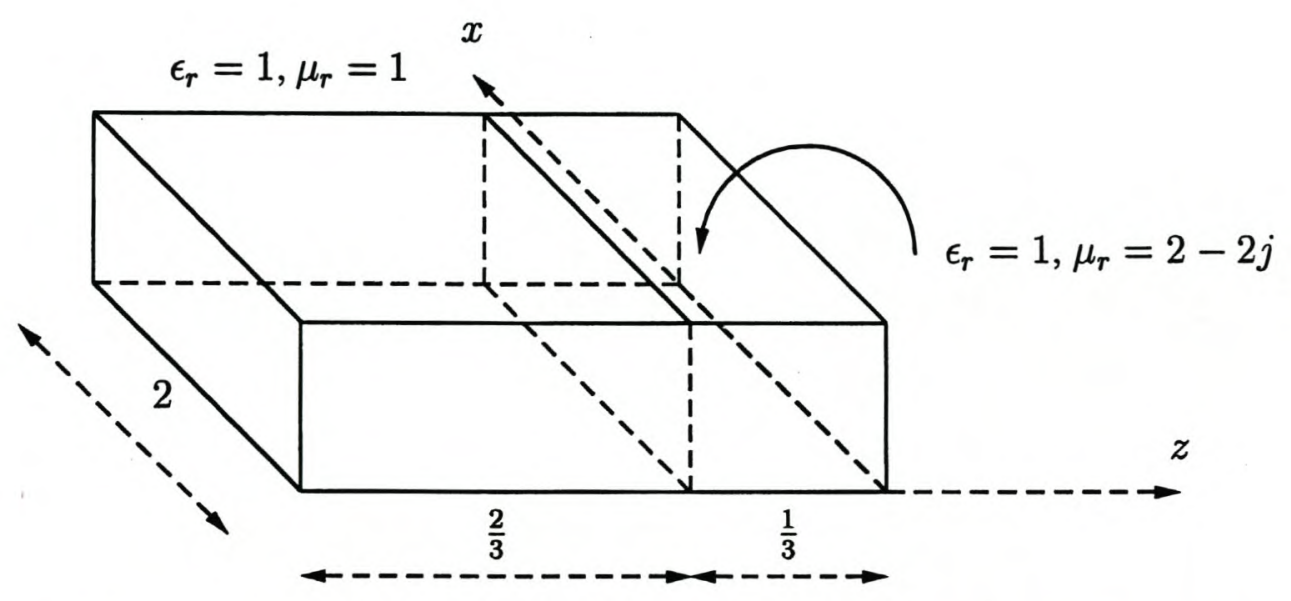

Figure C.1: Geometry of a test problem with material discontinuities, repeated here for convenience

The validity of the LT/QN basis set matrix entries are demonstrated here by considering the same geometry and problem setup as in section 3.6. The result of figure 3.7 for the 170 element CT/LN discretization (440 degrees of freedom), compared here with a 170 element LT/QN discretization (990 degrees of freedom), is shown here in figure C.2. 


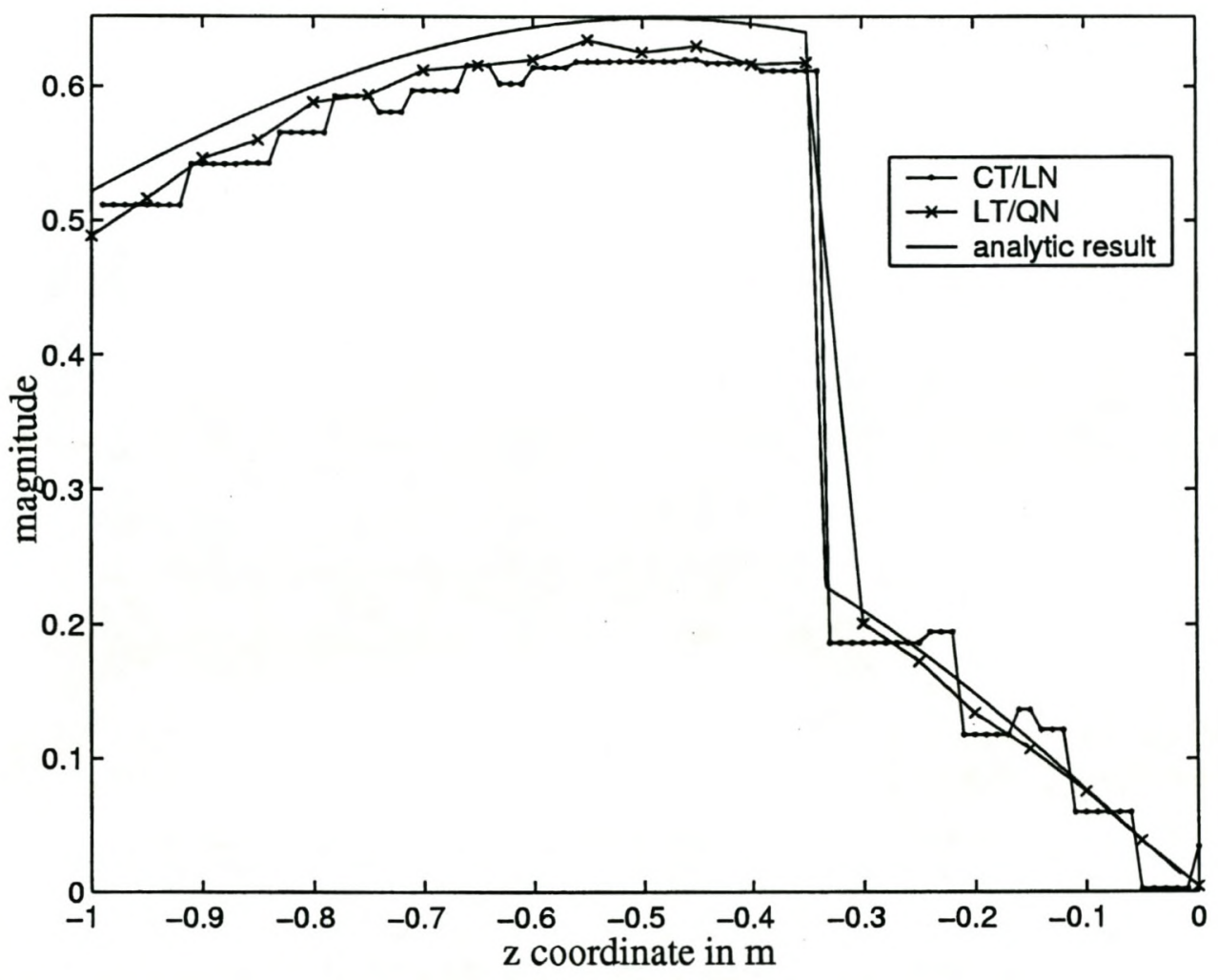

Figure C.2: Magnitude of $H_{z}$ field at $x=0$, using the connection matrix and Finite Element solution, compared to an analytic solution. The waveguide was discretized into 170 elements for both the CT/LN and LT/QN case. 